\title{
ESPACES SYMÉTRIQUES DE DRINFELD ET CORRESPONDANCE DE LANGLANDS LOCALE
}

\author{
PAR JEAN FRANÇOIS DAT
}

RÉSUMÉ. - Nous étudions les complexes de cohomologie équivariants des espaces symétriques $p$-adiques de Drinfeld munis de leur action de Galois, et nous montrons comment ils permettent de réaliser la correspondance de Langlands locale pour certaines représentations de $G L_{d}$ dites «principales elliptiques ». Il s'agit de la première étape d'une généralisation conjecturale de la théorie de Lubin-Tate non-abélienne de Carayol, qui fournirait une réalisation cohomologique de la correspondance de Langlands pour toutes les représentations elliptiques. Au cours de notre étude, nous calculons tous les groupes d'extensions entre représentations elliptiques et les cup-produits correspondants, nous donnons un nouveau calcul de la cohomologie à supports compacts des espaces symétriques $p$-adiques, et nous obtenons une nouvelle preuve de la conjecture monodromie-poids de Deligne pour les variétés uniformisées par ces espaces.

(c) 2006 Elsevier SAS

ABSTRACT. - We study the Galois action on the equivariant cohomology complex of Drinfeld's $p$-adic symmetric spaces and show how it encodes Langlands' correspondence for the so-called "principal elliptic" representations of $G L_{d}$. This is the first stage of an expected generalization of Carayol's non-Abelian LubinTate theory from supercuspidal to elliptic representations. In the process we obtain a new proof of Deligne's weight-monodromy conjecture for those varieties which admit $p$-adic uniformization by these spaces, we compute Ext groups and cup-products for elliptic representations, and we give a new computation of the compactly supported cohomology of $p$-adic symmetric spaces.

(c) 2006 Elsevier SAS

\section{Introduction et principaux résultats}

Soit $K$ un corps local non-archimédien de caractéristique résiduelle $p$, dont on fixe une clôture algébrique $K^{\text {ca }}$ de complété $\widehat{K^{\mathrm{ca}}}$. Pour un entier $d>1$, le «demi-plan supérieur» ou «espace symétrique » de Drinfeld est défini dans [17] comme un sous- $K$-espace analytique rigide de l'espace projectif $\mathbb{P}^{d-1}$. Ses $\widehat{K^{\mathrm{ca}}}$-points sont donnés par

$$
\Omega_{K}^{d-1}\left(\widehat{K^{\mathrm{ca}}}\right)=\mathbb{P}^{d-1}\left(\widehat{K^{\mathrm{ca}}}\right) \backslash \bigcup_{\mathbb{H} \in \mathcal{H}_{K}} \mathbb{H}\left(\widehat{K^{\mathrm{ca}}}\right)
$$

où $\mathcal{H}_{K}$ désigne l'ensemble des hyperplans $K$-rationnels de $\mathbb{P}^{d-1}$. Nous désignerons par le même symbole $\Omega_{K}^{d-1}$ le $K$-espace analytique de Berkovich correspondant dont on trouve une description dans [4]; ses points s'identifient à des classes d'équivalence de semi-normes multiplicatives sur l'anneau de polynômes $K\left[X_{0}, \ldots, X_{d-1}\right]$ dont la restriction au sous-espace des polynômes homogènes de degré 1 est une norme. 
L'espace analytique $\Omega_{K}^{d-1}$ est naturellement muni d'une action de $G L_{d}(K)$ triviale sur le centre, qui est continue au sens de [3, par. 6-7]. Le changement de base $\Omega_{K}^{d-1, \text { ca }}:=\Omega_{K}^{d-1} \widehat{\otimes}_{K} \widehat{K^{\mathrm{ca}}}$ est aussi muni d'une action continue du groupe de Weil $W_{K}$ de $K$ (et même de son groupe de Galois). On s'intéresse à la cohomologie étale à supports compacts de $\Omega_{K}^{d-1}$, munie de son action du groupe $G L_{d}(K) \times W_{K}$. Par des résultats généraux de Berkovich, on sait que l'action de $G L_{d}(K)$ sur ces groupes de cohomologie est lisse. Le formalisme de Berkovich permet aussi, lorsque $\Lambda$ est un anneau de torsion première à $p$, de définir un complexe naturel

$$
R \Gamma_{c}\left(\Omega_{K}^{d-1, \mathrm{ca}}, \Lambda\right) \in D_{\Lambda}^{b}\left(P G L_{d}(K)\right)
$$

dans la catégorie dérivée des $\Lambda P G L_{d}(K)$-modules lisses, et dont les objets de cohomologie sont les $H_{c}^{i}\left(\Omega_{K}^{d-1, \mathrm{ca}}, \Lambda\right)$. Nous expliquons sa construction et nous l'adaptons aux coefficients $l$-adiques dans l'appendice B.

Supposons momentanément que $\Lambda=\overline{\mathbb{Q}}_{l}$ et appelons « séries principales elliptiques » les sousquotients irréductibles de la représentation régulière de $G L_{d}(K)$ dans l'espace $\overline{\mathbb{Q}}_{l}^{\infty}\left[\mathcal{B}_{d}(K)\right]$ des fonctions localement constantes à valeurs dans $\overline{\mathbb{Q}}_{l}$ sur les points rationnels de la variété des drapeaux $\mathcal{B}_{d}$ de $G L_{d}$. La correspondance de Langlands locale $[31,22,24]$ associe à une représentation lisse irréductible $\pi$ de $G L_{d}(K)$ une représentation continue $\sigma_{d}(\pi)$ de $W_{K}$ dans un $\overline{\mathbb{Q}}_{l}$-espace de dimension $d$. Dans le cas des séries principales elliptiques, la représentation $\sigma_{d}(\pi)$ est bien sûr connue depuis longtemps. Un des résultats principaux de ce texte est le suivant :

1.1. ThÉORÈme. - Pour toute série principale elliptique $\pi$ de $G L_{d}(K)$, il existe un isomorphisme $W_{K}$-équivariant

$$
\mathcal{H}^{*}\left(R \operatorname{Hom}_{D_{\overline{\mathbb{Q}}_{l}}\left(P G L_{d}(K)\right)}\left(R \Gamma_{c}\left(\Omega_{K}^{d-1, \mathrm{ca}}, \overline{\mathbb{Q}}_{l}\right), \pi\right)\right) \stackrel{\sim}{\longrightarrow} \sigma_{d}(\pi) \otimes|-|^{\frac{d-1}{2}} .
$$

Si $\pi$ est lisse irréductible mais pas principale elliptique alors le terme de gauche est nul.

Expliquons nos notations et le sens de l'énoncé; le complexe $R \Gamma_{c}\left(\Omega_{K}^{d-1, \text { ca }}, \overline{\mathbb{Q}}_{l}\right)$ est muni d'une action de $W_{K}$, de sorte que pour toute représentation lisse $\pi$ de $G L_{d}(K)$, le complexe de $\overline{\mathbb{Q}}_{l}$-espaces vectoriels

$$
R \operatorname{Hom}_{D_{\overline{\mathbb{Q}}_{l}}\left(P G L_{d}(K)\right)}\left(R \Gamma_{c}\left(\Omega_{K}^{d-1, \mathrm{ca}}, \overline{\mathbb{Q}}_{l}\right), \pi\right) \in D^{b}\left(\overline{\mathbb{Q}}_{l}\right)
$$

est aussi muni d'une action de $W_{K}$. Rappelons que la catégorie triangulée $D^{b}\left(\overline{\mathbb{Q}}_{l}\right)$ est équivalente à la catégorie triangulée des $\overline{\mathbb{Q}}_{l}$-espaces vectoriels $\mathbb{Z}$-gradués «à support fini », une équivalence étant donnée par le foncteur $\mathcal{H}^{*}: \mathcal{C}^{\bullet} \in D^{b}\left(\overline{\mathbb{Q}}_{l}\right) \mapsto \bigoplus_{i \in \mathbb{Z}} \mathcal{H}^{i}\left(\mathcal{C}^{\bullet}\right)$. Ainsi le $\overline{\mathbb{Q}}_{l}$-espace vectoriel $\mathbb{Z}$-gradué

$$
\mathcal{H}^{*}\left(R \operatorname{Hom}_{D_{\overline{\mathbb{Q}}_{l}}\left(P G L_{d}(K)\right)}\left(R \Gamma_{c}\left(\Omega_{K}^{d-1, \mathrm{ca}}, \overline{\mathbb{Q}}_{l}\right), \pi\right)\right)
$$

est muni d'une action de $W_{K}$. Dans l'énoncé du théorème, on oublie la $\mathbb{Z}$-graduation pour se retrouver avec des représentations au sens usuel. Enfin, la notation $|-|$ désigne le caractère non-ramifié de $W_{K}$ qui envoie les Frobenius arithmétiques sur l'ordre $q$ du corps résiduel de $K$.

Expliquons maintenant ce que l'énoncé du théorème peut avoir de surprenant; dans [38], Schneider et Stuhler ont calculé les groupes de cohomologie (sans supports) de $\Omega_{K}^{d-1}$, munis de leur action (pas lisse) de $G L_{d}(K)$, pour toute théorie cohomologique satisfaisant certains axiomes. La cohomologie étale à supports compacts ne satisfait pas ces axiomes, mais il est facile de deviner que les $H_{c}^{i}$ se déduisent des $H^{2 d-2-i}$ par contragrédiente lisse, ce que nous 
vérifierons dans la partie 3. Ces $H_{c}^{i}$ se trouvent être des séries principales elliptiques lorsqu'ils sont non nuls, i.e. lorsque $i=d-1, \ldots, 2 d-2$. Ainsi, sur les $2^{d-1}$ séries principales elliptiques seulement $d$ apparaissent dans la cohomologie, mais le théorème dit en substance que toutes sont détectées par le complexe de cohomologie. Quant à l'action de $W_{K}$ sur $H_{c}^{d-1+i}$, elle est on ne peut plus simple puisqu'elle se fait via le caractère $|-|^{-i}$. En particulier, l'action de l'inertie $I_{K}$ est triviale en cohomologie. Or, les représentations $\sigma_{d}(\pi)$ pour $\pi$ principale elliptique, sont essentiellement déterminées par leur restriction à $I_{K}$, elle-même décrite par l'opérateur nilpotent $N$ associé à la partie unipotente de la monodromie. Le théorème dit en substance que l'action de $W_{K}$ sur le complexe de cohomologie est suffisamment non triviale pour pouvoir récupérer les $\sigma_{d}(\pi)$.

Le principe de la preuve du théorème 1.1 n'est pas subtil : on décrit explicitement le complexe $R \Gamma_{c}$ muni de son action de $W_{K}$. Il s'avère que l'action de l'inertie est unipotente donnée par un opérateur $N$ dont il faut-entre autres-déterminer l'ordre de nilpotence. En résolvant cette question nous avons été amené à donner une nouvelle preuve du résultat suivant dû à Itô :

1.2. ThÉORÈmE. - Les variétés algébriques sur $K$ uniformisées par $\Omega_{K}^{d-1}$ satisfont la conjecture de Deligne sur la pureté de la filtration de monodromie (conjecture MonodromiePoids).

Nous renvoyons à 4.5.1 pour un énoncé plus détaillé. Bien que nous utilisions la suite spectrale de Rapoport-Zink pour les schémas semi-stables, notre preuve est vraiment de nature «théorie des groupes » contrairement à celle d'Itô qui est plus géométrique. Nous obtenons aussi la semisimplicité des relèvements de Frobenius.

L'ingrédient essentiel de la preuve des théorèmes précédents est le calcul explicite des extensions entre séries principales elliptiques et de leurs $U$-produits. Celui-ci a un sens sur un groupe réductif $p$-adique $G$ quelconque et est effectué dans la partie 2 dans le cas déployé. Soit $B$ un sous-groupe de Borel de $G$, on utilise la paramétrisation des sous-quotients irréductibles de $\mathcal{C}_{\mathbb{Q}_{l}}^{\infty}(G / B)$ par les sous-ensembles de l'ensemble $S$ des racines simples d'un tore maximal $T$ de $B$ dans $\operatorname{Lie}(B)$ donnée par

$$
(I \subseteq S) \mapsto \pi_{I}:=\mathcal{C}_{\overline{\mathbb{Q}}_{l}}^{\infty}\left(G / P_{I}\right) / \sum_{J \supset I} \mathcal{C}_{\overline{\mathbb{Q}}_{l}}^{\infty}\left(G / P_{J}\right)
$$

où $P_{I}$ est le parabolique contenant $B$ associé à $I$ (cf. 2.1.3). Voici l'énoncé principal de la partie 2:

1.3. ThÉORÈME. - Supposons $G$ semi-simple et posons $\delta(I, J)=|I \cup J|-|I \cap J|$ pour $I, J \subseteq S$.

(i) Soient I, J deux sous-ensembles de $S$, alors:

$$
\operatorname{Ext}_{G}^{*}\left(\pi_{I}, \pi_{J}\right)= \begin{cases}\overline{\mathbb{Q}}_{l} & \text { si } *=\delta(I, J), \\ 0 & \text { si } * \neq \delta(I, J) .\end{cases}
$$

(ii) Soient $I, J, K$ trois sous-ensembles de $S$ tels que $\delta(I, J)+\delta(J, K)=\delta(I, K)$; alors le cup-produit

$$
\cup: \operatorname{Ext}_{G}^{\delta(I, J)}\left(\pi_{I}, \pi_{J}\right) \otimes_{\overline{\mathbb{Q}}_{l}} \operatorname{Ext}_{G}^{\delta(J, K)}\left(\pi_{J}, \pi_{K}\right) \rightarrow \operatorname{Ext}_{G}^{\delta(I, K)}\left(\pi_{I}, \pi_{K}\right)
$$

est un isomorphisme. 
Signalons que le point (i) a aussi été obtenu indépendamment par Orlik dans [34], par une méthode plus directe et moins explicite que la nôtre. Certains cas particuliers importants de ce point (i) avaient été préalablement obtenus par Borel, Casselman et Schneider-Stuhler.

Dans le corps du texte, nous avons énoncé la plupart des résultats pour des anneaux de coefficients plus généraux que $\overline{\mathbb{Q}}_{l}$, voir les théorèmes 2.1 .4 et 4.1 .5 . Par exemple pour $\Lambda$ un corps de caractéristique positive «fortement banale», 4.1.5 donne un analogue de 1.1 pour la correspondance de Langlands-Vignéras. Ceci complique un peu certains arguments de théorie des représentations, et mêmes certains arguments géométriques : nous avons par exemple été amené à vérifier que le formalisme des cycles évanescents de Rapoport-Zink s'applique encore à certains schémas formels «fortement semi-stables».

Venons-en maintenant à la généralisation que nous espérons obtenir de ces résultats dans un travail ultérieur. Soit $D_{d}^{\times}$le groupe des inversibles de l'algèbre à division d'invariant $1 / d$ sur $K$. Drinfeld a attaché à toute représentation irréductible $\rho$ de $D_{d}^{\times}$, de caractère central $\omega_{\rho}: K^{\times} \rightarrow \overline{\mathbb{Q}}_{l}$ d'ordre fini, un $\overline{\mathbb{Q}}_{l}$-système local $G L_{d}(K)$-équivariant $\mathcal{L}_{\rho}$ sur $\Omega_{K}^{d-1}$. On peut à nouveau lui associer un complexe $R \Gamma_{c}\left(\Omega_{K}^{d-1, \text { ca }}, \mathcal{L}_{\rho}\right)$ dans la catégorie dérivée $D_{\omega_{\rho}}^{b}\left(G L_{d}(K)\right)$ des représentations de caractère central $\omega_{\rho}$. On dit qu'une représentation lisse irréductible de $G L_{d}(K)$ est «elliptique de type $\rho$ » si elle a même caractère infinitésimal que la représentation $J L_{d}(\rho)$ associée à $\rho$ par correspondance de Jacquet-Langlands.

1.3.1. Conjecture. - Pour toute série principale elliptique $\pi$ de type $\rho$ de $G L_{d}(K)$, il existe un isomorphisme $W_{K}$-équivariant

$$
\mathcal{H}^{*}\left(R \operatorname{Hom}_{D_{\omega_{\rho}}^{b}\left(G L_{d}(K)\right)}\left(R \Gamma_{c}\left(\Omega_{K}^{d-1, \mathrm{ca}}, \mathcal{L}_{\rho}\right), \pi\right)\right) \stackrel{\sim}{\longrightarrow} \sigma_{d}(\pi) \otimes|-|^{\frac{d-1}{2}} .
$$

Si $\pi$ est lisse irréductible mais pas «elliptique de type $\rho$ », alors le terme de gauche est nul.

Cet énoncé généralise celui de Carayol dans [13] et serait en quelque sorte l'aboutissement de sa «théorie de Lubin-Tate non-abélienne», au moins du côté des espaces symétriques de Drinfeld. Notons qu'il contient en lui la conjecture Monodromie-Poids (et la semi-simplicité des Frobenius) pour les variétés uniformisées par les revêtements de Drinfeld de $\Omega_{K}^{d-1}$. Les méthodes purement locales et géométriques du présent texte (qui traite le cas $\rho=\overline{\mathbb{Q}}_{l}$ ), seront bien insuffisantes pour le cas général. Néanmoins nous espérons dans un travail ultérieur déduire ce cas général d'un travail en cours de Boyer sur la cohomologie de la tour de Lubin-Tate (où les outils sont fondamentalement de nature globale), via les méthodes de Faltings dans [18], actuellement remaniées et généralisées par Fargues, Genestier et V. Lafforgue (qui, elles, sont de nature purement locales).

Organisation de l'article : Le cœur de ce texte est la partie 4 ; on y prouve les théorèmes 1.1 et 1.2 ci-dessus. Il est conseillé de la lire directement après avoir lu les énoncés et notations des théorèmes 2.1.4 et 3.1.1. La partie 2 est consacrée à la preuve du théorème 1.3. La partie 3 contient le calcul de la cohomologie à supports compacts de $\Omega_{K}^{d-1}$ : comme nous n'avons pas su adapter la stratégie de Schneider et Stuhler aux coefficients $l$-adiques, notre calcul suit une autre méthode que Drinfeld avait utilisée en rang $d=2$. Le premier appendice contient quelques lemmes généraux sur les $t$-catégories utilisés dans la partie 4. Le second fournit les fondements nécessaires pour la partie 4 ; nous exposons le formalisme de cohomologie continue de torsion de Berkovich, puis nous en proposons une extension aux coefficients $l$-adiques et démontrons une compatibilité aux quotients. Le point de vue que nous adoptons est différent de celui de Harris dans [20,21], et plutôt inspiré de celui de Fargues [19].

$4^{e}$ SÉRIE - TOME $39-2006-\mathrm{N}^{\circ} 1$ 


\section{Extensions dans la série principale}

\subsection{Notations et premier énoncé}

2.1.1. Soit $\mathbf{G}$ un groupe réductif déployé sur un corps local non-archimédien $F$ dont on fixe un tore maximal déployé $\mathbf{T}$ et un sous-groupe de Borel $\mathbf{B}_{+}$de radical unipotent $\mathbf{U}_{+}$. On notera les groupes de points $F$-rationnels par les caractères non épaissis correspondants $G, T, B_{+}$. En désignant par $X_{*}(\mathbf{T})$ le groupe des cocaractères de $\mathbf{T}$, on pose $X:=X_{*}(\mathbf{T}) \otimes \mathbb{R}$. L'ensemble $S$ des racines simples de $\mathbf{T}$ dans $\operatorname{Lie}\left(\mathbf{U}_{+}\right)$sera parfois considéré comme un sous-ensemble du dual $X^{*}$ de $X$. Le groupe de Weyl correspondant est noté $W$. Comme d'habitude, un sous-groupe parabolique sera dit standard s'il contient $\mathbf{B}_{+}$et un sous-groupe de Levi sera dit standard si c'est la composante de Levi contenant $\mathbf{T}$ d'un sous-groupe parabolique standard. Les sous-groupes de Levi standards sont en bijection avec les sous-ensembles de $S$.

2.1.2. Pour $J \subseteq S$, on notera $\mathbf{M}_{J}$ le sous-groupe de Levi standard dont le système de racines de $\mathbf{T}$ associé est engendré par $J$. On a donc $\mathbf{M}_{J}=\mathcal{Z}_{\mathbf{G}}\left(\bigcap_{\alpha \in J} \operatorname{ker} \alpha\right)^{0}$. On notera aussi $\mathbf{A}_{J}$ le centre connexe de $\mathbf{M}_{J}$ et $a_{J}:=X_{*}\left(\mathbf{A}_{J}\right) \otimes \mathbb{R}$. On remarquera que $a_{\emptyset}=X$. En général, l'injection canonique $X_{*}\left(\mathbf{A}_{J}\right) \rightarrow X_{*}(\mathbf{T})$ identifie $a_{J}$ à

$$
\{x \in X, \forall \alpha \in J,\langle x, \alpha\rangle=0\} .
$$

\subsubsection{Représentations}

Si $R$ est un anneau commutatif tel que $p \in R^{\times}$, on dit qu'un $R$-module $V$ muni d'une action $\pi$ de $G$ est lisse si le stabilisateur de tout élément est ouvert; on dit de plus qu'un tel $R G$ module est admissible si pour tout sous-groupe ouvert compact $H$, le $R$-module $V^{H}$ des $H$ invariants est de type fini. On omettra souvent le $V$ de nos notations en désignant par $\pi$ à la fois la représentation de $G$ et son $R$-module sous-jacent. La catégorie abélienne des $R G$-modules lisses sera notée $\operatorname{Mod}_{R}(G)$. Cette catégorie a suffisamment d'objets projectifs et injectifs. Nous noterons simplement $\operatorname{Ext}_{R G}^{*}(-,-)$ les groupes d'extensions dans cette catégorie.

Soit $J \subset S$. Notons $P_{J}$ le groupe parabolique standard engendré par $M_{J}$ et $B_{+}$. Pour tout $K \supset J$, on a une injection canonique $\mathcal{C}_{R}^{\infty}\left(G / P_{K}\right) \hookrightarrow \mathcal{C}_{R}^{\infty}\left(G / P_{J}\right)$. On pose alors

$$
\pi_{J}^{R}:=\mathcal{C}_{R}^{\infty}\left(G / P_{J}\right) /\left(\sum_{K \supset J} \mathcal{C}_{R}^{\infty}\left(G / P_{K}\right)\right) .
$$

C'est une $R$-représentation admissible de $G$. Notre but ici est de démontrer le théorème suivant, dans lequel on utilise la notation $\delta(I, J)$ pour le cardinal de la différence symétrique $\Delta(I, J):=$ $(I \cup J) \backslash(I \cap J)$ entre deux sous-ensembles $I, J$ de $S$.

2.1.4. ThÉORÈME. - Supposons $\mathbf{G}$ semi-simple et soit $R$ un anneau fortement banal pour $G$ au sens de 2.1.6 ci-dessous.

(i) Soient I, J deux sous-ensembles de $S$, alors:

$$
\operatorname{Ext}_{R G}^{*}\left(\pi_{I}^{R}, \pi_{J}^{R}\right)= \begin{cases}R & \text { si } *=\delta(I, J) \\ 0 & \text { si } * \neq \delta(I, J)\end{cases}
$$

(ii) Soient $I, J, K$ trois sous-ensembles de $S$ tels que $\delta(I, J)+\delta(J, K)=\delta(I, K)$, alors le cup-produit

$$
\cup: \operatorname{Ext}_{R G}^{\delta(I, J)}\left(\pi_{I}^{R}, \pi_{J}^{R}\right) \otimes_{R} \operatorname{Ext}_{R G}^{\delta(J, K)}\left(\pi_{J}^{R}, \pi_{K}^{R}\right) \rightarrow \operatorname{Ext}_{R G}^{\delta(I, K)}\left(\pi_{I}^{R}, \pi_{K}^{R}\right)
$$

est un isomorphisme. 
Ce théorème sera précisé par la proposition 2.2.11 dans laquelle nous construisons de manière explicite des extensions de Yoneda engendrant les Ext non-triviaux qui sont décrits ci-dessus. Il généralise des résultats précédents de Casselman et Schneider-Stuhler [38, Prop. 5.9]. Par ailleurs le point (i) a aussi été obtenu indépendamment par S. Orlik via une méthode plus directe mais moins explicite, sous l'hypothèse supplémentaire que $R$ est auto-injectif, mais pour des groupes pas nécessairement déployés.

\subsubsection{Bonnes caractéristiques}

Suivant une terminologie introduite par Vignéras, on dira qu'un nombre premier $l$ est banal pour $G$ s'il ne divise pas l'ordre d'un sous-groupe compact de $G$. On notera aussi

$$
N_{G}:=\left(p^{\prime} \text {-partie du pro-ordre de } G\right) \in \mathbb{N}^{\times} .
$$

Lorsqu'on considère des représentations de $G$ à valeurs dans un corps, l'hypothèse «de caractéristique banale» nous permettra d'utiliser des résultats de finitude cohomologique et de fidélité des foncteurs de Jacquet sur les représentations de la série principale. Mais pour des raisons de calculs d'exposants (cf. surtout le lemme 2.3.11), cette hypothèse ne suffira pas pour nos arguments.

Notons $\rho \in X^{*}(\mathbf{T})$ le déterminant de l'action de $\mathbf{T}$ sur Lie $\mathbf{U}_{+}$. Vu comme élément de $X^{*}$, c'est aussi la somme des racines $S$-positives et on peut écrire $\rho=\sum_{\alpha \in S} n_{\alpha} \alpha$ pour des entiers positifs $n_{\alpha}$. On dira qu'un nombre premier $l$ est bon pour $G$ si le cardinal $q_{F}$ du corps résiduel $k_{F}$ de $F$ est d'ordre $\geqslant \sup _{\alpha}\left(n_{\alpha}\right)$ modulo $l$, ou ce qui est équivalent si $l$ est premier à l'entier

$$
N_{W}:=\prod_{k \leqslant \sup \left(n_{\alpha}\right)}\left(q_{F}^{k}-1\right) .
$$

Pour $G L(n)$, on a $(n-1)(n+1) / 4 \leqslant \sup \left(n_{\alpha}\right) \leqslant n^{2} / 4$, et on voit donc que pour $n \geqslant 4$, «bon» implique «banal». Cependant, en petit rang, il peut exister des $l$ non banals et bons au sens ci-dessus, par exemple pour $G L(2)$.

2.1.6. DÉfinition. - Nous dirons dorénavant qu'un anneau $R$ est banal, resp. fortement banal pour $G$ si l'entier $p N_{G}$, resp. l'entier $p N_{G} N_{W}$ est inversible dans $R$.

Nous n'utilisons dans ce texte que le calcul pour un groupe semi-simple (et même pour $\mathbf{P G L}_{\mathbf{n}}$ ). Mentionnons tout de même le calcul pour un groupe réductif (déployé).

2.1.7. Corollaire. - Supposons $\mathbf{G}$ réductif déployé, de centre de dimension d, et $R$ fortement banal pour $G$. Alors pour tous sous-ensembles $I, J$ de $S$, on a

$$
\operatorname{Ext}_{G}^{*}\left(\pi_{I}^{R}, \pi_{J}^{R}\right)= \begin{cases}R^{\left(\begin{array}{l}
d \\
r
\end{array}\right)} & \text { si } *=\delta(J, I)+r, 0 \leqslant r \leqslant d, \\
0 & \text { si } *<\delta(J, I) \text { ou } *>\delta(J, I)+d .\end{cases}
$$

Preuve. - (À partir du cas semi-simple.) Notons $Z$ le centre de $G$. Le foncteur d'inflation $\operatorname{Mod}_{R}(G / Z) \rightarrow \operatorname{Mod}_{R}(G)$ est exact et admet pour adjoint à gauche le foncteur des $Z$-coinvariants

$$
\begin{aligned}
\operatorname{Mod}_{R}(G) & \rightarrow \operatorname{Mod}_{R}(G / Z), \\
V & \mapsto V_{Z}:=V /\langle v-z v, v \in V, z \in Z\rangle .
\end{aligned}
$$

Ce dernier envoie donc projectifs sur projectifs, et est exact à droite. On a donc la composée de foncteurs dérivés suivante

$$
R \operatorname{Hom}_{G}\left(?, \pi_{I}^{R}\right) \simeq R \operatorname{Hom}_{G / Z}\left(L\left(? \mapsto ?_{Z}\right), \pi_{I}^{R}\right) .
$$

$4^{e}$ SÉRIE - TOME $39-2006-\mathrm{N}^{\circ} 1$ 
Comme la restriction à $Z$ d'un $R G$-module projectif lisse est un $R Z$-module projectif lisse (puisque l'induction lisse est un adjoint à droite exact de la restriction), on a des isomorphismes $L^{q}(? \mapsto ? Z) \simeq H_{q}(Z, ?)$ (groupes d'homologie) pour tout $R G$-module lisse ?. On en déduit une suite spectrale $\left(E_{r}^{p q}\right)_{r \in \mathbb{N}}$ de deuxième terme

$$
E_{2}^{p q}=\operatorname{Ext}_{G / Z}\left(H_{q}\left(Z, \pi_{J}^{R}\right) \pi_{I}^{R}\right)
$$

convergeant vers le gradué de $\operatorname{Ext}_{G}^{p+q}\left(\pi_{J}^{R}, \pi_{I}^{R}\right)$ pour une certaine filtration.

Or, on a des isomorphismes de $R(G / Z)$-modules lisses

$$
H_{q}\left(Z, \pi_{J}^{R}\right) \simeq \pi_{J}^{R} \otimes H_{q}(Z, R)
$$

le terme à la droite du $\otimes$ étant muni de l'action triviale de $G / Z$. Par le théorème précédent, il s'ensuit que seule la colonne $p=\delta(I, J)$ du terme $E_{2}^{p q}$ est non nulle, et par conséquent la suite spectrale dégénère fortement en un isomorphisme

$$
E_{2}^{\delta(I, J) q}=H_{q}(Z, R) \stackrel{\sim}{\longrightarrow} \operatorname{Ext}_{G}^{\delta(I, J)+q}\left(\pi_{J}^{R}, \pi_{I}^{R}\right) .
$$

Rappelons maintenant que $Z \simeq \mathbb{Z}^{d} \times Z^{0}$ où $Z^{0}$ est le sous-groupe compact maximal de $Z$, dont le pro-ordre est supposé inversible dans $R$. Ainsi on a un isomorphisme

$$
H_{q}(Z, R) \stackrel{\sim}{\longrightarrow} H_{q}\left(\mathbb{Z}^{d}, R\right) .
$$

Maintenant un calcul classique (par le complexe de Koszul par exemple, voir [11, V.6.4(ii)]) montre que $H_{q}\left(\mathbb{Z}^{d}, R\right)$ est un $R$-module libre de dimension $\left(\begin{array}{l}d \\ q\end{array}\right)$ pour $q \leqslant d$ et est nul pour $q>d$.

\subsection{Construction explicite d'extensions}

Nous commençons cette section par un peu de combinatoire.

2.2.1. Soient $J, K$ deux sous-ensembles de $S$. Comme ci-dessus, on note

$$
\Delta(J, K):=(J \cup K) \backslash(J \cap K)
$$

la différence symétrique de $J$ et $K$ dans $S$ et $\delta(J, K)$ son cardinal. Par exemple, $\Delta(J, S)=J^{c}$, le complémentaire de $J$ dans $S$. On voit facilement que

$$
\Delta(J, \Delta(J, K))=K \quad \text { et } \quad \Delta\left(J^{c}, K\right)=\Delta(J, K)^{c},
$$

et non moins facilement que

2.2.2. Lemme. - Soient $I, J, K$ trois sous-ensembles de S. Les propriétés suivantes sont équivalentes:

(i) $\Delta(I, J) \supseteq \Delta(J, K)$,

(ii) $\Delta(I, J)=\Delta(J, K) \sqcup \Delta(K, I)$,

(iii) $\delta(I, J)=\delta(J, K)+\delta(K, I)$. 
Preuve. - Notons $\chi_{\text {? }}: S \rightarrow \mathbb{Z}$ la fonction caractéristique d'un sous-ensemble ? de $S$. On a $\chi_{\Delta(I, J)}=\chi_{I}+\chi_{J}-2 \chi_{I \cap J}$. Deux calculs immédiats montrent que chacun des deux premiers points est équivalent à l'égalité

$$
\chi_{K}-\chi_{K \cap I}-\chi_{K \cap J}+\chi_{I \cap J}=0 .
$$

Notons maintenant pour une fonction $f: S \rightarrow \mathbb{Z}$ sa «somme» $\operatorname{par} \int_{S} f:=\sum_{s \in S} f(s)$. Alors $\delta(I, J)=\int_{S} \chi_{\Delta(I, J)}$. Un nouveau calcul immédiat montre alors que le point (iii) est équivalent à l'égalité

$$
\int_{S}\left(\chi_{K}-\chi_{K \cap I}-\chi_{K \cap J}+\chi_{I \cap J}\right)=0 .
$$

Mais la fonction sommée est à valeurs $\geqslant 0$, donc la nullité de sa somme équivaut à sa nullité.

2.2.3. Soit $J \subseteq S$. On lui associe une fonction signe

$$
\begin{aligned}
\epsilon_{J}: S & \rightarrow\{ \pm 1\}, \\
\alpha & \mapsto-1 \text { si et seulement si } \alpha \in J
\end{aligned}
$$

et un cône ouvert de $X$

$$
X_{J}:=\left\{x \in X, \forall \alpha \in S, \epsilon_{J}(x)\langle x, \alpha\rangle>0\right\} .
$$

Les $X_{J}$ sont les composantes connexes de $X$ privé des hyperplans orthogonaux aux racines simples. En particulier, $X_{\emptyset}$ est la chambre de Weyl associée à $B_{+}$et $X_{S}$ est celle associée au Borel opposé à $B_{+}$. En général, une chambre de Weyl est soit contenue dans soit disjointe d'un cône $X_{J}$.

Si maintenant $J$ et $K$ sont deux sous-ensembles de $S$, et si l'on convient de surligner les adhérences pour la topologie réelle de $X$, on constate que $\overline{X_{K}} \cap \overline{X_{J}}$ est un cône fermé vectoriellement générateur (ou ce qui est équivalent, contenant un sous-ensemble ouvert) de

$$
a_{\Delta(J, K)}=\{x \in X, \forall \alpha \in \Delta(J, K),\langle x, \alpha\rangle=0\} .
$$

Plus précisément, c'est une réunion d'adhérences de chambres paraboliques de $a_{\Delta(J, K)}$ (une chambre parabolique de $a_{J}$ est par définition une composante connexe du complémentaire de l'union des hyperplans associés aux racines de $A_{J}$ dans le parabolique standard associé à $M_{J}$. C'est aussi le $a_{J}$-intérieur de l'intersection de l'adhérence d'une chambre de Weyl de $X$ avec $a_{J}$, lorsque cet intérieur est non vide. Ainsi les chambres paraboliques sont en bijection avec les sous-groupes paraboliques de $G$ de Levi $M_{J}$ ).

\subsubsection{Représentations induites d'un Borel}

Par commodité, nous allons supposer que $R$ contient une racine carrée de $p$, fixée une fois pour toutes. Ceci n'est pas nécessaire pour les constructions qui suivent mais simplifie l'exposition. Cela nous permet en particulier de définir

$$
\delta:=\delta_{B_{+}}^{-1 / 2}
$$

où $\delta_{B_{+}}: A_{\emptyset} \rightarrow R^{\times}$est le caractère-module de $B_{+}$, et de normaliser les induites paraboliques. Lorsque $B$ est un sous-groupe de Borel contenant $A_{\emptyset}$, nous notons $i_{B}^{G}(\delta)$ l'induite parabolique

$4^{e}$ SÉRIE - TOME $39-2006-\mathrm{N}^{\circ} 1$ 
normalisée de $\delta$ le long de $B$. On a $i_{B}^{G}(\delta)=\operatorname{Ind}_{B}^{G}\left(\delta \delta_{B}^{1 / 2}\right)$ ce qui montre que l'on peut définir cette induite sans racine de $p$.

Rappelons qu'on définit une « distance » $d\left(B, B^{\prime}\right)$ entre deux sous-groupes de Borel contenant $A_{\emptyset}$ par

$$
d\left(B, B^{\prime}\right):=\left|\Sigma\left(A_{\emptyset}, \operatorname{Lie}(B)\right) \backslash \Sigma\left(A_{\emptyset}, \operatorname{Lie}\left(B^{\prime}\right)\right)\right| .
$$

Enfin nous noterons $C(B) \subset X$ la chambre de Weyl de $X$ associée au sous-groupe de Borel $B$. Le lemme suivant est bien connu dans le cas $R=\mathbb{C}$.

2.2.5. Lemme. - On suppose que $R$ est un anneau fortement banal pour $G$, cf. 2.1.6 (pour $\mathbf{G}=G L(n)$, banal suffit). Soient $B, B^{\prime}$ deux sous-groupes de Borel.

(i) $\operatorname{Hom}_{G}\left(i_{B}^{G}(\delta), i_{B^{\prime}}^{G}(\delta)\right) \simeq R$ et contient un générateur canonique noté $J_{B^{\prime} \mid B}$, et appelé simplement "opérateur d'entrelacement».

(ii) Si $d\left(B, B^{\prime \prime}\right)=d\left(B, B^{\prime}\right)+d\left(B^{\prime}, B^{\prime \prime}\right)$, alors $J_{B^{\prime \prime} \mid B^{\prime}} \circ J_{B^{\prime} \mid B}=J_{B^{\prime \prime} \mid B}$.

(iii) Si $B$ et $B^{\prime}$ sont contenus dans un sous-groupe parabolique $P$ dont on note $M$ la composante de Levi (semi-standard), alors on a $J_{B^{\prime} \mid B}=i_{P}^{G}\left(J_{B_{M}^{\prime} \mid B_{M}}\right)$ où $J_{B_{M}^{\prime} \mid B_{M}}$ est l'opérateur d'entrelacement $i_{B \cap M}^{M}(\delta) \rightarrow i_{B^{\prime} \cap M}^{M}(\delta)$.

(iv) On a équivalence entre les assertions suivantes:

(a) $i_{B}^{G}(\delta)$ et $i_{B^{\prime}}^{G}(\delta)$ sont isomorphes (ou, ce qui est équivalent par (i), $J_{B^{\prime} \mid B}$ est un isomorphisme).

(b) $C(B)$ et $C\left(B^{\prime}\right)$ sont contenues dans un même $X_{J}$, pour un certain $J \subseteq S$.

(v) $\operatorname{Si} C(B) \subset X_{J}$, alors il existe une factorisation

$$
J_{\bar{B} \mid B}: i_{B}^{G}(\delta) \rightarrow \pi_{J}^{R} \hookrightarrow i_{B}^{G}(\delta) .
$$

Autrement dit, on a un isomorphisme im $J_{\bar{B} \mid B} \stackrel{\sim}{\longrightarrow} \pi_{J}^{R}$. (On note $\bar{B}$ le Borel opposé.)

Pour alléger cette section, nous reportons la preuve de ce lemme à la section 2.5. Nous fixons dorénavant l'anneau $R$ fortement banal pour $G$ et l'omettrons de la plupart des notations.

Choisissons maintenant pour chaque $J \subseteq S$ un Borel $B_{J}$ tel que $C\left(B_{J}\right) \subset X_{J}$ et posons

$$
I_{J}:=i_{B_{J}}^{G}(\delta)
$$

D'après le lemme précédent, la classe d'isomorphisme de $I_{J}$ ne dépend pas du choix de $B_{J}$ et ses endomorphismes sont tous scalaires. Toujours le lemme précédent nous fournit aussi un opérateur d'entrelacement

$$
J_{J \mid K}: I_{K} \rightarrow I_{J}
$$

dont la classe d'homothétie ne dépend pas des choix. En particulier, la classe d'isomorphisme de son image ne dépend pas non plus de ces choix.

2.2.7. Lemme. - Soient $I, J, K \subseteq S$ tels que $\Delta(I, J) \supseteq \Delta(K, J)$. Alors on a une factorisation à homothétie inversible près

$$
J_{J \mid I} \in R^{\times} \cdot\left(J_{J \mid K} \circ J_{K \mid I}\right) .
$$

Preuve. - Puisqu'on veut une factorisation à homothétie près, on peut jouer sur les choix des sous-groupes de Borel $B_{I}, B_{J}$ et $B_{K}$. Il nous suffit donc de montrer qu'on peut les choisir de sorte que

$$
J_{B_{J} \mid B_{I}}=J_{B_{J} \mid B_{K}} \circ J_{B_{K} \mid B_{I}}
$$


D'après le point (iii) du lemme 2.2.5, il nous suffit de trouver des chambres $C_{I}$ et $C_{J}$ respectivement dans $X_{I}$ et $X_{J}$ reliées par une galerie tendue dont l'une des chambres intermédiaires est dans $X_{K}$. (Nous renvoyons à [12] pour la notion de galerie tendue et l'usage simple que l'on en fait ci-dessous.)

Pour cela, on commence par choisir des chambres $C_{I}$ et $C_{K}^{1}$ telles que $\overline{C_{I}} \cap \overline{C_{K}^{1}}$ soit une chambre parabolique de $\overline{X_{I}} \cap \overline{X_{K}} \subset a_{\Delta(I, K)}$, que nous noterons $F_{I K}$. De la même manière, on choisit deux chambres $C_{J}$ et $C_{K}^{2}$ dont l'intersection des adhérences est une chambre parabolique $F_{J K}$ de $\overline{X_{J}} \cap \overline{X_{K}} \subset a_{\Delta(J, K)}$. Par définition de nos chambres paraboliques, si $x$ est un point intérieur à $F_{I K}$,

De même, si $y$ est un point intérieur à $F_{J K}$,

$$
\epsilon_{K}(\alpha)\langle x, \alpha\rangle \quad \text { est } \quad \begin{cases}>0 & \text { si } \alpha \in \Delta(I, K)^{c}, \\ =0 & \text { si } \alpha \in \Delta(I, K)\end{cases}
$$

$$
\epsilon_{K}(\alpha)\langle y, \alpha\rangle \quad \text { est } \quad \begin{cases}>0 & \text { si } \alpha \in \Delta(J, K)^{c}, \\ =0 & \text { si } \alpha \in \Delta(J, K)\end{cases}
$$

Comme $\Delta(I, K) \cap \Delta(J, K)=\emptyset$ (lemme 2.2.2), on en déduit que pour tout point $z$ du segment ouvert $] x, y[$ dans $X$, on a

$$
\epsilon_{K}(\alpha)\langle z, \alpha\rangle>0 \quad \text { pour tout } \alpha \in S,
$$

de sorte que $z \in X_{K}$. En conséquence, l'enclos des chambres $C_{I}$ et $C_{J}$, qui contient bien sûr l'enveloppe convexe de $F_{J K} \cup F_{I K}$ a une intersection non vide avec $X_{K}$, et donc avec au moins une chambre $C_{K} \subset X_{K}$. Par définition de l'enclos, il existe une galerie tendue entre $C_{I}$ et $C_{J}$ passant $\operatorname{par} C_{K}$.

\subsubsection{Intermède simplicial}

Soit $E$ un ensemble fini. On lui associe une petite catégorie $\mathcal{P}(E)$ dont les objets sont tous les sous-ensembles de $E$ et les flèches sont les inclusions. On appelle système de coefficients sur $\mathcal{P}(E)$ à valeurs dans une catégorie abélienne $\mathcal{C}$ tout foncteur contravariant $\mathcal{P}(E) \stackrel{\mathcal{V}}{\longrightarrow} \mathcal{C}$.

On veut associer un complexe de chaînes à un tel système de coefficients. Il faut pour cela choisir une orientation de $\mathcal{P}(E)$. On peut par exemple choisir un ordre total $\leqslant$ sur $E$ et définir pour $I \subset I^{\prime} \subseteq E$ tels que $I^{\prime}=I \sqcup\{e\}$ un signe $\epsilon\left(I, I^{\prime}\right):=(-1)^{|\{i \in I, i \leqslant e\}|}$.

On définit alors le complexe de chaînes $\mathcal{C}_{*}(\mathcal{P}(E), \mathcal{V})$ associé au système de coefficients $\mathcal{V}$ :

$$
0 \rightarrow \mathcal{V}(E) \stackrel{d_{|E|}}{\longrightarrow} \cdots \rightarrow \bigoplus_{|I|=n, I \subseteq E} \mathcal{V}(I) \stackrel{d_{n}}{\longrightarrow} \bigoplus_{|I|=n-1} \mathcal{V}(I) \rightarrow \cdots \rightarrow \mathcal{V}(\emptyset) \rightarrow 0
$$

où

$$
d_{n}=\bigoplus_{|I|=n} \sum_{I^{\prime} \subset I} \epsilon\left(I, I^{\prime}\right) \mathcal{V}\left(I^{\prime} \subset I\right) .
$$

Il est bien connu que le complexe de chaînes associé à un système de coefficients constant est acyclique, sauf si $E=\emptyset$.

2.2.9. Fixons dorénavant deux sous-ensembles $I, J$ de $S$. Nous allons définir un système de coefficients $\mathcal{V}_{I, J}$ sur la catégorie $\mathcal{P}(\Delta(I, J))$ à valeurs dans $\operatorname{Mod}_{R}(G)$. Pour cela, posons brutalement pour tout $K \subseteq \Delta(I, J)$

$$
\mathcal{V}_{I, J}(K):=\operatorname{im}\left(J_{J^{c} \mid \Delta(I, K)}: I_{\Delta(I, K)} \rightarrow I_{J^{c}}\right) .
$$

$4^{\mathrm{e}}$ SÉRIE - TOME $39-2006-\mathrm{N}^{\circ} 1$ 
En écrivant $K=\Delta(I, \Delta(I, K))$ et en utilisant le lemme 2.2.2, on obtient $\Delta(J, \Delta(I, K))=$ $\Delta(I, J) \backslash K$, puis en passant au complémentaire, $\Delta\left(J^{c}, \Delta(I, K)\right)=K \sqcup \Delta(I, J)^{c}$. On a donc

$$
K \subset K^{\prime} \subseteq \Delta(I, J) \Longrightarrow \Delta\left(J^{c}, \Delta(I, K)\right) \subset \Delta\left(J^{c}, \Delta\left(I, K^{\prime}\right)\right)
$$

donc d'après le lemme 2.2.7, on a une inclusion canonique

$$
\mathcal{V}_{I, J}\left(K \subset K^{\prime}\right): \mathcal{V}_{I, J}\left(K^{\prime}\right) \hookrightarrow \mathcal{V}_{I, J}(K) .
$$

On a donc ainsi défini un système de coefficients $\mathcal{V}_{I, J}$, qui dépend des choix des sous-groupes de Borel $B_{J}$ mais dont la classe d'isomorphisme n'en dépend pas. En supposant fixé un ordre total sur $S$, on obtient un complexe de chaînes $\mathcal{C}_{*}\left(\mathcal{P}(\Delta(I, J)), \mathcal{V}_{I, J}\right)$ de longueur $\delta(I, J)$. On peut augmenter ce complexe par le morphisme surjectif suivant : puisque $\operatorname{ker}\left(J_{J^{c} \mid I}\right) \subseteq \operatorname{ker}\left(J_{I^{c} \mid I}\right)$ (par le lemme 2.2.7), le morphisme $I_{I} \rightarrow \operatorname{im}\left(J_{I^{c} \mid I}\right)=\pi_{I}$ du point v) du lemme 2.2.5 se factorise en

$$
\mathcal{V}_{I, J}(\emptyset)=\operatorname{im}\left(I_{I} \stackrel{J_{J^{c} \mid I}}{\longrightarrow} I_{J^{c}}\right) \rightarrow \pi_{I} .
$$

Nous noterons $\mathcal{C}_{*}\left(\mathcal{V}_{I, J}\right)^{+}$le complexe ainsi augmenté. En faisant le changement de variable $K \mapsto \Delta(I, K)$ dans la définition du complexe de chaînes, le complexe augmenté $\mathcal{C}_{*}\left(\mathcal{V}_{I, J}\right)^{+}$ s'écrit aussi :

$$
\begin{aligned}
0 \rightarrow \pi_{J} \simeq \operatorname{im}\left(J_{J^{c} \mid J}\right) \rightarrow \cdots & \stackrel{d_{n+1}}{\longrightarrow} \bigoplus_{\begin{array}{c}
\delta(I, K)=n \\
\delta(J, K)=\delta(I, J)-n \\
\end{array}} \operatorname{im}\left(J_{J^{c} \mid K}\right) \\
& \stackrel{d_{n}}{\longrightarrow} \cdots \rightarrow \operatorname{im}\left(J_{J^{c} \mid I}\right) \rightarrow \pi_{I} \rightarrow 0,
\end{aligned}
$$

le terme $\pi_{I}$ étant en degré 1 et le terme $\pi_{J} \simeq \operatorname{im}\left(J_{J^{c} \mid J}\right)$ (cet isomorphisme vient de 2.2.5(v)) en degré $-\delta(I, J)$.

On peut maintenant énoncer une version explicite du théorème 2.1.4 :

2.2.11. THÉORÈME. - Supposons $R$ fortement banal pour $G$. Soient $I, J \subseteq S$.

(i) Le complexe augmenté $\mathcal{C}_{*}\left(\mathcal{V}_{I, J}\right)^{+}$de 2.2.10 est acyclique.

(ii) Soit $\alpha_{I, J} \in \operatorname{Ext}_{R G}^{\delta(I, J)}\left(\pi_{I}, \pi_{J}\right)$ la classe de $\mathcal{C}_{*}\left(\mathcal{V}_{I, J}\right)^{+}$. Alors pour tout $H \subseteq S$ tel que $\delta(H, J)=\delta(H, I)+\delta(I, J)$, le $\cup$-produit

$$
\alpha_{I, J} \cup-: \operatorname{Ext}_{R G}^{*}\left(\pi_{H}, \pi_{I}\right) \rightarrow \operatorname{Ext}_{R G}^{*+\delta(I, J)}\left(\pi_{H}, \pi_{J}\right)
$$

est un isomorphisme pour $* \in \mathbb{Z}$.

La preuve occupera les sections suivantes. Précisons tout de même comment cet énoncé implique le théorème 2.1.4. Cela utilise le lemme suivant :

2.2.12. LEMME. - Supposons $R$ banal pour $G$ et soit $I \subseteq S$.

(i) On a $R \stackrel{\sim}{\longrightarrow} \operatorname{End}_{R G}\left(\pi_{I}\right)$.

(ii) $\mathrm{Si} G$ est semi-simple, il existe une résolution projective de $\pi_{I}$ dans $\operatorname{Mod}_{R}(G)$ de longueur $|S|+1$.

Preuve. - Pour le point (i), choisissons un sous-groupe de Borel $B$ tel $C(B) \subset X_{I}$. D'après 2.2.5(v) on a un monomorphisme de $R$-modules

$$
\operatorname{End}_{R G}\left(\pi_{I}\right) \hookrightarrow \operatorname{Hom}_{R G}\left(i_{B}^{G}(\delta), i_{B}^{G}(\delta)\right) .
$$

Mais d'après 2.2.5(i), c'est un isomorphisme et le terme de droite est libre de rang 1 sur $R$. 
Le point (ii) est standard; rappelons les arguments. Puisque l'immeuble de Bruhat-Tits de $(\mathbf{G}, F)$ est contractile, son complexe de «chaînes à supports finis» est une résolution de longueur $|S|+1$ à gauche du $R$-module $R$. Cette résolution est $G$-équivariante lorsque $G$ est simplement connexe. Dans le cas général, il n'y a pas nécessairement d'orientation équivariante mais Schneider et Stuhler ont introduit une variante $G$-équivariante de ce complexe de chaînes, cf. le premier paragraphe de [40, II.1]. Les composantes de ce complexe sont des sommes d'induites $\operatorname{ind}_{G_{F}}^{G}(R)$ où $G_{F}$ est un sous-groupe ouvert compact de $G$. Ainsi, si on tensorise cette résolution par le $R$-module libre $\pi_{I}^{R}$ et si on la munit de l'action diagonale de $G$, on obtient une résolution de longueur $|S|+1$ de $\pi_{I}^{R}$ dont les composantes sont des sommes d'induites du type $\operatorname{ind}_{G_{F}}^{G}\left(\left(\pi_{I}^{R}\right)_{\mid G_{F}}\right)$. Ces induites sont des objets projectifs (pas de type fini) de $\operatorname{Mod}_{R}(G)$ car le pro-ordre de $G_{F}$ est inversible dans $R$.

\subsubsection{Le théorème 2.2.11 implique le théorème 2.1.4}

Commençons par remarquer le cas particulier $H=I$ de 2.2.11(ii) : on obtient un isomorphisme

$$
\alpha_{I, J} \cup-: \operatorname{Hom}_{R G}\left(\pi_{I}, \pi_{I}\right) \stackrel{\sim}{\longrightarrow} \operatorname{Ext}_{R G}^{\delta(I, J)}\left(\pi_{I}, \pi_{J}\right),
$$

ce qui, compte tenu de l'isomorphisme de 2.2.12(i), montre que $\operatorname{Ext}_{R G}^{\delta(I, J)}\left(\pi_{I}, \pi_{J}\right)$ est libre de rang 1 sur $R$ et engendré par $\alpha_{I, J}$.

Il faut maintenant montrer l'annulation des autres Ext. Toujours par le cas particulier ci-dessus du point (ii) de 2.2.11, il suffit de prouver que pour tout $I$,

$$
\operatorname{Ext}_{R G}^{*}\left(\pi_{I}, \pi_{I}\right)=0 \quad \text { dès que } *>0 .
$$

Appliquons pour cela ce point (ii) au cas $H=I=J^{c}$. On obtient les isomorphismes

$$
\operatorname{Ext}_{R G}^{*}\left(\pi_{I}, \pi_{I}\right) \stackrel{\sim}{\longrightarrow} \operatorname{Ext}_{R G}^{|S|+*}\left(\pi_{I}, \pi_{I^{c}}\right) .
$$

Or par 2.2.12(ii), le terme de droite est nul dès que $*>0$. On a donc prouvé le point (i) de 2.1.4. Le point (ii) de ce même théorème est alors une conséquence immédiate du point (ii) de 2.2.11.

\subsubsection{L'image de $J_{J \mid K}$}

Autant pour expliciter un peu les termes du complexe 2.2.10 que pour la preuve du théorème 2.2.11 dans les sections suivantes, nous voulons décrire assez précisément l'image de l'opérateur d'entrelacement $J_{J \mid K}: I_{K} \rightarrow I_{J}$ associé à deux sous-ensembles $J, K$ de $S$.

Pour cela, nous devons malheureusement compliquer encore un peu les notations. Si $T \subseteq S$, le groupe de Levi $M_{T}$ est un groupe réductif, muni d'un tore maximal, $A_{\emptyset}$, et d'une base de son système de racines, $T$, associée au sous-groupe de Borel $B_{+} \cap M_{T}$. La construction 2.1.3 permet donc d'associer à tout sous-ensemble $I$ de $T$ une représentation de $M_{T}$ que nous noterons $\pi_{I, T}$. Par exemple, $\pi_{T, T}$ est la représentation triviale de $M_{T}, \pi_{\emptyset, T}$ est sa représentation de Steinberg, et pour $T=S$ on a $M_{S}=G$ et on retrouve $\pi_{I, S}=\pi_{I}$.

2.2.15. Lemme. - Fixons deux sous-ensembles $J, K$ de $S$ et notons simplement $\Delta:=\Delta(J, K)$. Pour tout sous-groupe parabolique $P_{K, J}$ de $G$ associé à une chambre parabolique (cf. 2.2.3) de $a_{\Delta}$ contenue dans $\overline{X_{J}} \cap \overline{X_{K}}$, on a

$$
\operatorname{im}\left(J_{J \mid K}\right) \simeq i_{P_{K, J}}^{G}\left(\delta_{P_{\Delta}}^{-1 / 2} \pi_{K \cap \Delta, \Delta}\right) .
$$

(Rappelons que $P_{\Delta}$ est le sous-groupe parabolique standard de Levi $M_{\Delta}$.)

4 SÉRIE - TOME $39-2006-\mathrm{N}^{\circ} 1$ 
Preuve. - Comme la classe d'isomorphisme de l'image de $J_{J \mid K}$ ne dépend que de la classe d'homothétie de cet opérateur, nous pouvons jouer sur les choix de $B_{J}$ et $B_{K}$ qui interviennent dans la définition 2.2.6. Choisissons donc ces sous-groupes de Borel de sorte que chacun soit contenu dans $P_{K, J}$. Il suffit pour cela que les chambres de Weyl associées vérifient $C\left(B_{J}\right) \subset X_{J}$, $C\left(B_{K}\right) \subset X_{K}$ et $\overline{C\left(B_{K}\right)} \cap \overline{C\left(B_{J}\right)}=\overline{C\left(P_{K, J}\right)}$ en notant $C\left(P_{K, J}\right)$ la chambre parabolique de $a_{\Delta}$ associée à $P_{K, J}$.

On déduit alors du point (iii) du lemme 2.2.5 l'existence d'un diagramme commutatif

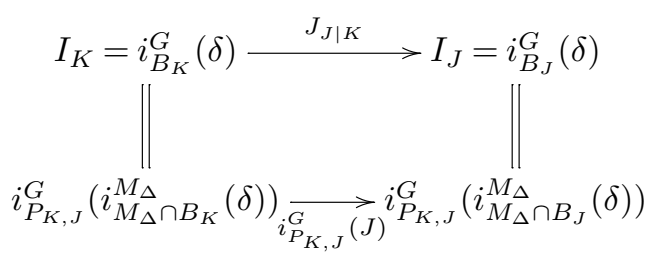

où

$$
J=J_{M_{\Delta} \cap B_{J} \mid M_{\Delta} \cap B_{K}}: i_{M_{\Delta} \cap B_{K}}^{M_{\Delta}}(\delta) \rightarrow i_{M_{\Delta} \cap B_{J}}^{M_{\Delta}}(\delta)
$$

est l'opérateur d'entrelacement canonique. On a une factorisation $\delta=\delta_{P_{\Delta}}^{-1 / 2} \delta_{M_{\Delta} \cap B_{+}}^{-1 / 2}$, et on est donc ramené à prouver que l'image de l'opérateur d'entrelacement

$$
J=J_{M_{\Delta} \cap B_{K} \mid M_{\Delta} \cap B_{J}}: i_{M_{\Delta} \cap B_{K}}^{M_{\Delta}}\left(\delta_{M_{\Delta} \cap B_{+}}^{-1 / 2}\right) \rightarrow i_{M_{\Delta} \cap B_{J}}^{M_{\Delta}}\left(\delta_{M_{\Delta} \cap B_{+}}^{-1 / 2}\right)
$$

est isomorphe à $\pi_{K \cap \Delta, \Delta}$. Mais puisque $J \cap \Delta=\Delta \backslash(K \cap \Delta)$, ceci est le point (v) du lemme 2.2.5.

2.2.16. COROLlaire. - Le système de coefficients $\mathcal{V}_{I, J}$ et le complexe augmenté $\mathcal{C}_{*}\left(\mathcal{V}_{I, J}\right)^{+}$ sont compatibles au changement de scalaires en le sens suivant : si $R \stackrel{\psi}{\longrightarrow} R^{\prime}$ est un morphisme d'anneaux fortement banals, alors les morphismes canoniques

$$
\mathcal{V}_{I, J}^{R} \otimes_{R, \psi} R^{\prime} \rightarrow \mathcal{V}_{I, J}^{R^{\prime}}, \quad \text { resp. } \quad \mathcal{C}_{*}\left(\mathcal{V}_{I, J}^{R}\right)^{+} \otimes_{R, \psi} R^{\prime} \rightarrow \mathcal{C}^{*}\left(\mathcal{V}_{I, J}^{R^{\prime}}\right)^{+}
$$

sont des isomorphismes de systèmes de coefficients sur $\Delta(I, J)$ à coefficients dans $\operatorname{Mod}_{R^{\prime}}(G)$, resp. de complexes de $R^{\prime} G$-représentations lisses.

Preuve. - Il suffit bien sûr de vérifier l'assertion pour le système de coefficients, puisque le complexe de chaînes lui est associé fonctoriellement (seule l'augmentation demande un argument supplémentaire mais qui ne pose pas de problème). Par le lemme précédent, on est ramené à prouver que pour tout $I \subseteq T \subseteq S$, l'application $\pi_{I, T}^{R} \otimes_{R, \psi} R^{\prime} \rightarrow \pi_{I, T}^{R^{\prime}}$ est un isomorphisme de $R^{\prime} G$-représentations. Mais cela résulte de l'exactitude à droite du produit tensoriel et de la définition de $\pi_{I, T}$ comme conoyau de la flèche

$$
\bigoplus_{T \supseteq J \supset I} \operatorname{Ind}_{P_{J} \cap M_{T}}^{M_{T}}(1) \rightarrow \operatorname{Ind}_{P_{I} \cap M_{T}}^{M_{T}}(1)
$$

puisque cette flèche est clairement compatible à l'extension des scalaires.

\subsection{Le cas $R$ corps algébriquement clos}

Dans cette section on suppose que $R$ est un corps algébriquement clos de caractéristique $\neq p$, dans lequel on choisit une racine de $p$ pour normaliser les foncteurs paraboliques. Pour un sous- 
groupe parabolique $P$ de Levi $M$, on notera $i_{P}^{G}$ et $r_{G}^{P}$ les foncteurs d'induction et restriction paraboliques normalisés.

La caractéristique de $R$ sera toujours supposée banale, cf. 2.1.6. La raison essentielle vient du résultat suivant :

2.3.1. FAIT. - Appelons «bloc principal» de $\operatorname{Mod}_{R}(G)$ la sous-catégorie pleine des objets dont tous les sous-quotients irréductibles sont de la série principale, c'est-à-dire apparaissent comme sous-quotients d'induites $i_{B}^{G}(\chi)$ pour $\chi$ caractère non ramifié de $T$. Lorsque $R$ est de caractéristique banale pour $G$, alors cette sous-catégorie est «facteur direct» de $\operatorname{Mod}_{R}(G)$, et les restrictions des foncteurs $r_{G}^{B}$, pour B un sous-groupe de Borel, y sont fidèles.

Preuve. - Lorsque $R=\mathbb{C}$, ceci est une partie du théorème principal de Bernstein dans [9]. La preuve est une élaboration du fait que si une représentation irréductible complexe est cuspidale, c'est-à-dire annulée par tous les foncteurs de restriction parabolique, alors elle est projective dans la catégorie des représentations complexes à caractère central fixé. Cette propriété des cuspidales n'est pas vraie en toutes caractéristiques, mais d'après [43], elle l'est en caractéristiques banales. À partir de là, il est communément admis (voir par exemple [44]) que les arguments de Bernstein s'appliquent de la même manière que dans le cas complexe.

\subsubsection{Séries principales elliptiques}

Nous allons tout d'abord préciser et renforcer le lemme 2.2.5 dans le cas d'un corps. Appelons «séries principales elliptiques » les sous-quotients irréductibles de l'induite $\operatorname{Ind}_{B_{+}}^{G}(1)=i_{B_{+}}^{G}(\delta)$ (bien que cette terminologie soit peut-être un peu usurpée lorsque $\mathbf{G} \neq G L(n)$ ). Dans le cas $R=\mathbb{C}$, elles sont classifiées par les sous-ensembles de $S$, comme on peut le déduire de plusieurs travaux plus ou moins indépendants dont ceux de Rodier dans [36], Langlands («quotient de Langlands») ou Bernstein-Zelevinski. Pour $G L(n)$, Vignéras les a classifiées en toutes caractéristiques $(\neq p)$ et dans le cas (fortement) banal, on obtient encore une paramétrisation par les sous-ensembles de $S$. Toutes ces approches montrent en particulier que ces représentations sont toutes de la forme $\pi_{I}^{R}$ définie en 2.1.3. Le lemme suivant montre entre autres qu'il en est bien de même sur $R$ de caractéristique fortement banale pour $G$.

2.3.3. Lemme. - $R$ est un corps algébriquement clos de caractéristique fortement banale (pour $\mathbf{G}=G L(n)$, «banale » suffit).

(i) Pour tout sous-groupe de Borel B, l'induite $i_{B}^{G}(\delta)$ a un unique quotient irréductible et toute série principale elliptique est isomorphe à un tel quotient.

(ii) On a équivalence entre les assertions suivantes:

(a) $i_{B}^{G}(\delta)$ et $i_{B^{\prime}}^{G}(\delta)$ sont isomorphes

(b) leurs quotients irréductibles sont isomorphes

(c) $C(B)$ et $C\left(B^{\prime}\right)$ sont contenues dans un même $X_{J}$, pour $J \subset S$.

(iii) Pour tout $J \subseteq S$, $\pi_{J}^{R}$ est irréductible. Si $C(B) \subset X_{J}$, alors le quotient irréductible de $i_{B}^{G}(\delta)$ est isomorphe à $\pi_{J}^{R}$.

(iv) Soit $w_{0}$ l'élément de plus grande longueur de $W_{G}$. Alors la contragrédiente de $\pi_{J}^{R}$ est $\pi_{-w_{0}(J)}^{R}$.

La preuve de ce lemme est donnée avec celle de 2.2.5 dans la section 2.5. Remarquons que tous les points peuvent être mis en défaut en caractéristique non banale, même l'irréductibilité des $\pi_{I}^{R}$.

2.3.4. Remarque (Paramètres de Langlands de $\pi_{J}^{\mathbb{C}}$ ). - On suppose ici que $R=\mathbb{C}$ et $\mathbf{G}=G L(n)$. D'après le lemme précédent, $\pi_{J}$ est l'unique quotient irréductible de $\operatorname{im}\left(J_{K \mid J}\right)$ pour tout $K$ et donc en particulier pour $K=S$. Or d'après le lemme 2.2.15 on a $\operatorname{im}\left(J_{S \mid J}\right) \simeq$ $i \frac{G}{P_{J^{c}}}\left(\delta_{P_{J^{c}}}^{-1 / 2} \pi_{J \cap J^{c}, J^{c}}\right)$ et on peut expliciter $\pi_{J \cap J^{c}, J^{c}}=S t_{M_{J^{c}}}$ (représentation de Steinberg de 
$M_{J^{c}}$ ). Comme $\overline{P_{J^{c}}}$ est conjugué à $P_{-w_{0}\left(J^{c}\right)}$ par l'élément de plus grande longueur $w_{0}$ de $W_{G}$, on obtient que $\pi_{J}$ est l'unique quotient irréductible de l'induite $i_{P_{-w_{0}\left(J^{c}\right)}^{G}}\left(\delta_{P_{-w_{0}\left(J^{c}\right)}}^{1 / 2} S t_{M_{-w_{0}\left(J^{c}\right)}}\right)$. Ceci donne les paramètres de Langlands de $\pi_{J}$.

\subsubsection{Exposants}

Puisque $R$ est un corps algébriquement clos de caractéristique $\neq p$, on sait que toute $R$-représentation lisse irréductible a un caractère central. En particulier, lorsque $\pi$ est une $R$-représentation de longueur finie de $M_{J}$, on note $\operatorname{Exp}\left(A_{J}, \pi\right)$ l'ensemble des caractères centraux des sous-quotients iréductibles de $\pi$. Rappelons que dans ces circonstances on a une décomposition canonique, dite «décomposition isotypique»,

$$
\pi \simeq \bigoplus_{\chi \in \operatorname{Exp}\left(A_{J}, \pi\right)} \pi_{\chi}, \quad \text { où } \pi_{\chi}=\left\{v \in \pi, \exists n \in \mathbb{N}, \forall z \in A_{J},(\pi(z)-\chi(z))^{n} v=0\right\} .
$$

On a aussi

$$
\operatorname{Exp}\left(A_{J}, \pi\right)=\left\{\chi: A_{J} \rightarrow R^{\times}, \operatorname{Hom}_{A_{J}}(\chi, \pi) \neq 0\right\} .
$$

2.3.6. Lemme. - Soient $K, J \subseteq S$. Les propriétés suivantes pour $I \subseteq S$ sont équivalentes :

(i) $\operatorname{Hom}_{G}\left(I_{I}, \operatorname{im}\left(J_{J \mid K}\right)\right) \neq 0$,

(ii) $\pi_{I}$ est un sous-quotient irréductible de $\operatorname{im}\left(J_{J \mid K}\right)$,

(iii) $\Delta(I, J) \supseteq \Delta(J, K)$.

Preuve. - L'implication (iii) $\Rightarrow$ (i) est une conséquence de la propriété de factorisation à homothétie près du lemme 2.2.7. L'implication (i) $\Rightarrow$ (ii) est immédiate puisque $\pi_{I}$ est l'unique quotient irréductible de $I_{I}$, d'après le lemme 2.3.3.

Il nous reste à prouver (ii) $\Rightarrow$ (iii). Pour cela, nous allons d'abord calculer $\operatorname{Exp}\left(T, r_{G}^{B_{J}}\left(\pi_{I}\right)\right)$. Par définition de $\pi_{I}$, cet ensemble de caractères lisses de $T$ est contenu dans l'orbite $W . \delta$ de $\delta$. En utilisant la réciprocité de Frobenius-Casselman [45, II.3.8-2] pour la première ligne ci-dessous, on a

$$
\begin{aligned}
\operatorname{Hom}_{R T}\left(w(\delta), r_{G}^{B_{J}}\left(\pi_{I}\right)\right) \neq 0 & \Longleftrightarrow \operatorname{Hom}_{R G}\left(i \frac{G}{B_{J}}(\delta), \pi_{I}\right) \neq 0 \\
& \Longleftrightarrow \operatorname{Hom}_{R G}\left(i_{w^{-1}\left(\overline{B_{J}}\right)}^{G}(\delta), \pi_{I}\right) \neq 0 \\
& \Longleftrightarrow w^{-1}\left(-C_{J}\right) \subset X_{I}
\end{aligned}
$$

grâce à 2.3.3(iv). On a donc

$$
\begin{aligned}
\operatorname{Exp}\left(T, r_{G}^{B_{J}}\left(\pi_{I}\right)\right) & =\left\{w(\delta), w^{-1}\left(-C_{J}\right) \subset X_{I}\right\} \\
& =\left\{w(\delta), \forall x \in-C_{J}, \forall \alpha \in S, \epsilon_{I}(\alpha)\langle x, w(\alpha)\rangle>0\right\} .
\end{aligned}
$$

On peut paraphraser la dernière égalité en introduisant le cône polaire $D_{J}$ de $C_{J}$ défini par $D_{J}=\left\{y \in X^{*}, \forall x \in C_{J},\langle x, y\rangle>0\right\}$. C'est aussi le cône engendré par les racines simples correspondant à la chambre de Weyl $C_{J}$ (on appelle parfois $D_{J}$ la chambre obtuse). On peut donc réécrire

$$
\operatorname{Exp}\left(T, r_{G}^{B_{J}}\left(\pi_{I}\right)\right)=\left\{w(\delta), \forall \alpha \in S, \epsilon_{I}(\alpha) w(\alpha) \in-D_{J}\right\} .
$$

Dans la suite, on utilise les notations du lemme 2.2.15. Pour alléger un peu ces notations, on pose $\sigma_{J}^{K}:=\delta_{P_{\Delta}}^{-1 / 2} \pi_{K \cap \Delta, \Delta}$. Soit $P_{K, J}$ un sous-groupe parabolique comme dans 2.2 .15 et $B_{J}, B_{K} \subset P_{K, J}$ deux sous-groupes de Borel tels que $C\left(B_{J}\right) \subset X_{J}$ et $C\left(B_{K}\right) \subset X_{K}$. On va 
maintenant calculer $\operatorname{Exp}\left(r_{G}^{B_{J}} \circ i_{P_{K, J}}^{G}\left(\sigma_{J}^{K}\right)\right)$. Pour utiliser le lemme géométrique, introduisons le sous-ensemble

$$
W^{J}=\left\{w \in W, w\left(D_{J}^{\Delta}\right) \subset D_{J}\right\}
$$

où $D_{J}^{\Delta}$ est le cône de $\left(a_{\Delta(J, K)}\right)^{\perp}$ engendré par l'ensemble $J \cap \Delta$ des racines simples correspondant au sous-groupe de Borel $B_{J} \cap M_{\Delta}$ de $M_{\Delta}$ (rappelons que $\Delta=\Delta(J, K)$ ). On sait $[8,2.11]$ que $W^{J}$ est un ensemble de représentants privilégiés des classes à droite de $W$ modulo $W(\Delta)$, et le lemme géométrique $[8,2.12]$ nous assure que

$$
\operatorname{Exp}\left(T, r_{G}^{B_{J}} \circ i_{P_{K, J}}^{G}\left(\sigma_{J}^{K}\right)\right)=\bigsqcup_{v \in W^{J}} v \cdot \operatorname{Exp}\left(T, r_{M_{\Delta}}^{M_{\Delta} \cap B_{J}} \sigma_{J}^{K}\right) .
$$

Par ailleurs, la définition de $\sigma_{J}^{K}$ nous permet de calculer comme (2.3.7)

$$
\begin{aligned}
& \operatorname{Exp}\left(T, r_{M_{\Delta}}^{M_{\Delta} \cap B_{J}} \sigma_{J}^{K}\right) \\
& \quad=\left\{w_{\Delta}(\delta), w_{\Delta} \in W(\Delta) \text { et } \forall \alpha \in \Delta, \epsilon_{K}(\alpha) w_{\Delta}(\alpha) \in-D_{J}^{\Delta}\right\} .
\end{aligned}
$$

Supposons maintenant que $\pi_{I}$ soit un sous-quotient irréductible de $\operatorname{im}\left(J_{J \mid K}\right)$. En particulier, on doit avoir

$$
\operatorname{Exp}\left(T, r_{G}^{B_{J}}\left(\pi_{I}\right)\right) \subset \operatorname{Exp}\left(T, r_{G}^{B_{J}} \circ i_{P_{K, J}}^{G}\left(\sigma_{J}^{K}\right)\right) .
$$

Fixons alors $\chi$ dans le terme de gauche. Par (2.3.8) et (2.3.9), on peut écrire $\chi=v w_{\Delta}(\delta)$ avec $v \in W^{J}$ et $w_{\Delta} \in W(\Delta)$.

Soit $\alpha \in \Delta$, par (2.3.9), on a $\epsilon_{K}(\alpha) w_{\Delta}(\alpha) \in-D_{J}^{\Delta}$. Par définition de $W^{J}$, on a donc aussi $\epsilon_{K}(\alpha) v w_{\Delta}(\alpha) \in-D_{J}$. Mais par (2.3.7), ceci entraine que $\epsilon_{K}(\alpha)=\epsilon_{I}(\alpha)$. En d'autres termes : la restriction de $\epsilon_{I}$ à $\Delta(J, K)$ est l'opposée de celle de $\epsilon_{J}$. Ou autrement dit, $\Delta(I, J) \supseteq$ $\Delta(J, K)$.

\subsubsection{Acyclicité des complexes $\mathcal{C}_{*}\left(\mathcal{V}_{I, J}\right)^{+}$de (2.2.10)}

Fixons deux sous-ensembles $I, J$ de $S$. Nous voulons ici montrer, sous l'hypothèse que $R$ est un corps algébriquement clos de caractéristique fortement banale, l'acyclicité du complexe de chaînes augmenté $\mathcal{C}_{*}\left(\mathcal{V}_{I, J}\right)^{+}$. Comme la caractéristique de $R$ est banale, le foncteur $r_{G}^{B_{+}}$ est exact et fidèle sur le bloc principal, cf. 2.3.1. Il suffit donc de vérifier que $r_{G}^{B_{+}}\left(\mathcal{C}_{*}\left(\mathcal{V}_{I, J}^{+}\right)\right)$est exact. Ce dernier complexe est un complexe de représentations non-ramifiées de longueur finie du tore maximal $T$, et se décompose donc en une somme directe de ses composantes $\chi$-isotypiques, pour $\chi$ caractère non ramifié de $T$.

$$
r_{G}^{B_{+}}\left(\mathcal{C}_{*}\left(\mathcal{V}_{I, J}\right)^{+}\right)=\bigoplus_{\chi \in W . \delta}\left(r_{G}^{B_{+}} \mathcal{C}_{*}\left(\mathcal{V}_{I, J}\right)^{+}\right)_{\chi}
$$

Pour calculer ces composantes isotypiques, on remarque d'abord que les représentations $r_{G}^{B_{+}}\left(\operatorname{im}\left(J_{J \mid K}\right)\right)$ sont toutes de multiplicité 1, et donc semi-simples. Il s'ensuit simplement que

$$
\left(r_{G}^{B_{+}} \mathcal{C}_{*}\left(\mathcal{V}_{I, J}\right)^{+}\right)_{\chi} \simeq \operatorname{Hom}_{T}\left(\chi, r_{G}^{B_{+}} \mathcal{C}_{*}\left(\mathcal{V}_{I, J}\right)^{+}\right) .
$$

Posons maintenant pour tout $K \subseteq \Delta(I, J)$

$$
\mathcal{V}_{I, J, \chi}(K):=\operatorname{Hom}_{T}\left(\chi, r_{G}^{B_{+}} \mathcal{V}_{I, J}(K)\right) .
$$

$4^{\mathrm{e}}$ SÉRIE - TOME $39-2006-\mathrm{N}^{\circ} 1$ 
Les flèches de transition $\mathcal{V}_{I, J}\left(K \subset K^{\prime}\right)$ induisent par fonctorialité des flèches

$$
\mathcal{V}_{I, J, \chi}\left(K \subset K^{\prime}\right): \mathcal{V}_{I, J, \chi}\left(K^{\prime}\right) \rightarrow \mathcal{V}_{I, J, \chi}(K)
$$

et on obtient ainsi un système de coefficients $\mathcal{V}_{I, J, \chi}$ sur la catégorie $\mathcal{P}(\Delta(I, J))$ à valeurs dans la catégorie des $R$-espaces vectoriels. On peut augmenter le complexe de chaînes $\mathcal{C}_{*}\left(\mathcal{V}_{I, J, \chi}\right)$ associé par l'application

$$
\mathcal{V}_{I, J, \chi}(\emptyset)=\operatorname{Hom}_{T}\left(\chi, r_{G}^{B_{+}}\left(I_{I}\right)\right) \rightarrow \operatorname{Hom}_{T}\left(\chi, r_{G}^{B_{+}}\left(\pi_{I}\right)\right)
$$

et en notant $\mathcal{C}_{*}\left(\mathcal{V}_{I, J, \chi}\right)^{+}$le complexe ainsi augmenté, on a bien sûr

$$
\mathcal{C}_{*}\left(\mathcal{V}_{I, J, \chi}\right)^{+}=\left(r_{G}^{B_{+}} \mathcal{C}_{*}\left(\mathcal{V}_{I, J}\right)^{+}\right)_{\chi}
$$

Il nous suffit donc de montrer que pour tout $\chi$ le complexe de gauche est acyclique.

Commençons par remarquer que les flèches de transition $\mathcal{V}_{I, J, \chi}\left(K \subset K^{\prime}\right)$ sont injectives (par exactitude de $r_{G}^{B_{+}}$et exactitude à gauche de $\operatorname{Hom}_{T}(\chi,$.$) , et parce que les flèches \mathcal{V}_{I, J}\left(K \subset K^{\prime}\right)$ le sont). Comme les espaces $\mathcal{V}_{I, J, \chi}(K)$ sont de dimension 0 ou 1 , il nous suffira donc de préciser cette dimension pour déterminer le système de coefficients $\mathcal{V}_{I, J, \chi}$. Pour cela, notons $L$ l'unique sous-ensemble de $S$ tel que $I_{L} \simeq i \frac{G}{B_{+}}(\chi)$ (ou ce qui est équivalent, tel que $\pi_{L}$ soit un quotient de $i \frac{G}{B_{+}}(\chi)$ ). Par réciprocité de Frobenius-Casselman [45, II.3.8-2], on a $\mathcal{V}_{I, J, \chi}(K) \neq 0$ si et seulement si $\operatorname{Hom}_{G}\left(I_{L}, \operatorname{im}\left(J_{J^{c} \mid \Delta(I, K)}\right)\right) \neq 0$. D'après le lemme 2.3.6, ceci est encore équivalent à $\Delta\left(L, J^{c}\right) \supseteq \Delta\left(J^{c}, \Delta(I, K)\right)=K \sqcup \Delta\left(I, J^{c}\right)$ (la dernière égalité a été expliquée lors de la définition des $\left.\mathcal{V}_{I, J}\right)$.

En particulier, le système de coefficients $\mathcal{V}_{I, J, \chi}$ et son complexe augmenté ne sont non nuls que si $\Delta\left(L, J^{c}\right) \supseteq \Delta\left(I, J^{c}\right)$. Supposons tout d'abord que $\Delta\left(L, J^{c}\right) \supsetneq \Delta\left(I, J^{c}\right)$. Dans ce caslà, $L \neq I$ donc le complexe augmenté $\mathcal{C}_{*}\left(\mathcal{V}_{I, J, \chi}\right)^{+}$coöncide avec le complexe non-augmenté car $\operatorname{Hom}_{T}\left(\chi, r_{G}^{B_{+}}\left(\pi_{I}\right)\right)=0$. Or, la discussion précédente montre que le système de coefficients $\mathcal{V}_{I, J, \chi}$ est supporté par le sous-simplexe des sous-ensembles de $\Delta\left(L, J^{c}\right) \backslash \Delta\left(I, J^{c}\right)=\Delta(I, L)$ (lemme 2.2.2) et y est constant. En d'autres termes

$$
\mathcal{C}_{*}\left(\mathcal{P}(\Delta(I, J)), \mathcal{V}_{I, J, \chi}\right) \simeq \mathcal{C}_{*}(\mathcal{P}(\Delta(I, L)), R)
$$

et comme on l'a déja rappelé, le complexe de droite est acyclique, puisque $I \neq L$.

Supposons maintenant $\Delta\left(L, J^{c}\right)=\Delta\left(I, J^{c}\right)$, c'est-à-dire $L=I$. Alors le système de coefficients $\mathcal{V}_{I, J, \chi}$ est supporté par $\{\emptyset\}$ mais l'augmentation est ici un isomorphisme, de sorte que

$$
\mathcal{C}_{*}\left(\mathcal{V}_{I, J, \chi}\right)^{+} \simeq(R \stackrel{\mathrm{id}}{\longrightarrow} R)
$$

dont l'homologie est bien sûr nulle.

C'est essentiellement dans le lemme suivant qu'intervient vraiment l'hypothèse de bonne caractéristique de 2.1.5.

2.3.11. Lemme. - Supposons que la caractéristique de $R$ soit bonne pour $G$, c'est-à-dire ne divise pas l'entier $N_{W}$ de 2.1.5. Soient $J \subseteq S$ et $w \in W$ tel que $w(\delta)_{\mid A_{J}}=\delta_{\mid A_{J}}$. Alors $w \in W(J)$ où $W(J)$ est le groupe de Weyl de $M_{J}$.

Preuve. - Remarquons tout d'abord que puisque $\delta$ est trivial sur le centre de $G$, il suffit de montrer l'assertion pour $G^{a d}:=\mathbf{G} / \mathbf{Z}(\mathbf{G})(K)$. Nous supposerons donc $\mathbf{G}$ adjoint. 
Pour un élément $x$ quelconque de $X^{*}$ nous noterons $x_{J} \in a_{J}^{*}$ son image par la projection canonique $X^{*} \rightarrow a_{J}^{*}$.

Première étape : sous l'hypothèse de caractéristique, on a pour tout $J$ et tout $w$ :

$$
w(\delta)_{\mid A_{J}}=\delta_{\mid A_{J}} \Longleftrightarrow w(\rho)_{J}=\rho_{J} .
$$

Rappelons pour cela que $\delta$ est défini par $\forall t \in T, \delta(t):=q_{F}^{-\frac{1}{2} \operatorname{val}_{F}(\rho(t))}$, ou ce qui est équivalent : pour tout cocaractère $\tau: \mathbb{G}_{m} \rightarrow \mathbf{T}$,

$$
\delta\left(\tau\left(\varpi_{F}\right)\right):=q_{F}^{-\frac{1}{2}\langle\rho, \tau\rangle}
$$

où val $F, \varpi_{F}$ et $q_{F}$ sont respectivement la valuation, une uniformisante et le cardinal du corps résiduel de $F$, et $\langle\cdot, \cdot\rangle: X^{*}(\mathbf{T}) \times X_{*}(\mathbf{T})$ est l'accouplement canonique.

Comme on s'est ramené au cas adjoint, on peut considérer la famille $\left\{\omega_{\alpha}\right\}_{\alpha \in S}$ des co-poids fondamentaux. On a

$$
\begin{array}{rll}
\left(w(\delta)_{\mid A_{J}}=\delta_{\mid A_{J}}\right) & \text { si et seulement si } & \left(\forall \alpha \in J^{c}, w(\delta)\left(\omega_{\alpha}\left(\varpi_{F}\right)\right)=\delta\left(\omega_{\alpha}\left(\varpi_{F}\right)\right)\right) \\
& \text { si et seulement si } & \left(\forall \alpha \in J^{c}, q_{F}^{\frac{1}{2}\left\langle\rho-w(\rho), \omega_{\alpha}\right\rangle}=1\right) .
\end{array}
$$

D'autre part, identifiant $X_{*}(\mathbf{T})$ avec un réseau de $X$, on a

$$
w(\rho)_{J}=\rho_{J} \quad \text { si et seulement si } \quad \forall \alpha \in J^{c},\left\langle\rho-w(\rho), \omega_{\alpha}\right\rangle=0 .
$$

Il nous suffira donc de montrer que pour tout $\alpha \in S$, on a

$$
q_{F}^{\frac{1}{2}\left\langle\rho-w(\rho), \omega_{\alpha}\right\rangle}=1 \Longrightarrow\left\langle\rho-w(\rho), \omega_{\alpha}\right\rangle=0 .
$$

Pour cela, on remarque que si $w_{0}$ est l'élément de plus grande longueur de $W$, alors $\frac{1}{2}(\rho-w(\rho))$ est le déterminant de l'action de $\mathbf{T}$ sur $\operatorname{Lie}\left(\mathbf{U}_{+} \cap \mathbf{U}_{+}^{w w_{0}}\right)$, de sorte que

$$
0 \leqslant \frac{1}{2}\left\langle\rho-w(\rho), \omega_{\alpha}\right\rangle \leqslant\left\langle\rho, \omega_{\alpha}\right\rangle=n_{\alpha} .
$$

Deuxième étape : $w(\rho)_{J}=\rho_{J} \Longrightarrow w \in W(J)$.

Notons momentanément $a^{J *}$ le noyau de $X^{*} \rightarrow a_{J}^{*}$; c'est aussi le sous-espace vectoriel de $X^{*}$ engendré par les racines $\alpha \in J$. La projection $X^{*} \rightarrow a_{J}^{*}$ admet une section canonique grâce au diagramme suivant

$$
a_{J}^{*} \stackrel{\sim}{\sim} X^{*}\left(\mathbf{M}_{J}\right) \otimes \mathbb{R} \stackrel{\text { res }}{\longrightarrow} X^{*}\left(\mathbf{M}_{\emptyset}\right) \otimes \mathbb{R}=X^{*},
$$

et on notera encore $a_{J}^{*}$ l'image de cette section. $\mathrm{Si}[-,-]$ est un produit scalaire sur $X$ invariant sous le groupe de Weyl $W$, alors $a_{J}^{*}$ est l'orthogonal de $a^{J *}$. On a alors les décompositions orthogonales $\rho=\rho^{J}+\rho_{J} \in a^{J *} \oplus a_{J}^{*}$ où $\rho^{J}$ est la demi-somme des racines positives de $M_{J}$, et $w(\rho)=w(\rho)_{J}+w(\rho)^{J}$.

Soit $v$ un élément de $W(J)$ tel que $v\left(w(\rho)^{J}\right)$ soit dans la chambre de Weyl positive de $a^{J *}$, c'est-à-dire : $\forall \alpha \in J,\left[\alpha, v\left(w(\rho)^{J}\right)\right] \geqslant 0$. Comme le produit scalaire est $W$-invariant, on a $v\left(w(\rho)^{J}\right)=v(w(\rho))^{J}$ et $v(w(\rho))_{J}=w(\rho)_{J}$ donc $v(w(\rho))_{J}=\rho_{J}$ par notre hypothèse. Comme par ailleurs $v w(\rho) \in \rho+\sum_{\alpha>0} \mathbb{R}_{-} \alpha$, on obtient en soustrayant $\rho_{J}$ et compte tenu de $a^{J} \cap \sum_{\alpha>0} \mathbb{R}_{-} \alpha=\sum_{\alpha \in J} \mathbb{R}_{-} \alpha$ :

$$
v w(\rho)^{J} \in \rho^{J}+\sum_{\alpha \in J} \mathbb{R}_{-} \alpha .
$$

$4^{\text {e }}$ SÉRIE - TOME $39-2006-\mathrm{N}^{\circ} 1$ 
En prenant le produit scalaire avec $v w(\rho)^{J}$ on obtient donc :

$$
\left\|v w(\rho)^{J}\right\|^{2} \leqslant\left[v w(\rho)^{J}, \rho^{J}\right] .
$$

Mais puisque $\left\|v w(\rho)^{J}\right\|=\left\|\rho^{J}\right\|$, il s'ensuit que $v w(\rho)^{J}=\rho^{J}$.

On a donc obtenu $v w(\rho)=\rho$, ce qui équivaut à $v w=1$.

\subsection{Preuve du théorème 2.2.11}

\subsubsection{Acyclicité des complexes $\mathcal{C}_{*}\left(\mathcal{V}_{I, J}\right)^{+}$}

Nous avons déja traité le cas où $R$ est un corps algébriquement clos dans la section précédente. Nous allons nous ramener à ce cas-là. Pour cela, rappelons le

\subsubsection{FAIT. - Les R-modules sous-jacents aux représentations $\pi_{I}^{R}$ sont libres.}

Preuve. - Dans le cas $\mathbf{G}=G L(n)$ ceci est prouvé dans [38, Cor. 4.5] (et même pour $R=\mathbb{Z}$ ). L'argument de loc. cit. repose sur la décomposition de Bruhat et fonctionne de la même manière pour n'importe quel groupe déployé.

Soit maintenant $H$ un pro- $p$-sous-groupe ouvert de $G$, distingué dans un compact spécial $H_{0}$ tel que $G=H_{0} B_{+}$, et ayant des décompositions d'Iwahori relativement à chaque sous-groupe parabolique semi-standard. Ces hypothèses permettent de calculer facilement les $H$-invariants d'une induite parabolique. On sait que la famille de ces sous-groupes engendre la topologie de $G$ et il suffit donc de prouver l'acyclicité des complexes $\left(\mathcal{C}_{*}\left(\mathcal{V}_{I, J}^{R}\right)^{+}\right)^{H}$ pour tout tel $H$. Mais grâce au fait ci-dessus et au lemme 2.2.15, on s'aperçoit que le complexe $\left(\mathcal{C}_{*}\left(\mathcal{V}_{I, J}^{R}\right)^{+}\right)^{H}$ est un complexe de $R$-modules projectifs de type fini. Comme on sait, 2.2.16, que le complexe est compatible aux changements de scalaires, il nous suffit donc de traiter le cas universel $R_{u}:=\mathbb{Z}\left[\frac{1}{\sqrt{p} N_{G}}\right]$. Dans ce dernier cas, on a pour tout nombre premier $l$ banal une suite exacte de complexes

$$
0 \rightarrow \mathcal{C}_{*}\left(\mathcal{V}_{I, J}^{R_{u}}\right)^{+} \stackrel{\times l}{\longrightarrow} \mathcal{C}_{*}\left(\mathcal{V}_{I, J}^{R_{u}}\right)^{+} \rightarrow \mathcal{C}^{*}\left(\mathcal{V}_{I, J}^{R_{u} / l}\right)^{+} \rightarrow 0 .
$$

Comme $R_{u} / l$ est isomorphe à $\mathbb{F}_{l}$ ou $\mathbb{F}_{l} \times \mathbb{F}_{l}$ et qu'on a déja traité le cas d'un corps de coefficients fortement banal, le complexe de droite est acyclique. On en déduit que la multiplication par $l$ est un isomorphisme sur les $R_{u}$-modules de cohomologie $\mathcal{H}_{*}\left(\mathcal{V}_{I, J}^{R_{u}}\right)$. Comme ces derniers sont de type fini, il résulte du lemme de Nakayama qu'ils sont nuls.

\subsubsection{Cup-produits et suites spectrales}

Afin de prouver le point (ii) du théorème 2.2.11, nous rappelons quelques sorites sur les groupes d'extensions, valables dans un contexte beaucoup plus général que le nôtre.

Soit $\mathcal{C}$ une catégorie abélienne ayant assez d'injectifs et soient $\pi, \sigma$ et $\rho$ trois objets de $\mathcal{C}$. On se donne aussi un complexe acyclique

$$
C_{n}=\pi \rightarrow C_{n-1} \rightarrow \cdots \rightarrow C_{0} \rightarrow C_{-1}=\sigma .
$$

Ce complexe définit un élément $\alpha \in \operatorname{Ext}_{\mathcal{C}}^{n}(\sigma, \pi)$ et on s'intéresse au cup-produit par $\alpha$ :

$$
\alpha \cup-: \operatorname{Ext}_{\mathcal{C}}^{*}(\rho, \sigma) \rightarrow \operatorname{Ext}_{\mathcal{C}}^{n+*}(\rho, \pi) .
$$

(Lorsqu'on pense le $\cup$-produit comme une simple composition de morphismes, l'application ci-dessus est bien le $\cup$-produit à gauche par $\alpha$.) 
Nous voulons ici souligner comment ce cup-produit se lit sur la suite spectrale

$$
E_{1}^{p q}=\operatorname{Ext}_{\mathcal{C}}^{q}\left(\rho, C_{p}\right) \Longrightarrow \operatorname{Ext}_{\mathcal{C}}^{q-p}(\rho, \sigma)
$$

obtenue en appliquant le foncteur $\operatorname{Hom}_{\mathcal{C}}(\rho,-)$ à une résolution injective du complexe $C$. et en filtrant le bicomplexe obtenu par les colonnes. Pour cela, il faut se rappeler que puisque les différentielles $d_{r}$ sont de degrés $(-1-r,-r)$, il y a sur le bord $p=n$ de la suite spectrale des inclusions $E_{\infty}^{n *} \hookrightarrow E_{1}^{n *}$. D'autre part, le terme $E_{\infty}^{n *}$ est le dernier quotient de la filtration du terme $*-n$ de l'aboutissement et on a donc une projection canonique $\operatorname{Ext}_{\mathcal{C}}^{*-n}(\rho, \sigma) \rightarrow E_{\infty}^{n *}$. La composée de ces deux applications

$$
\operatorname{Ext}_{\mathcal{C}}^{*}(\rho, \sigma) \rightarrow E_{\infty}^{n, n+*} \rightarrow E_{1}^{n, n+*}=\operatorname{Ext}_{\mathcal{C}}^{n+*}(\rho, \pi)
$$

est justement le cup-produit à gauche par $\alpha$.

\subsubsection{Preuve de 2.2.11(ii)}

Nous utilisons les remarques du paragraphe 2.4.3 précédent : le complexe acyclique $\mathcal{C}_{*}\left(\mathcal{V}_{I, J}\right)^{+}$ explicité en (2.2.10) fournit la suite spectrale

$$
E_{1}^{p q}=\operatorname{Ext}_{G}^{q}\left(\pi_{H}, \bigoplus_{\substack{\delta(I, K)=p \\ \delta(J, K)=\delta(I, J)-p}} \operatorname{im}\left(J_{J^{c} \mid K}\right)\right) \Longrightarrow \operatorname{Ext}_{G}^{q-p}\left(\pi_{H}, \pi_{I}\right) .
$$

Le cup-produit à gauche par $\alpha_{I, J}$ considéré dans 2.2.11(ii) s'identifie alors à la composée

$$
\operatorname{Ext}_{G}^{*}\left(\pi_{H}, \pi_{I}\right) \rightarrow E_{\infty}^{\delta, \delta+*} \rightarrow E_{1}^{\delta, \delta+*}=\operatorname{Ext}_{G}^{\delta+*}\left(\pi_{H}, \pi_{J}\right)
$$

où on a posé $\delta:=\delta(I, J)$ (on ne confondra pas avec le caractère $\delta$ de $B_{+}$!). Nous allons montrer que pour tout $p \neq \delta$ et tout $q$, on a $E_{1}{ }^{p q}=0$. Ceci impliquera en particulier la dégénérescence en $E_{1}$ de la suite spectrale et donc le fait que la composée ci-dessus est un isomorphisme pour tout $* \in \mathbb{Z}$, ce qui est bien ce qu'on cherche à prouver.

Soit donc $K \subseteq S$ tel que

$$
\delta(I, K) \neq \delta \quad \text { et } \quad \delta(I, J)=\delta(I, K)+\delta(K, J)
$$

ou ce qui est équivalent par 2.2.2, tel que

$$
K \neq J \quad \text { et } \quad \Delta(K, J) \subseteq \Delta(I, J) .
$$

Choisissons un sous-groupe parabolique $P_{K, J^{c}}$ comme dans le lemme 2.2.15 dont nous reprenons les notations (notamment $\Delta:=\Delta\left(K, J^{c}\right)$ ). Par réciprocité de Frobenius-Shapiro (c'est une conséquence formelle de la réciprocité de Frobenius et de l'exactitude des foncteurs paraboliques) on a

$$
\operatorname{Ext}_{G}^{*}\left(\pi_{H}, \operatorname{im}\left(J_{J^{c} \mid K}\right)\right)=\operatorname{Ext}_{M_{\Delta\left(J^{c}, K\right)}}^{*}\left(r_{G}^{P_{K, J^{c}}}\left(\pi_{H}\right), \delta_{P_{\Delta}}^{-1 / 2} \pi_{K \cap \Delta, \Delta}\right) .
$$

Or d'après le lemme 2.4.5 ci-dessous, le terme de droite est non nul seulement si $\Delta\left(H^{c}, J^{c}\right)=$ $\Delta(H, J) \subseteq \Delta\left(J^{c}, K\right)$. Il est donc non nul seulement si $\Delta\left(J^{c}, K\right) \supseteq \Delta(H, J) \cup \Delta\left(J^{c}, I\right)$. Or, l'hypothèse $\delta(H, I)+\delta(I, J)=\delta(H, J)$ équivaut par 2.2.2 à $\Delta(I, J) \subseteq \Delta(H, J)$. Puisque 
$\Delta\left(J^{c}, I\right)=\Delta(J, I)^{c}$, il s'ensuit que le terme de droite est non-nul seulement si $\Delta\left(J^{c}, K\right)=S$, c'est-à-dire si $K=J$, ce que nous avons exclu.

2.4.5. Lemme. - Fixons $K, J$ et $I$ des sous-ensembles de $S$. Soit $P_{K, J}$ le sous-groupe parabolique associé à une chambre parabolique de $a_{\Delta(J, K)}$ contenue dans $\overline{X_{J}} \cap \overline{X_{K}}$ (comme dans le lemme 2.2.15). Supposons enfin que R est un anneau fortement banal pour $G$.

$$
\text { Si } \quad \operatorname{Ext}_{M_{\Delta}}^{*}\left(r_{G}^{P_{K, J}}\left(\pi_{I}\right), \delta_{P_{\Delta}}^{-1 / 2} \pi_{K \cap \Delta, \Delta}\right) \neq 0, \quad \text { alors } \Delta\left(I^{c}, J\right) \subseteq \Delta(J, K) .
$$

Preuve. - Commençons par l'observation générale suivante : si $\mathcal{C}$ est une catégorie abélienne avec assez d'injectifs et de projectifs, et $\mathfrak{Z}$ désigne le centre de la catégorie $\mathcal{C}$, alors les groupes $\operatorname{Ext}_{\mathcal{C}}^{*}(V, W)$ sont naturellement et canoniquement des $\mathfrak{Z}$-modules. De plus si $z \in \mathfrak{Z}$ agit par un scalaire sur $V$, resp. $W$, il agit par ce même scalaire $\operatorname{sur}_{\operatorname{Ext}}^{*}(V, W)$.

Stratégie : Dans notre cas on prend $\mathcal{C}=\operatorname{Mod}_{R}\left(M_{\Delta}\right)$ et on va produire, sous l'hypothèse $\Delta\left(I^{c}, J\right) \backslash \Delta(J, K) \neq \emptyset$, un élément de $R\left[A_{\Delta}\right]$ agissant par 0 sur $r_{G}^{P_{K, J}}\left(\pi_{I}\right)$ et par l'identité sur $\delta_{P_{\Delta}}^{-1 / 2} \pi_{K \cap \Delta, \Delta}$. La nullité des Ext* entre ces deux objets en résultera immédiatement.

Première étape : Tout caractère $A_{\Delta} \rightarrow R^{\times}$induit un morphisme de $R$-algèbres $R\left[A_{\Delta}\right] \rightarrow R$. C'est le cas par exemple pour les restrictions des caractères $w(\delta)$ à $A_{\Delta}$. D'après le lemme 2.3.11, on a

$$
\text { Si } \quad \operatorname{ker}_{R\left[A_{\Delta}\right]}\left(\delta_{\mid A_{\Delta}}\right)+\operatorname{ker}_{R\left[A_{\Delta}\right]}\left(w(\delta)_{\mid A_{\Delta}}\right) \subsetneq R\left[A_{\Delta}\right], \quad \text { alors } w \in W(\Delta) .
$$

En effet, supposons que la somme des deux noyaux ci-dessus est un idéal propre de $R\left[A_{\Delta}\right]$ et choisissons un idéal maximal $\mathcal{M}$ de $R$ contenant l'image de $\operatorname{ker}\left(w(\delta)_{\mid R\left[A_{\Delta}\right]}\right)$ par $\delta_{\mid A_{\Delta}}$. Alors on a

$$
\operatorname{ker}_{R / \mathcal{M}\left[A_{\Delta}\right]}\left(\delta_{\mid A_{\Delta}}\right)+\operatorname{ker}_{R / \mathcal{M}\left[A_{\Delta}\right]}\left(w(\delta)_{\mid A_{\Delta}}\right) \subsetneq R / \mathcal{M}\left[A_{\Delta}\right]
$$

où l'on note encore $\delta$ et $w(\delta)$ pour leurs composées avec la projection $R \rightarrow R / \mathcal{M}$. Puisque les deux noyaux ci-dessus sont des idéaux maximaux, ils coïncident et on a

$$
\delta_{\mid A_{\Delta}}=w(\delta)_{\mid A_{\Delta}} \bmod \mathcal{M}
$$

On peut donc appliquer 2.3.11 au corps $R / \mathcal{M}$.

Deuxième étape : Comme dans la preuve du lemme 2.2.15, on choisit un sous-groupe de Borel $B_{J}$ contenu dans $P_{K, J}$ et tel que $C_{J}:=C\left(B_{J}\right) \subset X_{J}$. Nous allons montrer que

$$
\text { Si } \quad\left\{w \in W, w\left(C_{J}\right) \subset X_{I^{c}}\right\} \cap W(\Delta) \neq \emptyset, \quad \text { alors } \Delta\left(I^{c}, J\right) \subseteq \Delta(J, K) .
$$

En effet, soit $w$ dans l'intersection ci-dessus. Puisque $w \in W(\Delta)$, il fixe $a_{\Delta(J, K)}$ points par points. On a donc $\overline{X_{I^{c}}} \cap \overline{X_{J}} \supset w\left(\overline{\left.C_{J}\right)} \cap \overline{C_{J}} \supset \overline{C_{J}} \cap a_{\Delta(J, K)}\right.$. Autrement dit, $\overline{X_{I^{c}}} \cap \overline{X_{J}}$ contient un cône vectoriellement générateur de $a_{\Delta(J, K)}$ (par notre choix de $B_{J}$ ). Comme on sait par ailleurs, 2.2.3, que $\overline{X_{I^{c}}} \cap \overline{X_{J}}$ est un cône fermé générateur de $a_{\Delta\left(J, I^{c}\right)}$, il s'ensuit que $a_{\Delta(J, K)} \subseteq a_{\Delta\left(I^{c}, J\right)}$ et donc que $\Delta\left(I^{c}, J\right) \subseteq \Delta(J, K)$.

Troisième étape : on suppose dorénavant que $\Delta\left(I^{c}, J\right) \backslash \Delta(J, K) \neq \emptyset$, de sorte que, par l'étape précédente, $\left\{w \in W, w\left(C_{J}\right) \subset X_{I^{c}}\right\} \cap W(\Delta)=\emptyset$. Appliquons alors (2.4.6) dans le cas « universel» de l'anneau $R_{u}=\mathbb{Z}\left[\frac{1}{\sqrt{p} N_{W} N_{G}}\right]$. On obtient une décomposition

$$
1_{R_{u}\left[A_{\Delta}\right]}=z_{\delta}+z^{\delta} \in \operatorname{ker}_{R_{u}\left[A_{\Delta}\right]}\left(\delta_{\mid A_{\Delta}}\right)+\prod_{w\left(C_{J}\right) \subset X_{I^{c}}} \operatorname{ker}_{R_{u}\left[A_{\Delta}\right]}\left(w(\delta)_{\mid A_{\Delta}}\right) .
$$


Comme tout anneau fortement banal $R$ reçoit $R_{u}$, les éléments $z_{\delta}$ et $z^{\delta}$ induisent des éléments correspondants dans $R\left[A_{\Delta}\right]$. Il est clair que l'action de $z_{\delta} \operatorname{sur} \delta_{P_{\Delta}}^{-1 / 2} \pi_{K \cap \Delta, \Delta}$ est nulle. Montrons que celle de $z^{\delta} \operatorname{sur} r_{G}^{P_{K, J}}\left(\pi_{I}\right)$ est nulle aussi. Par extension des scalaires, il suffit de traiter le cas «universel» $R_{u}$. Soit $H$ un pro- $p$-sous-groupe ouvert de $M_{\Delta}$, on sait que $r_{G}^{P_{K, J}}\left(\pi_{I}\right)^{H}$ est un $R_{u}$-module de type fini, puisque c'est le conoyau de la flèche

$$
\bigoplus_{L \supset I}\left(r_{G}^{P_{K, J}} \circ i_{P_{L}}^{G}\left(\delta_{P_{L}}^{-1 / 2}\right)\right)^{H} \rightarrow\left(r_{G}^{P_{K, J}} \circ i_{P_{I}}^{G}\left(\delta_{P_{I}}^{-1 / 2}\right)\right)^{H},
$$

et qu'il est sans torsion, puisque par 2.2.5(v) on a l'inclusion

$$
r_{G}^{P_{K, J}}\left(\pi_{I}\right)^{H} \hookrightarrow r_{G}^{P_{K, J}}\left(I_{I^{c}}\right)^{H}
$$

et que le terme de droite est sans torsion par la formule de Mackey. Pour voir que l'action de $z^{\delta}$ est nulle, il suffit donc de le vérifier après extension des scalaires $R_{u} \hookrightarrow \mathbb{C}$. Par fidélité du foncteur $r_{M_{\Delta}}^{B_{J} \cap M_{\Delta}}$, il suffit encore de vérifier que $z^{\delta}$ annule $r_{G}^{P_{K, J}}\left(\pi_{I}^{\mathbb{C}}\right)$. Mais ceci résulte du calcul des exposants de $r_{G}^{B_{J}}\left(\pi_{I}\right)$ sur un corps, effectué au-dessus de (2.3.7).

\subsection{Preuve des lemmes 2.2.5 et 2.3.3}

Nous utiliserons ici les bases de la théorie des opérateurs d'entrelacements sur un anneau de coefficients général qui sont décrites dans [14]. Soit $B$ un sous-groupe de Borel et $\bar{B}$ son opposé. Suivant la terminologie de loc. cit. 2.10 , un caractère $\chi$ de $A_{\emptyset}$ est dit $(B, \bar{B})$-régulier si

$$
\operatorname{Ann}_{R\left[A_{\emptyset}\right]}(\chi)+\bigcap_{1 \neq w \in W} \operatorname{Ann}_{R\left[A_{\emptyset}\right]}\left(\chi^{w}\right)=R\left[A_{\emptyset}\right] .
$$

Ici le caractère $\chi$ est vu comme un caractère de la $R$-algèbre $R\left[A_{\emptyset}\right]$ et la notation Ann désigne l'idéal annulateur d'un $R\left[A_{\emptyset}\right]$-module. Cette notion ne dépend pas du sous-groupe de Borel $B$ et on dira donc simplement que $\chi$ est «régulier». Elle est encore équivalente à

$$
\forall w \in W, \quad \operatorname{Ann}_{R\left[A_{\emptyset}\right]}(\chi)+\operatorname{Ann}_{R\left[A_{\emptyset}\right]}\left(\chi^{w}\right)=R\left[A_{\emptyset}\right] .
$$

2.5.1. Lemme. - Lorsque $R$ est fortement banal pour $G$, le caractère $\delta$ est régulier au sens ci-dessus. Pour $G=G L(n)$, «banal » suffit.

Preuve. - Lorsque $R$ est un corps, ceci est le cas particulier $J=\emptyset$ du lemme 2.3.11. Nous donnons ici une preuve pour $R$ un anneau, qui permet d'améliorer la restriction sur la caractéristique. Fixons $w \neq 1 \in W$ et soit $\alpha \in S$ telle que $w(\alpha) \neq \alpha$. Notons $\alpha^{\vee}: F^{\times} \rightarrow A_{\emptyset}$ la coracine associée à $\alpha$. Alors $\delta\left(\alpha^{\vee}(\varpi)\right)=q$ et $\delta^{w}\left(\alpha^{\vee}(\varpi)\right)=q^{l}$ où $l$ est la somme des coefficients de $w^{-1}(\alpha)$ dans la base $S$. Soit $h$ le nombre de Coxeter du système de racines de $G$, on a donc $|1-l| \leqslant h$. Supposons que l'entier $\prod_{0<r \leqslant h}\left(1-q^{r}\right)$ soit inversible dans $R$. Alors l'entier $q-q^{l}$ est aussi inversible dans $R$ de sorte que l'égalité

$$
1=\frac{\alpha^{\vee}(\varpi)-q}{q^{l}-q}+\frac{q^{l}-\alpha^{\vee}(\varpi)}{q^{l}-q}
$$

dans $R\left[A_{\emptyset}\right]$ montre bien que $\operatorname{Ann}_{R\left[A_{\emptyset}\right]}(\delta)+\operatorname{Ann}_{R\left[A_{\emptyset}\right]}\left(\delta^{w}\right)=R\left[A_{\emptyset}\right]$. L'hypothèse sur $R$ cidessus est en général plus faible que l'hypothèse «bon et banal». Pour $G L(n)$ elle est équivalente à «banal», par la formule donnant le cardinal de $G L_{n}\left(\mathbb{F}_{q}\right)$.

$4^{\text {e }}$ SÉRIE - TOME $39-2006-\mathrm{N}^{\circ} 1$ 


\subsubsection{Preuve de 2.2.5(i), (ii) et (iii)}

Fixons deux sous-groupes de Borel $B$ et $B^{\prime}$. Par réciprocité de Frobenius, on a $\operatorname{Hom}_{G}\left(i_{B}^{G}(\delta), i_{B^{\prime}}^{G}(\delta)\right) \simeq \operatorname{Hom}_{A_{\emptyset}}\left(r_{G}^{B^{\prime}} \circ i_{B}^{G}(\delta), \delta\right)$. Mais d'après le lemme géométrique [8, 2.12] et la régularité de $\delta$ du lemme précédent, on a

$$
r_{G}^{B^{\prime}} \circ i_{B}^{G}(\delta) \simeq \bigoplus_{w \in W} \delta^{w}
$$

On en déduit la première assertion de 2.2.5(i). Pour l'existence et la définition d'un opérateur d'entrelacement «canonique» dans ces circonstances, nous renvoyons à [14, 2.11]. Pour les propriétés 2.2.5(ii) et (iii) que ces opérateurs satisfont, nous renvoyons à la proposition 7.8. de loc. cit.

\subsubsection{Preuve de 2.3.3(i)}

Dans ce paragraphe, $R$ est donc un corps algébriquement clos de caractéristique bonne et banale (ou simplement banale pour $G L(n)$ ). D'après 2.3.1, on a une partition

$$
W . \delta=\bigsqcup_{\pi \in J H\left(i_{B}^{G}(\delta)\right)} \operatorname{Exp}\left(A_{\emptyset}, r_{G}^{\bar{B}}(\pi)\right)
$$

En particulier, $J H\left(i_{B}^{G}(\delta)\right)$ est sans multiplicités. Soit $\pi$ un sous-quotient irréductible de $i_{B_{+}}^{G}(\delta)$, et soit $w$ tel que $\delta^{w} \in \operatorname{Exp}\left(A_{\emptyset}, r_{G}^{\overline{B_{+}}}(\pi)\right)$. Par réciprocité de Frobenius-Casselman, on a un morphisme non nul $i_{B_{+}}^{G}\left(\delta^{w}\right)=i_{B_{+}^{w-1}}^{G}(\delta) \rightarrow \pi$, ce qui montre que $\pi$ est un quotient d'une induite du type $i_{B}^{G}(\delta)$.

Fixons maintenant $B$; alors par les mêmes arguments, une représentation irréductible $\pi$ de $G$ est un quotient de $i_{B}^{G}(\delta)$ si et seulement si $\delta \in \operatorname{Exp}\left(A_{\emptyset}, r_{G}^{\bar{B}}(\pi)\right)$. Par la partition ci-dessus, une telle représentation est unique (à isomorphisme près). Puisque $\operatorname{Hom}_{G}\left(i_{B}^{G}(\delta), i_{B_{+}}^{G}(\delta)\right) \neq 0$, c'est bien un sous-quotient de $i_{B_{+}}^{G}(\delta)$.

Remarque.-Choisissons un ordre total $\leqslant$ sur l'ensemble $\mathcal{P}(S)$ des sous-ensembles de $S$, raffinant l'ordre induit par la relation d'inclusion. On obtient une filtration décroissante $\operatorname{Fil}_{I}:=\sum_{I \leqslant K} \operatorname{Ind}_{P_{K}}^{G}(1) \operatorname{de}_{\operatorname{Ind}_{B_{+}}^{G}}^{G}(1)$ dont le gradué est $\bigoplus_{I \subseteq S} \pi_{I}^{R}$ (on peut par exemple le vérifier en calculant les exposants de $r_{G}^{B_{+}}\left(\mathrm{Fil}_{I}\right)$ ). Il s'ensuit que dans le groupe de Grothendieck on a l'égalité

$$
\operatorname{Ind}_{B_{+}}^{G}(1)=\sum_{I \subseteq S} \pi_{I}^{R}
$$

et que les $\pi_{I}^{R}$ sont à supports disjoints. En particulier, on a long $\left(\operatorname{Ind}_{B_{+}}^{G}(1)\right) \geqslant|\mathcal{P}(S)|$.

\subsubsection{Preuve de 2.3.3(ii)}

$R$ est toujours un corps algébriquement clos (fortement) banal. L'implication (a) $\Rightarrow$ (b) est tautologique. Supposons que $i_{B}^{G}(\delta)$ et $i_{B^{\prime}}^{G}(\delta)$ ont leurs uniques quotients irréductibles isomorphes et appelons $\pi$ (la classe d'isomorphisme de) ce quotient. Montrons que l'opérateur d'entrelacement $J_{B^{\prime} \mid B}$, qui est non nul, doit être surjectif. En effet, son image est non-nulle, et a pour (unique) quotient irréductible $\pi$. Si son conoyau était non-nul, il aurait aussi $\pi$ comme (unique) quotient irréductible, contredisant la multiplicité 1 dans $J H\left(i_{B^{\prime}}^{G}(\delta)\right)$. Donc $J_{B^{\prime} \mid B}$ est surjectif. On en déduit maintenant qu'il est injectif, puisque pour tout sous-groupe ouvert compact $H$ de $G$, on a $\operatorname{dim}_{R}\left(i_{B}^{G}(\delta)^{H}\right)=\operatorname{dim}_{R}\left(i_{B^{\prime}}^{G}(\delta)^{H}\right)=|B \backslash G / H|$. On a donc prouvé (b) $\Rightarrow$ (a). 
Il nous reste maintenant à montrer que $J_{B^{\prime} \mid B}$ est un isomorphisme si et seulement si $C^{\prime}:=C\left(B^{\prime}\right)$ et $C:=C(B)$ sont dans un même $X_{J}$ pour un certain $J \subseteq S$. Étudions d'abord le cas particulier où les chambres $C$ et $C^{\prime}$ sont adjacentes, le mur étant associé à une racine $r$. Le sous-groupe de Levi $M_{r}:=\mathcal{Z}_{G}(\operatorname{ker} r)$ est un groupe réductif déployé de rang 1 (dont le groupe adjoint n'est autre que $P G L(2))$. Le caractère $\delta$ est $\left(B \cap M_{r}, B^{\prime} \cap M_{r}\right)$-régulier (cf. [14, 7.8.ii)]) et on dispose donc d'un opérateur d'entrelacement non nul $J_{B_{M}^{\prime} \mid B_{M}}: i_{B \cap M_{r}}^{G}(\delta) \rightarrow i_{B^{\prime} \cap M_{r}}^{G}(\delta)$. Après avoir étudié les séries principales (en caractéristique banale) de ces groupes déployés de rang 1, voir par exemple [42], ou [14, 8.4.i)] couplé à la formule pour la mesure de Plancherel des séries principales de $S L(2)$ et $P G L(2)$, on sait que les assertions suivantes sont équivalentes:

(i) La représentation $i_{B \cap M_{r}}^{M_{r}}(\delta)$ est réductible.

(ii) L'opérateur $J_{B_{M}^{\prime} \mid B_{M}}$ n'est pas inversible.

(iii) $\delta \circ r^{\vee}=q_{F}^{ \pm \text {val }_{F}}$, égalité de caractères lisses $\mathbb{G}_{m}(F)=F^{\times} \rightarrow R^{\times}$.

Si $l(r)$ désigne la somme des coefficients de $r$ dans la base $S$, alors la dernière assertion est encore équivalente à : $q^{l(r)}=q^{ \pm 1}$. Comme on l'a déjà vu un peu plus haut, sous notre hypothèse de caractéristique banale, ceci équivaut à $l(r)= \pm 1$ ou encore $r \in \pm S$.

En utilisant maintenant 2.2.5(ii) et (iii), on déduit de la discussion précédente que $J_{B^{\prime} \mid B}$ est inversible si et seulement si il existe une galerie tendue entre $C^{\prime}$ et $C$ dont tous les murs successifs sont distincts des murs associés aux racines simples, autrement dit, une galerie incluse dans une composante connexe de $X$ privé des orthogonaux des racines simples. Une telle composante connexe est un $X_{J}$ et on a obtenu (a) $\Rightarrow$ (c). Pour la réciproque, il faut encore vérifier que deux chambres quelconques dans $X_{J}$ sont reliées par une galerie tendue dont tous les membres sont dans $X_{J}$, mais ceci résulte de la convexité de $X_{J}$.

Remarque. - Par ce que l'on vient de prouver et l'absence de multiplicité dans $J H\left(\operatorname{Ind}_{B_{+}}^{G}(1)\right)$, on obtient $\operatorname{long}\left(\operatorname{Ind}_{B_{+}}^{G}(1)\right)=|\mathcal{P}(S)|$. On déduit donc de la remarque précédente que les représentations $\pi_{I}^{R}$ sont irréductibles.

\subsubsection{Preuve de 2.2.5(iv)}

Dans ce paragraphe, $R$ est un anneau fortement banal pour $G$ ou simplement banal pour $G L(n)$. Remarquons que l'assertion (a) de 2.2.5(iv) est équivalente à « $J_{B^{\prime} \mid B}$ est un isomorphisme». Comme la formation de $i_{B}^{G}(\delta)$ et la définition de $J_{B^{\prime} \mid B}$ sont compatibles à l'extension des scalaires par un morphisme $R \rightarrow R^{\prime}$, il suffit de considérer le cas «universel» $R=\mathbb{Z}\left[\frac{1}{\sqrt{p} N_{G} N_{W}}\right]$.

Or, pour tout sous-groupe ouvert compact $H$, le sous- $R$-module $i_{B}^{G}(\delta)^{H}$ est libre de type fini. Il s'ensuit que $J_{B^{\prime} \mid B}$ est un isomorphisme si et seulement si il l'est après réduction à tout corps résiduel de $R$ et donc si et seulement si il l'est après changement des scalaires par un morphisme $R \rightarrow C$ où $C$ est algébriquement clos (et nécessairement de caractéristique banale).

Ainsi l'équivalence que l'on doit montrer est une conséquence de celle de 2.3.3(ii) montrée ci-dessus.

\subsubsection{Preuve de 2.3.3(iii) et 2.2.5(v)}

Dans ce paragraphe, $R$ est un anneau (fortement) banal qui sera parfois supposé être un corps. Nous allons commencer par traiter le cas $J=S$ des énoncés que l'on veut prouver. Dans ce cas, on peut expliciter l'opérateur $J_{B_{+} \mid \overline{B_{+}}}$par

$$
\begin{aligned}
J_{B_{+} \mid \overline{B_{+}}}: i \frac{G}{B_{+}}(\delta)=\operatorname{Ind} \frac{G}{B_{+}}\left(\delta_{\overline{B_{+}}}\right) & \rightarrow \operatorname{Ind}_{B_{+}}^{G}(1)=i_{B_{+}}^{G}(\delta), \\
f & \rightarrow \int_{G / \overline{B_{+}}} f(g) \mathrm{d} g .
\end{aligned}
$$

$4^{\text {e }}$ SÉRIE - TOME $39-2006-\mathrm{N}^{\circ} 1$ 
Sous cette forme, on remarque la factorisation

$$
J_{B_{+} \mid \overline{B_{+}}}: i \frac{G}{B_{+}}(\delta) \rightarrow 1=\pi_{S}^{R} \hookrightarrow i_{B_{+}}^{G}(\delta)
$$

qui est ce que l'on cherchait.

Fixons maintenant $J \subseteq S$ et choisissons un sous-groupe de Borel $B_{J}$ tel que $C\left(B_{J}\right) \subset X_{J}$ et $\overline{C\left(B_{J}\right)} \cap \overline{X_{\emptyset}}=a_{J} \cap \overline{X_{\emptyset}}$ (rappelons que $X_{\emptyset}$ est aussi la chambre de Weyl associée à $B_{+}$). Alors $B_{J}$ et $B_{+}$sont contenus dans le sous-groupe parabolique standard $P_{J}$ de Levi $M_{J}$ et par 2.2.5(iii), on a un diagramme commutatif

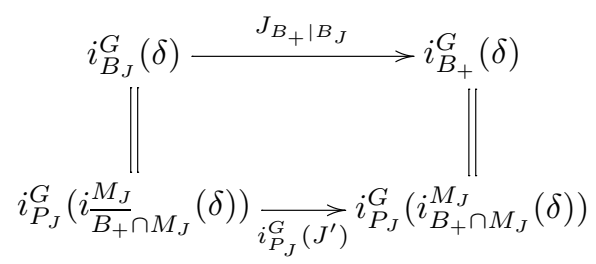

où $J^{\prime}$ est l'opérateur d'entrelacement $J_{\overline{B_{+}} \cap M_{J} \mid B_{+} \cap M_{J}}: i_{B_{+}}^{M_{J}} M_{J}(\delta) \rightarrow i_{B_{+}}^{M_{J}} M_{J}(\delta)$. Par la factorisation $\delta=\delta_{P_{J}}^{-1 / 2} \delta_{B_{+} \cap M_{J}}^{-1 / 2}$, et le cas $J=S$ traité précédemment, on obtient donc une factorisation

$$
J_{B_{+} \mid B_{J}}: i_{B_{J}}^{G}(\delta) \rightarrow i_{P_{J}}^{G}\left(\delta_{P_{J}}^{-1 / 2}\right)=\operatorname{Ind}_{P_{J}}^{G}(1) \hookrightarrow \operatorname{Ind}_{B_{+}}^{G}(1)=i_{B_{+}}^{G}(\delta) .
$$

Maintenant soit $K \supset J$. Comme dans le lemme 2.2.7 on peut trouver un sous-groupe de Borel $B_{K}$ tel que $C\left(B_{K}\right) \subset X_{K}$ et $\overline{C\left(B_{K}\right)} \cap \overline{X_{\emptyset}}=a_{K} \cap X_{\emptyset}$, et de plus $d\left(B_{K}, B_{+}\right)=$ $d\left(B_{K}, B_{J}\right)+d\left(B_{J}, B_{+}\right)$. On obtient alors le diagramme commutatif suivant :

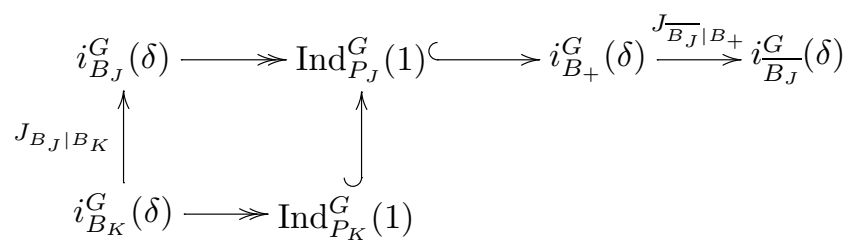

2.5.7. LEMME. - Pour $K \neq J$, on a $J_{\overline{B_{J}} \mid B_{J}} \circ J_{B_{J} \mid B_{K}}=0$.

Preuve. - Soient $B$ et $B^{\prime}$ deux sous-groupes de Borel adjacents tels que $d\left(B_{J}, B_{K}\right)=$ $d\left(B_{J}, B\right)+1+d\left(B^{\prime}, B_{K}\right)$ et tels que $C(B) \subset X_{J}$ et $C\left(B^{\prime}\right) \cap X_{J}=\emptyset$. Comme on a aussi $d\left(B_{J}, \overline{B_{J}}\right)=d\left(B_{J}, B\right)+1+d\left(B^{\prime}, \overline{B_{J}}\right)$, la composée envisagée s'écrit encore

$$
J_{\overline{B_{J}} \mid B_{J}} \circ J_{B_{J} \mid B_{K}}=J_{\overline{B_{J}} \mid B^{\prime}} \circ J_{B^{\prime} \mid B} \circ J_{B \mid B_{J}} \circ J_{B_{J} \mid B} \circ J_{B \mid B^{\prime}} \circ J_{B^{\prime} \mid B_{K}} .
$$

Par 2.2.5(i), l'endomorphisme $J_{B \mid B_{J}} \circ J_{B_{J} \mid B}$ de $i_{B}^{G}(\delta)$ est un scalaire et commute donc au reste. Ainsi la composée envisagée est de la forme

$$
J_{\overline{B_{J}} \mid B_{J}} \circ J_{B_{J} \mid B_{K}}=? \circ J_{B^{\prime} \mid B} \circ J_{B \mid B^{\prime}} \circ ?
$$

et il nous suffira de prouver que $J_{B^{\prime} \mid B} \circ J_{B \mid B^{\prime}}=0$. Par hypothèse, la racine $\alpha$ dont le mur sépare $C(B)$ et $C\left(B^{\prime}\right)$ est dans $S$. Si $P$ est le sous-groupe parabolique contenant $B$ et $B^{\prime}$, on a donc par 2.2.5(iii) 


$$
J_{B^{\prime} \mid B} \circ J_{B \mid B^{\prime}}=i_{P}^{G}\left(J_{\bar{B} \cap M_{\alpha} \mid B \cap M_{\alpha}} J_{B \cap M_{\alpha} \mid \bar{B} \cap M_{\alpha}}\right) .
$$

On est donc ramené à montrer que lorsque $G$ est de rang semi-simple 1 , on a $J_{\overline{B_{+}} \mid B_{+}}$○ $J_{B_{+} \mid \overline{B_{+}}}=0$. Lorsque $R$ est un corps algébriquement clos de caractéristique banale, ceci est bien connu. Pour passer au cas général, on se ramène au cas universel $R=\mathbb{Z}\left[\frac{1}{\sqrt{p} N_{G} N_{W}}\right]$. Dans ce dernier cas, la composée envisagée est nulle puisqu'elle l'est sur tous les points de $\operatorname{Spec} R$.

Reprenons la preuve de 2.2.5(v) et 2.3.3(iii) là où on l'a interrompue. Rappelons que $\pi_{J}$ est défini par la suite exacte

$$
\bigoplus_{K \supset J} \operatorname{Ind}_{P_{K}}^{G}(1) \rightarrow \operatorname{Ind}_{P_{J}}^{G}(1) \rightarrow \pi_{J} \rightarrow 0
$$

Ainsi par le lemme et le diagramme qui le précède, on obtient une factorisation

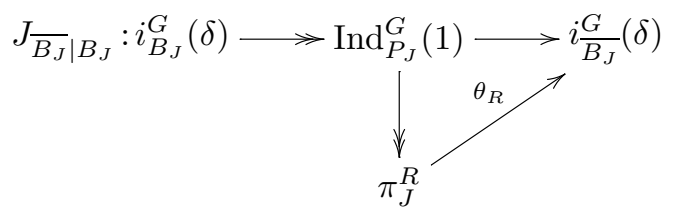

Il reste maintenant à vérifier que $\theta_{R}$ est injective. Lorsque $R$ est un corps, cela résulte de l'irréductibilité de $\pi_{J}^{R}$ (cf. les deux remarques ci-dessus) et de la non-nullité de $J_{\overline{B_{J}} \mid B_{J}}$. Pour passer au cas général, remarquons que la formation de $\pi_{J}^{R}$ et $\theta_{R}$ est compatible au changement de base. De plus on sait par 2.4.2 que pour tout sous-groupe ouvert compact, $\left(\pi_{J}^{R}\right)^{H}$ est un $R$-module libre, de même que $i_{B_{J}}^{G}(\delta)^{H}$. La propriété d'injectivité n'est pas compatible au changement de base, mais la propriété d'être un plongement localement scindable l'est. Ainsi pour montrer que $\theta_{R}$ induit un plongement localement scindable sur les $H$-invariants, on peut se ramener au cas universel $R=\mathbb{Z}\left[\frac{1}{\sqrt{p} N_{G}}\right]$, puis aux localisés de ce cas universel. Dans ce dernier cas où $R$ est local principal, la condition de scindabilité est équivalente à l'existence d'un mineur inversible pour la matrice de $\theta_{R}$ dans des bases quelconques de $\left(\pi_{J}^{R}\right)^{H}$ et $i \frac{G}{B_{J}}(\delta)^{H}$. Cette existence est assurée par réduction modulo l'idéal maximal, en utilisant le cas des corps, déja traité.

\subsubsection{Preuve de 2.3.3(iv)}

Soit $B$ tel que $C(B) \subset X_{J}$, de sorte que $\pi_{J}$ est l'unique quotient irréductible de $i_{B}^{G}(\delta)$. Alors sa contragrédiente $\pi_{J}^{\vee}$ est l'unique sous-représentation irréductible de $i_{B}^{G}\left(\delta^{-1}\right)=i_{w_{0}(B)}^{G}(\delta)$. Mais par 2.2.5(v), celle-ci est aussi l'image de $J_{w_{0}(B) \mid \overline{w_{0}(B)}}$. Donc $\pi_{J}^{\vee}$ est l'unique quotient irréductible de $i \frac{G}{w_{0}(B)}(\delta)$ dont la chambre associée est $C\left(\overline{w_{0}(B)}\right)=-w_{0}(C(B))$ et est donc contenue dans $X_{-w_{0}(J)}$. Donc $\pi_{J}^{\vee} \simeq \pi_{-w_{0}(J)}$.

\section{Cohomologie à supports compacts de $\Omega$}

Nous calculons la cohomologie à supports compacts des espaces symétriques $\Omega_{K}^{d-1}$ de Drinfeld par une méthode différente de celle de Schneider et Stuhler; au-lieu d'utiliser l'arrangement «à l'infini» des sous-variétés linéaires rationnelles de $\mathbb{P}_{K}^{d-1}$, dont le nerf est l'immeuble de Tits, nous utilisons un recouvrement de $\Omega_{K}^{d-1}$ par des ouverts «distingués » (au sens de Berkovich) dont le nerf s'identifie à l'immeuble de Bruhat-Tits.

$4^{\text {e }}$ SÉRIE - TOME $39-2006-\mathrm{N}^{\circ} 1$ 
Comme on l'a rappelé dans l'introduction, le calcul [38] concerne les groupes de cohomologie sans supports de $\Omega_{K}^{d-1}$, mais il n'est pas difficile d'en déduire «intuitivement» les groupes de cohomologie à supports compacts, sachant qu'il doivent être «lisses» et «duaux» (autant que faire se peut) des précédents. En fait, le même principe que [38] permet de calculer directement la cohomologie à supports compacts $H_{c}^{i}\left(\Omega_{K}^{d-1, \mathrm{ca}}, \Lambda\right)$ lorsque $\Lambda$ est de torsion, voir par exemple le preprint [33] d'Orlik. Mais le passage aux coefficients $l$-adiques pose de nouveaux problèmes : les $\mathbb{Z}_{l}$-modules $H_{c}^{q}\left(\Omega_{K}^{d-1, \text { ca }}, \mathbb{Z}_{l}\right)$ ne sont pas de type fini et ne sont pas la limite projective des $H_{c}^{q}\left(\Omega_{K}^{d-1, \text { ca }}, \mathbb{Z} / l^{n} \mathbb{Z}\right)$. C'est ce qui rend difficile l'adaptation de la stratégie de Schneider et Stuhler. En revanche, pour un ouvert distingué $U$ de $\mathbb{P}_{K}^{d}$, on a bien $H_{c}^{q}\left(U^{\text {ca }}, \mathbb{Z}_{l}\right)=\varliminf_{c} H_{c}^{q}\left(U^{\text {ca }}, \mathbb{Z}_{l} / l^{n} \mathbb{Z}_{l}\right)$, et c'est ce qui permettra notre calcul.

Notre approche est similaire à celle que Drinfeld a utilisée pour calculer la cohomologie de $\Omega_{K}^{1}$. Bien qu'elle semble distincte de celle de [38], les problèmes combinatoires que nous rencontrerons ont pour l'essentiel déja été résolus par Schneider et Stuhler et la lecture de cette section requiert une bonne connaissance de leurs travaux.

3.0.9. Convention. - L'anneau de coefficients $\Lambda$ sera toujours une $\mathbb{Z}_{l}$-algèbre locale de type fini, i.e. un quotient ou une localisation de l'anneau des entiers d'une extension finie de $\mathbb{Q}_{l}$, pour un entier $l \neq p$.

La définition de la cohomologie à supports compacts d'un espace analytique à valeurs dans un tel anneau est rappelée dans l'appendice B.

Nous noterons, suivant l'usage, $\mathbb{Z}_{l}(1):=\varliminf_{n}\left(\mu_{l^{n}}\left(K^{\text {ca }}\right)\right)$ le $\mathbb{Z}_{l}$-torseur des $l$-racines de l'unité. En tant que $\mathbb{Z}_{l}$-module, il est non canoniquement isomorphe à $\mathbb{Z}_{l}$, et l'action naturelle de $W_{K}$ se fait par le caractère $|-|$ qui envoie les Frobenius géométriques sur $q^{-1}$. Pour tout $\mathbb{Z}_{l}$-module on note aussi $M(1):=M \otimes_{\mathbb{Z}_{l}} \mathbb{Z}_{l}(1)$.

\subsection{Le recouvrement ouvert de Drinfeld}

Nous considérons $\Omega_{K}^{d-1}$ comme un espace de Berkovich. Rappelons sa définition, au moins en termes ensemblistes : nommons l'espace vectoriel $V:=K^{d}$ et $V^{*}$ son dual. Notons respectivement $\mathbb{A}(V)$ et $\mathbb{P}(V)$ les $K$-espaces analytiques associés par Berkovich à $V$, et $S\left(V^{*}\right)=$ $\bigoplus_{n \in \mathbb{N}} S_{n}\left(V^{*}\right)$ l'algèbre symétrique associée à $V$. Alors on a les descriptions ensemblistes

$$
\begin{gathered}
\mathbb{A}(V)=\left\{K \text {-semi-normes multiplicatives } S\left(V^{*}\right) \rightarrow \mathbb{R}_{+}\right\}, \\
\mathbb{P}(V)=(\mathbb{A}(V) \backslash\{0\}) / \sim
\end{gathered}
$$

où $x \sim y$ si et seulement si $\exists \lambda>0, \forall n \in \mathbb{N}, \forall f \in S_{n}\left(V^{*}\right),|f(x)|=\lambda^{n}|f(y)|$ (suivant Berkovich, nous notons indifféremment $x(f)=|f(x)|$ pour «la semi-norme $x$ évaluée en la fonction $f$ »). On renvoie au livre de Berkovich pour la définition des topologies et des faisceaux d'anneaux topologiques correspondants. Posons maintenant

$$
\widetilde{\Omega}(V):=\left\{x \in \mathbb{A}(V), x_{\mid V^{*}} \text { est une } K \text {-norme sur } V^{*}\right\},
$$

alors $\Omega_{K}^{d-1}$ s'identifie au sous-espace analytique $\Omega(V)$ de $\mathbb{P}(V)$ dont l'ensemble sous-jacent est l'image de $\widetilde{\Omega}(V)$.

Fixons comme dans la section précédente une paire de Borel $(B, T)$ et l'ensemble de racines simples $S$ associé. Pour décrire la cohomologie de $\Omega_{K}^{d-1}$, il convient de numéroter $S$, i.e. de fixer une bijection $S \stackrel{\sim}{\longrightarrow}\{1, \ldots, d-1\}$. On le fait de telle sorte que pour $i \in\{1, \ldots, d-1\}$, le parabolique $P_{S \backslash\{i\}}$ soit le stabilisateur d'un sous-espace de dimension $i$ de $V=K^{d}$, pour 
l'action naturelle de $G_{d}=G L_{d}(K)$ sur $K^{d}$; cela revient à numéroter la sur-diagonale «de haut en bas».

3.1.1. THÉORÈME (Schneider-Stuhler modifié). - $\Lambda$ étant comme en 3.0.9, il y a pour $i=0, \ldots, d-1$ des isomorphismes $P G L_{d}(K) \times W_{K}$-équivariants «canoniques »

$$
H_{c}^{d-1+i}\left(\Omega_{K}^{d-1, \mathrm{ca}}, \Lambda\right) \stackrel{\sim}{\longrightarrow} \pi_{\{1, \ldots, i\}}^{\Lambda}(-i) .
$$

Nous conviendrons que pour $i=0$, la représentation $\pi_{\{1, \ldots, i\}}^{\Lambda}$ est $\pi_{\emptyset}^{\Lambda}$. Nous préciserons le sens de « canonique » à la fin de la preuve.

Par la suite nous abrègerons $\Omega:=\Omega(V)=\Omega_{K}^{d-1}$ et $G:=P G L(V)$.

3.1.2. On notera $B T$ l'immeuble de Bruhat-Tits associé à $P G L\left(V^{*}\right)$ considéré comme ensemble simplicial et $|B T|$ sa réalisation géométrique, considéré comme immeuble euclidien. Rappelons la description ensembliste

$$
|B T|=\left\{\text { classes d'homothétie de } K \text {-normes sur } V^{*}\right\}
$$

qui permet de définir à moindres frais l'application de Drinfel'd

$$
\begin{aligned}
\tau: \Omega & \rightarrow|B T|, \\
x & \rightarrow x_{\mid V}: v \mapsto|v(x)| .
\end{aligned}
$$

D'après [4], cette application est surjective et continue.

3.1.3. Pour $q=0, \ldots, d$, on note $B T_{q}$ l'ensemble des $q$-simplexes de $B T$. Si $F \subset B T$ est un simplexe, on notera $|F|$ la facette de $|B T|$ associée : on a pour toute paire de simplexes $F^{\prime} \subset F \Longleftrightarrow\left|F^{\prime}\right| \subset \overline{|F|}$ où $\overline{|F|}$ désigne l'adhérence de $|F|$. Pour un simplexe $F$, on notera aussi $|F|^{*} \subset|B T|$ son lien : c'est la réunion des facettes $\left|F^{\prime}\right|$ avec $F^{\prime} \supset F$. Si $F=\left\{s_{1}, \ldots, s_{n}\right\}$ avec $s_{i} \in B T$ des sommets, on a bien sûr $|F|^{*}=\bigcap_{i}\left|s_{i}\right|^{*}$. Il s'ensuit que la famille $\left(\tau^{-1}\left(|s|^{*}\right)\right)_{s \in B T}$ de sous-ensembles de $\Omega$ est un recouvrement ouvert analytique de $\Omega$ dont le nerf est justement $B T$. De plus, chaque $\tau^{-1}\left(|s|^{*}\right)$ est distingué au sens de Berkovich, i.e. peut s'écrire comme différence de deux domaines analytiques compacts.

Le groupe $G=P G L(V)$ agit par automorphismes analytiques sur $\Omega$ et simpliciaux sur $|B T|$. L'application $\tau$ est $P G L(V)$-équivariante. Notre but est évidemment de calculer la cohomologie au moyen du recouvrement par les $\tau^{-1}\left(|s|^{*}\right)$. Comme on veut un calcul équivariant et comme il n'existe pas d'orientation $P G L(V)$-invariante sur $B T$, on modifie le complexe de Cech usuel selon la procédure introduite par Schneider-Stuhler dans $[38,40]$. En fait, pour nous raccrocher à certains résultats combinatoires de [38], nous utiliserons leur notion de systèmes de coefficients que nous rappelons ci-dessous.

\subsection{Systèmes de coefficients sur l'immeuble}

\subsubsection{Systèmes de coefficients (voir Schneider-Stuhler [38-40])}

Au complexe simplicial $B T$ est associée une catégorie $\mathcal{B T}$ dont les objets sont les simplexes et les morphismes sont induits par les inclusions de simplexes. Par définition, si $\mathcal{C}$ est une catégorie, la catégorie $\operatorname{Coef}_{B T}(\mathcal{C})$ des systèmes de coefficients à valeurs dans $\mathcal{C}$ est la catégorie des foncteurs contravariants $\mathcal{B} \mathcal{T} \rightarrow \mathcal{C}$. Celle-ci est abélienne lorsque $\mathcal{C}$ l'est. La catégorie $\mathcal{B T}$ est munie d'une action stricte de $G=P G L(V)$. Lorsque $\mathcal{C}$ est munie elle aussi d'une action (pas nécessairement stricte) de $G$, on en déduit une autre sur $\operatorname{Coef}_{B T}(\mathcal{C})$ par la formule 
$X \mapsto g_{\mathcal{B} \mathcal{T}} \circ X \circ g^{-1}{ }_{\mathcal{C}}$. La catégorie $\operatorname{Coef}_{B T}^{G}(\mathcal{C})$ des systèmes de coefficients $G$-équivariants à valeurs dans $\mathcal{C}$ est par définition la catégorie des objets $G$-équivariants de $\operatorname{Coef}_{B T} \mathcal{C}$. On rappelle qu'une structure $G$-équivariante sur un objet $X$ d'une $G$-catégorie $\mathcal{A}$ est une famille d'isomorphismes $\tau(g): g_{\mathcal{A}}(M) \stackrel{\sim}{\longrightarrow} M$ satisfaisant la condition de cocycle usuelle.

\subsubsection{Complexes de chaînes}

Schneider et Stuhler ont défini dans [40, II.1] une notion de facette orientée de l'immeuble (poly)-simplicial associé à un groupe $p$-adique quelconque. Dans le cas $P G L(V)$ qui nous intéresse, la définition est plus élémentaire, cf. [39, par. 3] : étant donnés deux ordres totaux sur un simplexe de $B T$, on les déclare équivalents s'ils se déduisent l'un de l'autre par une permutation paire des sommets. Alors un simplexe orienté est par définition un couple $(F, c)$ formé d'un simplexe et d'une classe d'équivalence d'ordres totaux sur ce simplexe. On note $B T_{(q)}$ l'ensemble des facettes orientées de dimension $q$. Si $F^{\prime} \subset F$, on note $\partial_{F^{\prime}}^{F}(c)$ l'orientation de $F^{\prime}$ induite par l'orientation $c$ de $F$.

Supposons que $\mathcal{C}$ est abélienne avec limites directes exactes. Si $X$ est un système de coefficients à valeurs dans $\mathcal{C}$, on lui associe le complexe borné d'objets de $\mathcal{C}$, dit complexe de chaînes de $X$

$$
C_{c}^{o r}(B T, X):=C_{c}^{o r}\left(B T_{(d)}, X\right) \stackrel{d_{d-1}}{\longrightarrow} \cdots \stackrel{d_{0}}{\longrightarrow} C_{c}^{o r}\left(B T_{(0)}, X\right)
$$

où

$$
C_{c}^{o r}\left(B T_{(q)}, X\right):=\operatorname{ker}(\mathrm{Id}+\alpha)
$$

avec la définition suivante de $\alpha$ : posons pour toute facette orientée $X_{(F, c)}:=X_{F}$, alors

$$
\alpha: \bigoplus_{(F, c) \in B T_{(q)}} X_{(F, c)} \rightarrow \bigoplus_{(F, c) \in B T_{(q)}} X_{(F, c)}
$$

est le morphisme induit par $X_{(F, c)} \stackrel{\mathrm{Id}}{\longrightarrow} X_{(F,-c)}$. La différentielle est induite par les morphismes

$$
\bigoplus_{F^{\prime} \subset F} X_{F^{\prime} \subset F}: X_{(F, c)} \rightarrow \bigoplus_{F^{\prime} \subset F} X_{\left(F^{\prime}, \partial_{F^{\prime}}^{F}(c)\right)}
$$

Nous noterons $\mathcal{H}_{*}(B T, X)$ les objets d'homologie du complexe $C_{c}^{o r}(B T, X)$.

Rappelons que $G$ agit sur les facettes orientées de $B T_{(q)}, q \geqslant 0$, de sorte que si $X$ est un système de coefficients $G$-équivariant, alors pour tout $q \geqslant 0$, l'objet $M:=C_{c}^{o r}\left(B T_{(q)}, X\right)$ est par construction un objet $G$-équivariant de $\mathcal{C}$. De plus les différentielles sont compatibles à ces structures $G$-équivariantes, de sorte que les objets d'homologie $\mathcal{H}_{*}(B T, X)$ sont eux-aussi $G$-équivariants.

3.2.3. Pour tout simplexe $F$ dans $B T$, on note

$$
U_{F}^{\mathrm{ca}}:=\tau^{-1}\left(|F|^{*}\right) \widehat{\otimes} \widehat{K^{\mathrm{ca}}}
$$

Si $p \in \mathbb{N}$, on vérifie que les applications

- $\left(F \in B T_{\bullet}\right) \mapsto H_{c}^{p}\left(U_{F}^{\mathrm{ca}}, \Lambda\right)$, et

- $\left(F^{\prime} \subset F\right) \mapsto\left(H_{c}^{p}\left(U_{F}^{c a}, \Lambda\right) \rightarrow H_{c}^{p}\left(U_{F^{\prime}}^{\text {ca }}, \Lambda\right)\right)$ (induit par l'immersion ouverte $\left.U_{F}^{\text {ca }} \subset U_{F^{\prime}}^{\text {ca }}\right)$, définissent un système de coefficients $G$-équivariant à valeurs dans les $\Lambda W_{K}$-modules que nous noterons simplement $F \mapsto H_{c}^{p}\left(U_{F}^{\mathrm{ca}}, \Lambda\right)$. 
3.2.4. Proposition. - Il existe une suite spectrale $G \times W_{K}$-équivariante

$$
E_{1}^{p q}=C_{c}^{o r}\left(B T_{(q)}, F \mapsto H_{c}^{p}\left(U_{F}^{\mathrm{ca}}, \Lambda\right)\right) \Longrightarrow H_{c}^{p+q}\left(\Omega^{\mathrm{ca}}, \Lambda\right)
$$

dont la différentielle $d_{1}^{p q}$ est celle du complexe de chaînes du système de coefficients $F \mapsto H_{c}^{p}\left(U_{F}^{\mathrm{ca}}, \Lambda\right)$.

Preuve. - Dans le cas de torsion, la seule complication par rapport aux arguments standard vient de ce que le nerf du recouvrement $B T$ n'a pas d'orientation $G$-invariante. C'est justement pour pallier cet inconvénient que Schneider et Stuhler ont introduit leurs facettes orientées.

Dans le cas $l$-adique, cela fonctionne de la même manière, que l'on suive la définition de Berkovich B.2.1, ou celle de Jannsen-Huber-Fargues B.2.2. Voir [19, 4.2.2] pour des arguments complets dans ce dernier cas (modulo le problème d'orientation).

3.2.5. Rappelons maintenant l'exemple essentiel de système de coefficients de $\Lambda$-modules introduit par Schneider et Stuhler. Pour $F$ simplexe, on note $\widehat{G}_{F}$ son stabilisateur, $G_{F}$ son fixateur et $G_{F}^{+}$le pro- $p$-radical de $G_{F}$ (voir par exemple [38, part 6] où le groupe $G_{F}^{+}$est noté $U_{\sigma}$ ). Partant alors d'un $\Lambda G$-module lisse $V$ on définit un système de coefficients (noté $\gamma_{0}(V)$ dans [40]) à valeurs dans la catégorie des $\Lambda$-modules :

- $F \mapsto V^{G_{F}^{+}}$.

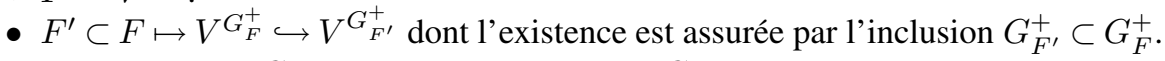

Bien sûr, l'action de $G$ sur $V$ induit une structure $G$-équivariante sur ce système de coefficients.

Le point clef de la preuve du théorème 3.1.1 est la proposition suivante qui sera prouvée dans la prochaine section :

3.2.6. Proposition. - Pour $0 \leqslant i \leqslant d-1$, il existe un isomorphisme de systèmes de coefficients $G$-équivariants en $\Lambda W_{K}$-modules

$$
\left(F \mapsto H_{c}^{d-1+i}\left(U_{F}^{c a}, \Lambda\right)\right) \stackrel{\sim}{\longrightarrow}\left(F \mapsto\left(\pi_{\{1, \ldots, i\}}^{\Lambda}\right)^{G_{F}^{+}}(-i)\right) .
$$

De plus, pour $p \notin\{d-1, \ldots, 2 d-2\}$, on a $H_{c}^{p}\left(U_{F}^{\text {ca }}, \Lambda\right)=0$ pour toute $F$.

À partir de là, le théorème 3.1.1 résulte facilement du théorème 6.8 de [38] selon lequel les complexes $C_{c}^{o r}\left(B T, F \mapsto\left(\pi_{I}^{\Lambda}\right)^{G_{F}^{+}}\right)$sont des résolutions des représentations $\pi_{I}^{\Lambda}$. En effet, la suite spectrale 3.2.4 dégénère alors en des isomorphismes

$$
E_{2}^{* 0} \stackrel{\sim}{\longrightarrow} H_{c}^{*}\left(\Omega^{\mathrm{ca}}, \Lambda\right)
$$

avec $E_{2}^{* 0}=\pi_{\{1, \ldots, i\}}^{\Lambda}(-i)$ si $*=d-1+i$ pour $0 \leqslant i \leqslant d-1$ et $E_{2}^{* 0}=0$ sinon.

\subsection{Arrangements}

Nous commençons par quelques préliminaires simpliciaux et homologiques.

\subsubsection{Ensembles partiellement ordonnés}

Soit $(\mathfrak{X}, \leqslant)$ un ensemble muni d'un ordre partiel. On peut lui associer une catégorie notée encore $\mathfrak{X}$ dont les objets sont les éléments de $\mathfrak{X}$ et les ensembles de morphismes sont singletons ou vides selon que $X \leqslant X^{\prime}$ ou non, avec une loi de composition évidente. On appelle système de coefficients sur $X$ à valeurs dans une catégorie abélienne $\mathcal{C}$ tout foncteur $\mathfrak{X} \rightarrow \mathcal{C}$.

Supposons que $\mathfrak{X}$ admet un plus petit élément $Z$ et notons $\mathfrak{X}^{(0)}:=\mathfrak{X} \backslash\{Z\}$. On peut associer à $\mathfrak{X}$ l'ensemble simplicial dont les sommets sont les éléments de $\mathfrak{X}^{(0)}$ et les $n$-simplexes sont les

$4^{\text {e }}$ SÉRIE - TOME $39-2006-\mathrm{N}^{\circ} 1$ 
sous-ensembles totalement ordonnés à $n+1$ éléments de $\mathfrak{X}^{(0)}$. Pour un tel simplexe $S$ on pose $X_{S}:=\max (S)$ l'élément maximal de $S$. On notera aussi $\mathfrak{X}^{(n)}$ l'ensemble de ces $n$-simplexes. Un système de coefficients $\mathcal{V}: X \mapsto \mathcal{V}_{X}$ sur $\mathfrak{X}$ induit un système de coefficients sur l'ensemble simplicial associé à $\mathfrak{X}$ en posant :

- $\mathcal{V}_{S}:=\mathcal{V}_{X_{S}}$, et

- pour tout $S \subset S^{\prime}$, la flèche $\iota_{S}^{S^{\prime}}: \mathcal{V}_{S} \rightarrow \mathcal{V}_{S^{\prime}}$ est donnée par la flèche associée par $\mathcal{V}$ à $X_{S} \leqslant X_{S^{\prime}}$.

Soient $S \subset S^{\prime}$ deux simplexes tels que $\left|S^{\prime}\right|=|S|+1$. On définit un signe $\epsilon_{S, S^{\prime}}:=$ $(-1)^{\operatorname{rang}_{S}\left(X^{\prime}\right)}$ où $S^{\prime}=S \cup\left\{X^{\prime}\right\}$ et $\operatorname{rang}_{S}\left(X^{\prime}\right)=\left|\left\{X \in S, X \leqslant X^{\prime}\right\}\right|$. À un système de coefficients $\mathcal{V}$ sur $\mathfrak{X}$ on associe alors le complexe de cochaînes suivant :

$$
\left(\mathcal{C}^{*}(\mathfrak{X}, \mathcal{V}), \partial^{*}\right): \mathcal{V}_{Z} \stackrel{\partial^{0}}{\longrightarrow} \bigoplus_{S \in \mathfrak{X}^{(0)}} \mathcal{V}_{S} \stackrel{\partial^{1}}{\longrightarrow} \cdots \stackrel{\partial^{k}}{\longrightarrow} \bigoplus_{S \in \mathfrak{X}^{(k)}} \mathcal{V}_{S} \stackrel{\partial^{k+1}}{\longrightarrow} \cdots
$$

où la différentielle est définie par $\partial^{0}=\sum_{X \in \mathfrak{X}(0)} \iota_{Z}^{X}$ et

$$
\partial^{k+1}=\bigoplus_{S \in \mathfrak{X}^{(k)}}\left(\sum_{S^{\prime} \in \mathfrak{X}^{(k+1)}, S^{\prime} \supset S} \epsilon_{S, S^{\prime}} \iota_{S}^{S^{\prime}}\right) \quad \text { pour } k \geqslant 0 .
$$

\subsubsection{La catégorie des arrangements}

Fixons un $K$-espace analytique $Z$ compact. On appellera arrangement dans $Z$ tout ensemble fini $\mathfrak{X}$ de sous-espaces analytiques compacts de $Z$, stable par intersection-non-vide et contenant $Z$. En particulier $\mathfrak{X}$ est un ensemble partiellement ordonné pour la contenance (c'est-à-dire $X \leqslant X^{\prime}$ si et seulement si $\left.X \supseteq X^{\prime}\right)$ muni d'un plus petit élément, $Z$. On note $U(\mathfrak{X})$ l'ouvert complémentaire $U(\mathfrak{X}):=Z \backslash \bigcup_{X \in \mathfrak{X}^{(0)}} X$, où $\mathfrak{X}^{(0)}$ a été défini au paragraphe précédent.

Supposons maintenant donné un second arrangement $\mathfrak{X}^{\prime}$. Un morphisme d'arrangements $\mathfrak{X} \rightarrow \mathfrak{X}^{\prime}$ est un couple $(f, \phi)$ où

- $f \in \operatorname{Aut}(Z)$ est un automorphisme analytique de $Z$, et

- $\phi$ une application strictement croissante $\mathfrak{X} \stackrel{\phi}{\longrightarrow} \mathfrak{X}^{\prime}$ telle que pour tout $X \in \mathfrak{X}$, on ait $\phi(X) \supseteq f(X)$.

Nous dirons aussi parfois que « $\phi$ est un morphisme d'arrangements au-dessus de $f$ ». Notons que sur les ouverts complémentaires, on a $U\left(\mathfrak{X}^{\prime}\right) \subseteq f(U(\mathfrak{X}))$.

Les arrangements de $Z$ munis de la notion de morphisme ci-dessus forment une catégorie notée $\operatorname{Arr}(Z)$ sur laquelle le groupe $\operatorname{Aut}(Z)$ des automorphismes analytiques de $Z$ agit strictement.

\subsubsection{Cohomologie des ouverts complémentaires}

On supposera par précaution que tous les espaces analytiques compacts considérés ci-dessous sont «quasi-algébriques », i.e. satisfont les hypothèses de [3, Cor. 5.6]. On sait alors que dans le cas où $\Lambda$ est $l$-adique, on a simplement $H_{c}^{p}\left(X^{\mathrm{ca}}, \Lambda\right)=\varliminf_{H^{p}}\left(X^{\mathrm{ca}}, \Lambda / \mathfrak{m}^{n}\right)$ et on en déduit les flèches contravariantes $H_{c}^{p}\left(X_{1}^{\mathrm{ca}}, \Lambda\right) \rightarrow H_{c}^{p}\left(X_{2}^{\mathrm{ca}}, \Lambda\right)$ pour $X_{2} \subset X_{1}$ par passage à la limite projective. Pour cette raison, nous noterons simplement $H^{p}$ plutôt que $H_{c}^{p}$, même dans le cas $l$-adique.

Étant donné un arrangement $\mathfrak{X}$ dans $Z$, on dispose de systèmes de coefficients $X \mapsto$ $H^{p}\left(X^{\mathrm{ca}}, \Lambda\right)$ pour $p \in \mathbb{N}$. Si on se donne un morphisme d'arrangements $(f, \phi): \mathfrak{X} \rightarrow \mathfrak{X}^{\prime}$, on a un morphisme de complexes de cochaînes

$$
\mathcal{C}^{*}(f, \phi): \mathcal{C}^{*}\left(\mathfrak{X}^{\prime}, X^{\prime} \mapsto H^{p}\left(X^{\prime \mathrm{ca}}, \Lambda\right)\right) \rightarrow \mathcal{C}^{*}\left(\mathfrak{X}, X \mapsto H^{p}\left(X^{\mathrm{ca}}, \Lambda\right)\right)
$$


donné par

$$
\mathcal{C}^{k}(f, \phi)=\bigoplus_{S^{\prime} \in \mathfrak{X}^{\prime(k)}} \sum_{S \in \phi^{-1}\left(S^{\prime}\right)} f_{S^{\prime} S}^{*}
$$

avec $f_{S^{\prime} S}^{*}: H^{p}\left(X_{S^{\prime}}^{\text {ca }}, \Lambda\right) \rightarrow H^{p}\left(X_{S}^{\text {ca }}, \Lambda\right)$ le morphisme induit par la restriction $f_{\mid X_{S}}: X_{S} \rightarrow X_{S^{\prime}}$.

3.3.4. PROPOSITION. -

(i) Il existe une suite spectrale $W_{K}$-équivariante

$$
E_{1}^{p q}(\mathfrak{X})=\mathcal{C}^{q}\left(\mathfrak{X}, X \mapsto H^{p}\left(X^{\mathrm{ca}}, \Lambda\right)\right) \Longrightarrow H_{c}^{p+q}\left(U(\mathfrak{X})^{\mathrm{ca}}, \Lambda\right)
$$

dont la différentielle $d_{1}^{p q}$ est celle du complexe de cochaînes du système de coefficients $X \mapsto H^{p}\left(X^{\mathrm{ca}}, \Lambda\right)$.

(2) Tout morphisme d'arrangements $\mathfrak{X} \stackrel{(f, \phi)}{\longrightarrow} \mathfrak{X}^{\prime}$ induit un morphisme de suites spectrales $\left(E_{r}^{p q}\right)\left(\mathfrak{X}^{\prime}\right)_{p, q, r} \rightarrow\left(E_{r}^{p q}\right)(\mathfrak{X})_{p, q, r}$ donné par le morphisme $\mathcal{C}^{*}(f, \phi)$ ci-dessus sur les termes $E_{1}^{p *}(\mathfrak{X})$ et compatible sur l'aboutissement au morphisme $H_{c}^{p+q}\left(U\left(\mathfrak{X}^{\prime}\right)^{\mathrm{ca}}, \Lambda\right) \stackrel{f_{!}^{-1}}{\longrightarrow}$ $H_{c}^{p+q}\left(U(\mathfrak{X})^{\text {ca }}, \Lambda\right)$ induit par l'immersion $f_{\mid U\left(\mathfrak{X}^{\prime}\right)}^{-1}: U\left(\mathfrak{X}^{\prime}\right) \rightarrow U(\mathfrak{X})$.

Preuve. - Tout cela est encore une fois standard dans le cas de torsion et nous omettons la preuve. Dans le cas où $\Lambda$ est fini sur $\mathbb{Z}_{l}$ d'idéal maximal $\mathfrak{m}$, il faut rappeler que puisque $U(\mathfrak{X})$ est «distingué », on a d'après [19, 4.1.9 a) et 4.1.15] des isomorphismes

$$
H_{c}^{p}\left(U(\mathfrak{X})^{\mathrm{ca}}, \Lambda\right) \stackrel{\sim}{\longrightarrow} \varliminf_{n} H_{c}^{p}\left(U(\mathfrak{X})^{\mathrm{ca}}, \Lambda / \mathfrak{m}^{n}\right) .
$$

De plus, les systèmes projectifs $\left(H^{p}\left(X^{\text {ca }}, \Lambda / \mathfrak{m}^{n}\right)\right)_{n}$ sont des systèmes de $\Lambda$-modules finis et sont donc lim-acycliques. Ainsi le cas $l$-adique se déduit du cas de torsion par simple passage à la limite.

\subsubsection{Fonctions associées à un sommet}

Après ces préliminaires simpliciaux, revenons au demi-plan. Notre but est d'exhiber pour chaque facette $F$ de l'immeuble un arrangement convenable $\mathfrak{X}_{F}$ dans $\mathbb{P}(V)$ tel que $U_{F}=$ $\mathbb{P}(V) \backslash \bigcup_{X \in \mathfrak{X}_{F}^{(0)}} X$.

Soit $s \in B T$ un sommet de l'immeuble. Choisissons un $\mathcal{O}_{K}$-réseau générateur $\mathcal{V}^{*} \subset V^{*}$ tel que la norme

$$
\begin{aligned}
|\cdot| \mathcal{V}^{*}: V^{*} & \rightarrow \mathbb{R}_{+}, \\
f & \mapsto \min \left\{|x|_{K}, x \in K^{*} \text { t.q. } x^{-1} f \in \mathcal{V}^{*}\right\}
\end{aligned}
$$

représente le sommet $s$. Posons alors $x_{\mathcal{V}^{*}}:=\sup _{f \in \mathcal{V}^{*}}|f(x)|$ pour tout $x \in \mathbb{A}(V)$. La fonction

$$
\begin{aligned}
{[\cdot, \cdot]_{s}:\left(V^{*} \backslash\{0\}\right) \times(\mathbb{A}(V) \backslash\{0\}) } & \rightarrow \mathbb{R}_{+}, \\
(f, x) & \mapsto \frac{|f(x)|}{x_{\mathcal{V}^{*}}|f|_{\mathcal{V}^{*}}}
\end{aligned}
$$

ne dépend que de $s$ (et pas du choix du réseau $\mathcal{V}^{*}$ ), et se descend en une fonction

$$
[\cdot, \cdot]_{s}: P\left(V^{*}\right) \times \mathbb{P}(V) \rightarrow \mathbb{R}_{+}
$$

3.3.6. Lemme. - On a $\tau^{-1}\left(s^{*}\right)=\left\{x \in \mathbb{P}(V), \forall f \in P\left(V^{*}\right),[f, x]_{s}>q^{-1}\right\}$.

$4^{\mathrm{e}}$ SÉRIE - TOME $39-2006-\mathrm{N}^{\circ} 1$ 
Preuve. - (Indications.) Si $x \in \Omega(V)$, on a $\tau(x) \in s^{*}$ si et seulement si il existe $k \in \mathbb{N}$ et des sommets voisins de $s$ et deux à deux voisins et distincts $s_{1}, \ldots, s_{k}$ tels que $\tau(x)$ soit dans l'enveloppe convexe stricte (la facette) des points $s, s_{1}, \ldots, s_{k}$. En écrivant les normes des $s_{i}$ en coordonnées, on constatera sans difficultés que l'inégalité de l'énoncé est vérifiée.

Réciproquement, soit $x$ satisfaisant l'inégalité à droite du signe $=$ dans l'énoncé. On remarque tout d'abord que $x$ est nécessairement dans $\Omega(V)$, puis qu'on peut récupérer les $s_{i}$ par l'égalité

$$
\left\{s, s_{1}, \ldots, s_{k}\right\}=\left\{\text { sommets } t \text { tels que } \forall f \in P\left(V^{*}\right),[f, x]_{t}>q^{-1}\right\} .
$$

\subsubsection{Immeuble de Tits}

Notons $\mathfrak{X}^{(0)}$ l'ensemble de tous les sous- $K$-espaces vectoriels de $V^{*}$ propres (i.e. non nuls et différents de $V^{*}$ ) et $\mathfrak{X}:=\mathfrak{X}^{(0)} \cup\{0\}$. Ces ensembles sont partiellement ordonnés par l'inclusion et le complexe simplicial associé à $\mathfrak{X}^{(0)}$ est bien connu : il s'agit de l'immeuble de Tits de $P G L\left(V^{*}\right)$. Il a été muni dans [40] d'une topologie "pro-sphérique» pour laquelle l'action naturelle de $P G L\left(V^{*}\right)$ est continue. Pour $W \in \mathfrak{X}$ on définit

$$
W^{\perp}:=\{x \in \mathbb{P}(V), \forall f \in W,|f(x)|=0\} .
$$

C'est un sous-espace $K$-analytique projectif de $\mathbb{P}(V)$ et c'est $\mathbb{P}(V)$ tout entier si $W=0$. Pour un sommet $s$, on définit aussi

$$
\mathcal{H}_{s}(W):=\left\{x \in \mathbb{P}(V), \forall f \in W,[f, x]_{s} \leqslant q^{-1}\right\} .
$$

Si $W=0$ c'est encore $\mathbb{P}(V)$ et si $W \neq 0$, c'est un domaine analytique compact (au sens de Berkovich) auquel on peut penser comme à un certain «voisinage tubulaire» de $W^{\perp}$. Nous voulons aussi attacher à une facette $F$ des voisinages tubulaires $\mathcal{H}_{F}(W)$ de $W^{\perp}$. Pour cela, rappelons qu'une telle facette correspond à une suite infinie

$$
\left(\cdots \subset \mathcal{V}_{i}^{*} \subset \mathcal{V}_{i+1}^{*} \subset \cdots\right)_{i \in \mathbb{Z}}
$$

de $\mathcal{O}$-réseaux dans $V^{*}$ telle que pour tout $i \in \mathbb{Z}, \mathcal{V}_{i}^{*}=\varpi_{K} \mathcal{V}_{i+q+1}^{*}$ où $q$ est la dimension de $F$. Par ailleurs, un élément $x \in \mathbb{P}(V)$ est représenté par une semi-norme multiplicative $\tilde{x}$ sur $S\left(V^{*}\right)$. Notons $\kappa(x)$ le corps des fractions de l'anneau quotient de $S\left(V^{*}\right)$ par l'idéal $\left\{f \in S\left(V^{*}\right),|f(x)|=0\right\}$. Ce corps est muni d'une norme résiduelle et on a une factorisation :

$$
\tilde{x}: S\left(V^{*}\right) \rightarrow \kappa(x) \stackrel{||_{\kappa(x)}}{\longrightarrow} \mathbb{R}_{+}
$$

dont nous noterons encore $\tilde{x}$ la flèche de gauche. Nous posons alors

$$
\mathcal{H}_{F}(W):=\left\{x \in \mathbb{P}(V), \quad \forall i \in \mathbb{Z}, \tilde{x}\left(W \cap \mathcal{V}_{i+1}^{*}\right) \subseteq \tilde{x}\left(\mathcal{V}_{i}^{*}\right)\right\}
$$

le choix de $\tilde{x}$ au-dessus de $x$ étant clairement indifférent. Lorsque $F=s=\left(\cdots \subset \varpi_{K}^{-i} \mathcal{V}^{*} \subset\right.$ $\left.\varpi_{K}^{-i-1} \mathcal{V}^{*} \subset \cdots\right)$ est un sommet, la définition coïncide avec la précédente en vertu des équivalences

$$
\begin{aligned}
\forall i \in \mathbb{Z}, \tilde{x}\left(W \cap \mathcal{V}_{i+1}^{*}\right) \subseteq \tilde{x}\left(\mathcal{V}_{i}^{*}\right) & \Longleftrightarrow \tilde{x}\left(W \cap \mathcal{V}^{*}\right) \subseteq \varpi_{K} \tilde{x}\left(\mathcal{V}^{*}\right) \\
& \Longleftrightarrow \forall f \in W \text { t.q. }|f|_{\mathcal{V}^{*}}=1,|f(x)| \leqslant q^{-1} x_{\mathcal{V}^{*}}
\end{aligned}
$$

Par ailleurs, il est clair que si $F^{\prime} \subset F$, alors pour tout $W$, on a $\mathcal{H}_{F^{\prime}}(W) \subset \mathcal{H}_{F}(W)$. 
3.3.9. Proposition. - Notons $\mathfrak{X}_{F}$ l'ensemble de tous les fermés $\mathcal{H}_{F}(W)$ obtenus pour $W$ parcourant $\mathfrak{X}$.

(i) Pour toute facette $F, \mathfrak{X}_{F}$ est un arrangement dans $\mathbb{P}(V)$ dont l'ouvert complémentaire est $U_{F}=\tau^{-1}\left(F^{*}\right)$.

(ii) L'application $W \mapsto \mathcal{H}_{F}(W)$ est strictement croissante et induit pour tout $k$ une bijection:

$$
\mathfrak{X}^{(k)} / G_{F}^{+} \stackrel{\sim}{\longrightarrow} \mathfrak{X}_{F}^{(k)} .
$$

En particulier lorsque $F^{\prime} \subset F$, on obtient une application croissante $\mathfrak{X}_{F} \rightarrow \mathfrak{X}_{F^{\prime}}$ qui est un morphisme d'arrangement au-dessus de l'identité (cf. 3.3.2).

(iii) Pour tout $g \in P G L(V)$ et toute facette $F$, l'application $W \mapsto g^{*}(W)$ induit un isomorphisme d'arrangements $\mathfrak{X}_{F} \stackrel{\sim}{\sim} \mathfrak{X}_{g F}$ au-dessus de g.

(iv) Pour toute facette $F$ et tout $W \in \mathfrak{X}_{+}$, l'application canonique $H^{q}\left(\mathcal{H}_{F}(W)^{\mathrm{ca}}, \Lambda\right) \rightarrow$ $H^{q}\left(W^{\perp}{ }^{\text {ca }}, \Lambda\right)$ est un isomorphisme pour tout $q \geqslant 0$.

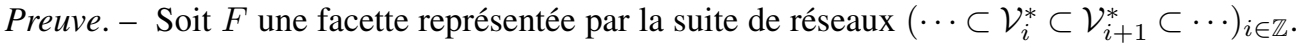
De la définition (3.3.8), on déduit que pour $W, W^{\prime} \in \mathfrak{X}^{(0)}$, on a

$$
\mathcal{H}_{F}(W)=\mathcal{H}_{F}\left(W^{\prime}\right) \quad \text { si et seulement si } \quad \forall i \in \mathbb{Z}, W \cap \mathcal{V}_{i}^{*}+\mathcal{V}_{i-1}^{*}=W^{\prime} \cap \mathcal{V}_{i}^{*}+\mathcal{V}_{i-1}^{*}
$$

Ceci a deux conséquences :

(i) Si $F^{\prime} \subset F$, on a $\mathcal{H}_{F^{\prime}}(W)=\mathcal{H}_{F^{\prime}}\left(W^{\prime}\right) \Longrightarrow \mathcal{H}_{F}(W)=\mathcal{H}_{F}\left(W^{\prime}\right)$, ou, en d'autres termes, l'application $W \mapsto \mathcal{H}_{F}(W)$ se factorise par l'application $W \mapsto \mathcal{H}_{F^{\prime}}(W)$.

(ii) D'après l'égalité $G_{F}^{+}=\left\{g \in G, \quad \forall i \in \mathbb{Z}, g \mathcal{V}_{i}^{*} \subseteq \mathcal{V}_{i}^{*}\right.$ et $\left.g_{\mid \mathcal{V}_{i}^{*}} / \mathcal{V}_{i-1}^{*}=\mathrm{Id}\right\}$, l'application $W \mapsto \mathcal{H}_{F}(W)$ se factorise par $\mathfrak{X} \rightarrow \mathfrak{X} / G_{F}^{+} \rightarrow \mathfrak{X}_{F}$. En particulier, $\mathfrak{X}_{F}$ est fini.

Pour $W, W^{\prime} \in \mathfrak{X}^{(0)}$, on veut maintenant trouver $W^{\prime \prime}$ tel que

$$
\mathcal{H}_{F}(W) \cap \mathcal{H}_{F}\left(W^{\prime}\right)=\mathcal{H}_{F}\left(W^{\prime \prime}\right) .
$$

Pour cela, le sous-espace $W^{\prime \prime} \subset V^{*}$ doit vérifier

$$
\forall i \in \mathbb{Z}, \quad W^{\prime \prime} \cap \mathcal{V}_{i}^{*}+\mathcal{V}_{i-1}^{*}=W \cap \mathcal{V}_{i}^{*}+W^{\prime} \cap \mathcal{V}_{i}^{*}+\mathcal{V}_{i-1}^{*}
$$

Il suffit bien sûr que l'égalité ci-dessus soit vérifiée pour $i \in\{0, \ldots, q+1\}$ où $q$ est la dimension de $F$. Plus généralement, on a :

3.3.10. Lemme. - Dans le contexte ci-dessus, soit $\left(\overline{W_{i}}\right)_{i=0, \ldots, q+1}$ une famille de sous- $k$ espaces vectoriels respectifs des espaces $\overline{V_{i}}:=\mathcal{V}_{i}^{*} / \mathcal{V}_{i-1}^{*}$. Alors il existe un sous-espace $W \subset V^{*}$ tel que pour tout $i$, l'image de $W \cap \mathcal{V}_{i}^{*}$ dans $\overline{V_{i}}$ soit $\overline{W_{i}}$.

La preuve de ce lemme se ramène à un simple exercice d'algèbre linéaire sur le $k$-espace vectoriel $\varpi^{-1} \mathcal{V}_{0}^{*} / \mathcal{V}_{0}^{*}$ que nous laissons au lecteur. Moyennant quoi, l'ensemble $\mathfrak{X}_{F}$ est bien un arrangement au sens de 3.3.2.

Dans le cas où $F=s$ est un sommet, la première définition que nous avons donnée de $\mathcal{H}_{s}(W)$ et le lemme 3.3.6 montrent que l'ouvert complémentaire de $\mathfrak{X}_{s}$ est bien $U_{s}=\tau^{-1}\left(s^{*}\right)$. Pour une facette générale, on a $\tau^{-1}\left(F^{*}\right)=\bigcap_{s \in F} \tau^{-1}\left(s^{*}\right)$ et les inclusions $\mathcal{H}_{s}(W) \subset \mathcal{H}_{F}(W)$ pour chaque sommet $s \in F$ montrent que l'ouvert complémentaire de $\mathfrak{X}_{F}$ est inclus dans $\tau^{-1}\left(F^{*}\right)$. Pour montrer l'autre inclusion, c'est un peu plus délicat; fixons $W \in \mathfrak{X}^{(0)}$ et convenons de noter $\overline{W_{i}}$ l'image de $W \cap \mathcal{V}_{i}^{*}$ dans $\overline{V_{i}}$. En appliquant le lemme précédent, on trouve des sous-espaces

$4^{e}$ SÉRIE - TOME $39-2006-\mathrm{N}^{\circ} 1$ 
$W_{i} \subset V^{*}$ tels que :

$$
\forall j \in \mathbb{Z}, \quad \text { l'image de } W_{i} \cap \mathcal{V}_{j}^{*} \text { dans } \quad \mathcal{V}_{j}^{*} / \mathcal{V}_{j-1}^{*}= \begin{cases}\overline{W_{j}} & \text { si } j=i \bmod q, \\ 0 & \text { si } j \neq i \bmod q\end{cases}
$$

On vérifie alors sur les définitions que $\mathcal{H}_{F}(W)=\bigcap_{i=0, \ldots, q+1} \mathcal{H}_{F}\left(W_{i}\right)$ et que d'autre part, si $s_{i}$ désigne le sommet de $F$ représenté par le réseau $\mathcal{V}_{i}^{*}$, alors

$$
\mathcal{H}_{F}\left(W_{i}\right)=\mathcal{H}_{s_{i-1}}\left(W_{i}\right) .
$$

Il s'ensuit que $\mathcal{H}_{F}(W)$ est inclus dans le complémentaire de $\tau^{-1}\left(F^{*}\right)$, et on obtient ainsi la deuxième inclusion cherchée. Enfin, la discussion précédente montre aussi que les $\mathcal{H}_{F}(W)$ sont des domaines analytiques compacts de $\mathbb{P}(V)$ (puisqu'on le sait pour les $\mathcal{H}_{s_{i-1}}\left(W_{i}\right)$ ) et la preuve du (i) est maintenant achevée.

Passons à (ii) : la croissance de $W \mapsto \mathcal{H}_{F}(W)$ est claire (rappelons que $\mathfrak{X}$ est ordonné par inclusion et $\mathfrak{X}_{F}$ par contenance!). Pour voir la stricte croissance, il suffit de remarquer que $\operatorname{dim}_{K}(W)=\sum_{i=0}^{q+1} \operatorname{dim}_{k}\left(\overline{W_{i}}\right)$ et de rappeler que $\mathcal{H}_{F}(W)=\mathcal{H}_{F}\left(W^{\prime}\right)$ si et seulement si $\overline{W_{i}}=\overline{W_{i}^{\prime}}$ pour tout $i \in \mathbb{Z}$. On a déja remarqué que la surjection $\mathfrak{X}^{(k)} \rightarrow \mathfrak{X}_{F}^{(k)}$ se factorise par $\mathfrak{X}^{(k)} \rightarrow \mathfrak{X}^{(k)} / G_{F}^{+}$. Le fait que l'application $\mathfrak{X}^{(k)} / G_{F}^{+} \rightarrow \mathfrak{X}_{F}^{(k)}$ est une bijection a été montré par Schneider-Stuhler [38, Corollary 6.5].

Le point (iii) est une conséquence évidente des définitions.

Pour la preuve de (iv), on s'inspire de l'idée de [38, Prop. 1.6] : traduite dans nos notations, elle consiste à exhiber une rétraction $p: \mathcal{H}_{F}(W) \rightarrow W^{\perp}$ de l'immersion $i: W^{\perp} \hookrightarrow \mathcal{H}_{F}(W)$ dont les fibres sont des polydisques affinoïdes. En effet, lorsqu'on a une telle rétraction et lorsque $\Lambda$ est de torsion (première à $p$ ), on considère les morphismes canoniques d'adjonction

$$
\Lambda_{W^{\perp}} \stackrel{\mathrm{can}}{\longrightarrow} R p_{*} p^{*} \Lambda_{W^{\perp}} \stackrel{\mathrm{can}}{\longrightarrow} R p_{*} i_{*} i^{*} p^{*} \Lambda_{W^{\perp}} \simeq \Lambda_{W^{\perp}} .
$$

L'isomorphisme de droite vient de l'égalité $p \circ i=\mathrm{Id}_{W^{\perp}}$ et la composée de ces deux morphismes est l'identité du faisceau $\Lambda_{W \perp}$. D'autre part, d'après Berkovich [2, 7.4.2] et notre hypothèse sur les fibres de $p$, la première flèche est un isomorphisme. La deuxième est donc aussi un isomorphisme et en appliquant le foncteur $R \Gamma\left(W^{\perp \mathrm{ca}}, \cdot\right)$ à cette deuxième flèche on obtient le résultat voulu, à savoir que l'application canonique de restriction $R \Gamma\left(\mathcal{H}_{F}(W)^{\mathrm{ca}}, \Lambda\right) \rightarrow$ $R \Gamma\left(W^{\perp \mathrm{ca}}, \Lambda\right)$ est un isomorphisme. Ceci règle le cas de torsion. Comme les espaces $\mathcal{H}_{F}(W)$ et $W^{\perp}$ sont compacts et quasi-algébriques, on déduit le cas $\lambda$-adique par passage à la limite grâce aux isomorphismes $H^{q}\left(X^{\mathrm{ca}}, \Lambda\right) \stackrel{\sim}{\longrightarrow} \varliminf_{\Perp} H^{q}\left(X^{\mathrm{ca}}, \Lambda / \lambda^{n}\right)$ valable pour de tels espaces.

Pour construire l'application $p$, on choisit des supplémentaires $\overline{Y_{i}}$ de $\overline{W_{i}}$ dans $\overline{V_{i}}$ pour $i=0, \ldots, q+1$ (avec les notations déja introduites plus haut) et on applique le lemme précédent pour trouver un sous-espace $Y \subset V^{*}$ tel que pour tout $i=0, \ldots, q+1$, l'image de $Y \cap \mathcal{V}_{i}^{*}$ dans $\overline{V_{i}}$ soit $\overline{Y_{i}}$. On remarque que $Y^{\perp} \cap W^{\perp}=\emptyset$ et $\operatorname{dim}\left(Y^{\perp}\right)+\operatorname{dim}\left(W^{\perp}\right)=d-2$, de sorte que l'on peut définir $p: \mathbb{P}(V) \backslash Y^{\perp} \rightarrow W^{\perp}$ la projection linéaire sur $W^{\perp}$ de centre $Y^{\perp}$. On a

$$
\begin{aligned}
Y^{\perp} \cap \mathcal{H}_{F}(W) & =\left\{x \in \mathbb{P}(V), \tilde{x}(Y)=0 \text { et } \forall i \in \mathbb{Z}, \tilde{x}\left(W \cap \mathcal{V}_{i}^{*}\right) \subseteq \tilde{x}\left(\mathcal{V}_{i-1}^{*}\right)\right\} \\
& \subseteq\left\{x \in \mathbb{P}(V), \forall i \in \mathbb{Z}, \tilde{x}\left(\mathcal{V}_{i}^{*}\right) \subseteq \tilde{x}\left(\mathcal{V}_{i-1}^{*}\right)\right\} \\
& =\emptyset,
\end{aligned}
$$

la dernière égalité venant de la non-nullité de $\tilde{x}$ associé à $x \in \mathbb{P}(V)$. Donc $p$ est bien définie sur $\mathcal{H}_{F}(W)$ et il est clair que c'est une rétraction de l'inclusion $i: W^{\perp} \hookrightarrow \mathcal{H}_{F}(W)$. 
On veut maintenant étudier les fibres de $p$ et de $p_{\mid \mathcal{H}_{F}(W)}$. Pour cela on fixe un élément $x$ de $W^{\perp} \subset \mathbb{P}(V)$. On note $\hat{\kappa}(x)$ la complétion du corps résiduel $\kappa(x)$ en $x$; la fibre de $p$ au-dessus de $x$ est naturellement munie d'une structure de $\hat{\kappa}(x)$-espace analytique affine. Pour expliciter cette structure, notons $V_{x}:=V \otimes_{K} \hat{\kappa}(x)$ et $\mathbb{P}\left(V_{x}\right)$ le $\hat{\kappa}(x)$-espace projectif associé. Notons aussi $V_{x}^{*}=$ $Y_{x} \oplus W_{x}$ la décomposition de $V_{x}^{*}:=V^{*} \otimes_{K} \hat{\kappa}(x)$ obtenue de la décomposition $V^{*}=Y \oplus W$ par extension des scalaires de $K$ à $\hat{\kappa}(x)$. On notera aussi $Y_{x}^{\perp}=\left\{z \in \mathbb{P}\left(V_{x}\right), \forall f \in Y_{x},|f(z)|=0\right\}$ le sous-espace projectif «othogonal» associé à $Y_{x}$; on a évidemment $Y_{x}^{\perp} \simeq Y^{\perp} \widehat{\otimes}_{K} \hat{\kappa}(x)$. Posons alors

$$
Y(x)=\left\{f \in Y_{x},|f(x)|=0\right\} \subseteq Y_{x} \subset V_{x}^{*} .
$$

(On voit ici $x$ comme un point de l'espace $\mathbb{P}\left(V_{x}\right)$.) La fibre de $p$ en $x$ munie de sa structure de $\hat{\kappa}(x)$-espace analytique est

$$
p^{-1}(x) \simeq Y(x)^{\perp} \backslash Y_{x}^{\perp} \subset \mathbb{P}\left(V_{x}\right) .
$$

C'est un espace affine dont l'algèbre de fonctions analytiques est la localisation de l'algèbre graduée $S\left(V_{x}^{*} / Y(x)\right)$ par un générateur, disons $f_{x}$, de $Y_{x} / Y(x)$. Ainsi l'application

$$
\begin{aligned}
\alpha: S\left(W_{x}\right) & \rightarrow S\left(V_{x}^{*} / Y(x)\right)_{f_{x}}, \\
f & \mapsto f f_{x}^{-1}
\end{aligned}
$$

induit un isomorphisme d'espaces $\hat{\kappa}(x)$-affines

$$
\alpha^{*}: p^{-1}(x) \stackrel{\sim}{\longrightarrow} \mathbb{A}\left(W_{x}\right)
$$

On veut maintenant calculer la fibre de $p_{\mid \mathcal{H}_{F}(W)}$ en $x$, c'est-à-dire l'image par l'isomorphisme $\alpha^{*}$ de l'intersection $p^{-1}(x) \cap \mathcal{H}_{F}(W)$.

La suite strictement croissante de $\mathcal{O}_{K}$-réseaux $\left(\mathcal{V}_{i}^{*}\right)_{i \in \mathbb{Z}}$ dans le $K$-espace $V^{*}$ représentant la facette $F$ induit par extension des scalaires puis passage au quotient une suite croissante de réseaux $\left(\mathcal{X}_{i}^{*}\right)_{i \in \mathbb{Z}}$ de $\mathcal{O}_{\hat{\kappa}(x)}$-réseaux dans le $\hat{\kappa}(x)$-espace $V_{x}^{*} / Y(x)$. Identifions $W_{x}$ avec son image dans $V_{x}^{*} / Y(x)$; notre choix du $K$-espace $Y$ nous assure que

$$
\forall i \in \mathbb{Z}, \quad \mathcal{X}_{i}^{*}=\left(\mathcal{X}_{i}^{*} \cap W_{x}\right) \oplus\left(\mathcal{X}_{i}^{*} \cap\left(Y_{x} / Y(x)\right)\right) .
$$

Remarquons qu'il existe un unique $i \in \mathbb{Z}$ tel que $f_{x} \in \mathcal{X}_{i}^{*} \cap\left(Y_{x} / Y(x)\right) \backslash \mathcal{X}_{i-1}^{*} \cap\left(Y_{x} / Y(x)\right)$. Nous supposerons, quitte à effectuer un décalage dans la numérotation des réseaux initiaux $\mathcal{V}_{i}^{*}$, que $i=-q-1$. Ceci permet d'écrire pour $i=1, \ldots, q+1$ :

$$
\mathcal{X}_{-i}^{*}=\left(W_{x} \cap \mathcal{X}_{-i}^{*}\right) \oplus \mathcal{O}_{\hat{\kappa}(x)} \cdot f_{x}
$$

et pour $i=0$,

$$
\mathcal{X}_{0}^{*}=\left(W_{x} \cap \mathcal{X}_{0}^{*}\right) \oplus \mathcal{O}_{\hat{\kappa}(x)} \cdot \varpi_{K}^{-1} f_{x} .
$$

Maintenant, nous pouvons choisir une $\mathcal{O}_{\hat{\kappa}(x)}$-décomposition

$$
W_{x} \cap \mathcal{X}_{0}^{*}=\mathcal{L}^{0} \oplus \cdots \oplus \mathcal{L}^{q}
$$

avec éventuellement $\mathcal{L}^{i}=0$ pour certains $i$ mais telle que

$4^{\mathrm{e}}$ SÉRIE - TOME $39-2006-\mathrm{N}^{\circ} 1$ 


$$
\begin{aligned}
W_{x} \cap \mathcal{X}_{-1}^{*} & =\varpi_{K} \mathcal{L}^{0} \oplus \mathcal{L}^{1} \oplus \cdots \oplus \mathcal{L}^{q}, \\
& \vdots \\
W_{x} \cap \mathcal{X}_{-q}^{*} & =\varpi_{K} \mathcal{L}^{0} \oplus \cdots \oplus \varpi_{K} \mathcal{L}^{q-1} \oplus \mathcal{L}^{q} .
\end{aligned}
$$

Pour un sous- $\mathcal{O}_{\hat{\kappa}(x)}$-module $\mathcal{X}$ de type fini de $V_{x}^{*} / Y(x)$ et un élément $y \in p^{-1}(x)$ vu comme une norme sur l'algèbre $S\left(V_{x}^{*} / Y(x)\right)$, notons

$$
y(\mathcal{X}):=\sup _{f \in \mathcal{X}}|f(y)| .
$$

Alors la condition pour que $y \in \mathcal{H}_{F}(W)$ s'écrit

$$
\left(\forall i \in\{0, \ldots, q\}, \quad y\left(W_{x} \cap \mathcal{X}_{-i}\right) \leqslant y\left(\mathcal{X}_{-i-1}\right)\right)
$$

ou encore

$$
\left\{\begin{array}{l}
\sup \left(y\left(\mathcal{L}^{0}\right), \ldots, y\left(\mathcal{L}^{q}\right)\right) \leqslant \sup \left(q_{K}^{-1} y\left(\mathcal{L}^{0}\right), y\left(\mathcal{L}^{1}\right), \ldots, y\left(\mathcal{L}^{q}\right),\left|f_{x}(y)\right|\right) \\
\sup \left(q_{K}^{-1} y\left(\mathcal{L}^{0}\right), \ldots, y\left(\mathcal{L}^{q}\right)\right) \leqslant \sup \left(q_{K}^{-1} y\left(\mathcal{L}^{0}\right), q_{K}^{-1} y\left(\mathcal{L}^{1}\right), y\left(\mathcal{L}^{2}\right), \ldots,\left|f_{x}(y)\right|\right) \\
\quad \vdots \\
\sup \left(q_{K}^{-1} y\left(\mathcal{L}^{0}\right), \ldots, q_{K}^{-1} y\left(\mathcal{L}^{q-1}\right), y\left(\mathcal{L}^{q}\right)\right) \leqslant \sup \left(q_{K}^{-1} y\left(\mathcal{L}^{0}\right), \ldots, q_{K}^{-1} y\left(\mathcal{L}^{q}\right),\left|f_{x}(y)\right|\right)
\end{array}\right.
$$

Nous allons montrer que ce système d'inégalités est équivalent à la seule inégalité

$$
\sup \left(y\left(\mathcal{L}^{0}\right), \ldots, y\left(\mathcal{L}^{q}\right)\right) \leqslant\left|f_{x}(y)\right| .
$$

Tout d'abord, il est clair que si (3.3.11) est vérifiée alors le système d'inégalités ci-dessus l'est aussi. Réciproquement, supposons le système d'inégalités vérifié et notons $k \in\{0, \ldots, q\}$ le plus grand indice tel que $y\left(\mathcal{L}^{k}\right)=\sup _{i=0, \ldots, q}\left(y\left(\mathcal{L}^{i}\right)\right)$. Alors la $(k+1)$-ième ligne du système nous dit que $y\left(\mathcal{L}^{k}\right) \leqslant \sup \left(q_{K}^{-1} y\left(\mathcal{L}^{0}\right), \ldots, q_{K}^{-1} y\left(\mathcal{L}^{k}\right), y\left(\mathcal{L}^{k+1}\right), \ldots, y\left(\mathcal{L}^{q}\right),\left|f_{x}(y)\right|\right)$. Mais le choix de $k$ entraîne alors que $y\left(\mathcal{L}^{k}\right) \leqslant\left|f_{x}(y)\right|$.

On en déduit que l'isomorphisme $\alpha^{*}$ envoie $p^{-1}(x) \cap \mathcal{H}_{F}(W)$ sur le polydisque $D_{\mathcal{X}_{0}}$ de $\mathbb{A}\left(W_{x}\right)$ défini par

$$
D_{\mathcal{X}_{0}}:=\left\{z \in \mathbb{A}\left(W_{x}\right), \sup _{f \in W_{x} \cap \mathcal{X}_{0}^{*}}|f(z)| \leqslant 1\right\} .
$$

\subsubsection{Le système de suites spectrales $\mathbb{E}_{r}^{p q}$}

D'après la proposition précédente, on a construit un système de coefficients $F \mapsto \mathfrak{X}_{F}$, $G$-équivariant sur $B T$ en arrangements (i.e. à valeurs dans la catégorie $\operatorname{Arr}(\mathbb{P}(V))$ définie en 3.3.2). Par fonctorialité, on en déduit plusieurs autres systèmes de coefficients $G$-équivariants comme

- un système en suites spectrales $\mathbb{E}_{r}^{p q}: F \mapsto E_{r}^{p q}\left(\mathfrak{X}_{F}\right)$ (cf. 3.3.4) dont le $\mathbb{E}_{1}$ est donné par

- des systèmes en complexes de $\Lambda W_{K}$-modules $\mathbb{E}_{1}^{p *}: F \mapsto \mathcal{C}^{*}\left(\mathfrak{X}_{F}, X \mapsto H^{p}\left(X^{\text {ca }}, \Lambda\right)\right)$, et le système des aboutissements est un gradué,

- des systèmes $F \mapsto H_{c}^{p+q}\left(U_{F}^{\mathrm{ca}}, \Lambda\right)$.

Introduisons maintenant le sous-ensemble

$$
\mathfrak{X}(p):=\left\{W \in \mathfrak{X}, H^{p}\left(W^{\perp}, \Lambda\right) \neq 0\right\} .
$$


C'est un sous-ensemble partiellement ordonné plein de $\mathfrak{X}$ qui est non-vide seulement si $p$ est pair et $\leqslant 2 d-2$. Dans ce cas, l'ensemble simplicial associé est profini de dimension $d-1-p / 2$, et a été étudié par Schneider et Stuhler [38, prop. 3.6, proof]. Le dictionnaire avec leurs notations est le suivant : $\mathfrak{X}(p)^{(k)}$ s'écrirait avec leurs notations $\mathcal{N} \mathcal{T}_{k}^{(d-1-p / 2)}$. Nous renvoyons à loc. cit, notamment avant leur lemme 3.3, pour la définition précise de la topologie sur $\mathfrak{X}(p)^{(k)}$ et nous noterons $C^{\infty}\left(\mathfrak{X}(p)^{(k)}, \Lambda\right)$ le $\Lambda$-module des fonctions localement constantes $\mathfrak{X}(p)^{(k)} \rightarrow \Lambda$. Suivant Schneider et Stuhler, on associe à $\mathfrak{X}(p)$ le complexe de cochaînes continues $\left(\mathcal{C}^{*}(\mathfrak{X}(p), \Lambda), \partial^{*}\right)$ suivant :

$$
\begin{aligned}
\Lambda & \stackrel{\partial^{0}}{\longrightarrow} C^{\infty}\left(\mathfrak{X}(p)^{(0)}, \Lambda\right) \stackrel{\partial^{1}}{\longrightarrow} \cdots \stackrel{\partial^{k}}{\longrightarrow} C^{\infty}\left(\mathfrak{X}(p)^{(k)}, \Lambda\right) \stackrel{\partial^{k+1}}{\longrightarrow} \cdots \\
& \stackrel{\partial^{d-1-p / 2}}{\longrightarrow} C^{\infty}\left(\mathfrak{X}(p)^{(d-1-p / 2)}, \Lambda\right)
\end{aligned}
$$

où la différentielle est définie comme la somme alternée des applications de dégénéréscence (version continue de 3.3.1). Ce complexe est naturellement un complexe de $\Lambda G$-modules lisses admissibles.

3.3.13. Proposition. - Il existe pour tout $p \in \mathbb{N}$ pair et $\leqslant 2 d-2$ un isomorphisme de systèmes de coefficients $G$-équivariants en complexes de $\Lambda W_{K}$-modules :

$$
\mathbb{E}_{1}^{p *} \stackrel{\sim}{\longrightarrow}\left(F \mapsto \mathcal{C}^{*}(\mathfrak{X}(p), \Lambda)^{G_{F}^{+}}(-p / 2)\right) .
$$

Pour p impair, $\mathbb{E}_{1}^{p *}=0$.

Preuve. - Commençons par souligner les conséquences suivantes du point (iv) de la proposition 3.3.9:

(i) La cohomologie de $\mathcal{H}_{F}(W)$ est donnée par

$$
H^{p}\left(\mathcal{H}_{F}(W)^{\mathrm{ca}}, \Lambda\right) \simeq \begin{cases}\Lambda(-p / 2) & \text { si } p \leqslant 2 c(W) \text { est pair, } \\ 0 & \text { sinon, }\end{cases}
$$

où $c(W)$ désigne la codimension du sous-espace $W$ de $V^{*}$.

(ii) Pour toute facette $F$ et tous $W, W^{\prime}$ tels que $\mathcal{H}_{F}\left(W^{\prime}\right) \subset \mathcal{H}_{F}(W)$, l'application

$$
H^{p}\left(\mathcal{H}_{F}(W)^{\mathrm{ca}}, \Lambda\right) \rightarrow H^{p}\left(\mathcal{H}_{F}\left(W^{\prime}\right)^{\mathrm{ca}}, \Lambda\right)
$$

est un isomorphisme pour tout $p \leqslant 2 c\left(W^{\prime}\right)$.

(iii) Pour tout $W \in \mathfrak{X}^{(0)}$ et toutes facettes $F^{\prime} \subset F$, l'application canonique

$$
H^{p}\left(\mathcal{H}_{F}(W)^{\mathrm{ca}}, \Lambda\right) \rightarrow H^{p}\left(\mathcal{H}_{F^{\prime}}(W)^{\mathrm{ca}}, \Lambda\right)
$$

est un isomorphisme pour tout $p$.

Fixons maintenant $F$ et introduisons le sous-ensemble

$$
\mathfrak{X}_{F}(p)=\left\{X \in \mathfrak{X}_{F}, H^{p}\left(X^{\mathrm{ca}}, \Lambda\right) \neq 0\right\} .
$$

C'est un sous-ensemble partiellement ordonné de $\mathfrak{X}_{F}$ tel que $X \leqslant X^{\prime} \in \mathfrak{X}_{F} \Longrightarrow X \in \mathfrak{X}_{F}$ (par (ii) ci-dessus) et qui est non vide seulement si $p=2 i$ est pair et $\leqslant 2 d-2$ (par (i)). Toujours d'après (i) et (ii) ci-dessus, le système de coefficients $X \mapsto H^{p}\left(X^{\mathrm{ca}}, \Lambda\right)$ sur $\mathfrak{X}_{F}$ est supporté par $\mathfrak{X}_{F}(p)$ et y est constant. On a donc une identité de complexes de cochaînes

$$
\mathcal{C}^{*}\left(\mathfrak{X}_{F}(p), \Lambda\right)(-p / 2) \stackrel{\sim}{\longrightarrow} \mathcal{C}^{*}\left(\mathfrak{X}_{F}, X \mapsto H^{p}\left(X^{\mathrm{ca}}, \Lambda\right)\right)
$$

$4^{\text {e }}$ SÉRIE - TOME $39-2006-\mathrm{N}^{\circ} 1$ 
où $\Lambda$ dans le terme de gauche désigne le système de coefficients sur $\mathfrak{X}_{F}(p)$ constant $X \mapsto \Lambda$.

De plus, par (iii) l'application $\mathfrak{X}_{F^{\prime}} \rightarrow \mathfrak{X}_{F}$ pour $F^{\prime} \subset F$ envoie $\mathfrak{X}_{F^{\prime}}(p)$ dans $\mathfrak{X}_{F}(p)$ et pour tout $g \in G, \mathfrak{X}_{g(F)}(p)=g\left(\mathfrak{X}_{F}(p)\right)$. En résumé, le terme $\mathbb{E}_{1}$ est donné en tant que système de coefficients $G$-équivariant en $\Lambda W_{K}$-modules par

$$
\mathbb{E}_{1}^{p *}=\left(F \mapsto \mathcal{C}^{*}\left(\mathfrak{X}_{F}(p), \Lambda\right)(-p / 2)\right) .
$$

Il résulte maintenant de 3.3.9(ii) que pour tout $k \geqslant 0$ et toute facette $F$, l'application canonique $\mathfrak{X} \rightarrow \mathfrak{X}_{F}$ induit une bijection $\mathfrak{X}(p)^{(k)} / G_{F}^{+} \stackrel{\sim}{\longrightarrow} \mathfrak{X}_{F}(p)^{(k)}$. De cette bijection on tire un isomorphisme de complexes de $\Lambda$-modules :

$$
\mathcal{C}^{*}\left(\mathfrak{X}_{F}(p), \Lambda\right) \stackrel{\sim}{\longrightarrow} \mathcal{C}^{*}(\mathfrak{X}(p), \Lambda)^{G_{F}^{+}} .
$$

Par construction les isomorphismes ainsi obtenus sont compatibles à l'action de $G$ et aux inclusions de facettes : ce sont des isomorphismes de systèmes de coefficients $G$-équivariants sur $B T$ à valeurs dans les complexes de $\Lambda$-modules. On a donc terminé la preuve.

3.3.14. Corollaire. - Le terme $\mathbb{E}_{2}$ du système de suites spectrales 3.3 .12 est donné par

$$
\mathbb{E}_{2}^{p q} \simeq \begin{cases}F \mapsto\left(\pi_{\{1, \ldots, p / 2\}}^{\Lambda}\right)^{G_{F}^{+}}(-p / 2) & \text { si } p \leqslant 2 d-2 \text { est pair et } q=d-1-p / 2, \\ 0 & \text { sinon. }\end{cases}
$$

Preuve. - D'après Schneider et Stuhler [38, lemma 4.1], la cohomologie du complexe $\mathcal{C}^{*}(\mathfrak{X}(p), \Lambda)$ est concentrée en degré $d-1-p / 2$ et est isomorphe à $\pi_{\{1, \ldots, p / 2\}}^{\Lambda}(-p / 2)$.

\subsubsection{Fin de la preuve de la proposition 3.2.6}

D'après le corollaire précédent, le système de suites spectrales (ou si l'on préfère, la suite spectrale en systèmes de coefficients) $\mathbb{E}_{r}^{p q}$ dégénère en $\mathbb{E}_{2}$ et fournit des isomorphismes

$$
\mathbb{E}_{2}^{2 i, d-1-i} \stackrel{\sim}{\longrightarrow}\left(F \mapsto H^{d-1+i}\left(U_{F}^{\mathrm{ca}}, \Lambda\right)\right) .
$$

Précisons de manière informelle ce que les isomorphismes du théorème 3.1.1 ont de canonique. La description de la cohomologie de $\mathbb{P}^{d}$ et des morphismes de restriction de celle-ci à celle des sous-variétés linéaire est canonique. Les suites spectrales que nous utilisons dégénèrent toutes fortement en des isomorphismes. Pour les obtenir, il y a des choix d'orientations sur les nerfs des recouvrements utilisés. Dans le cas des $\mathfrak{X}_{F}$, on manipule des posets et il y a un choix d'orientation «naturel» que nous avons fait. Dans le cas de $B T$, l'utilisation des facettes orientées règle le problème des signes. Il reste que la définition des $\pi_{I}^{\Lambda}$ dépend du choix de la paire de Borel $(B, T)$, mais il en dépend canoniquement!

\section{Complexe de cohomologie et représentations galoisiennes}

Ici encore, l'anneau $\Lambda$ est une $\mathbb{Z}_{l}$-algèbre locale que l'on supposera soit de type fini en tant que $\mathbb{Z}_{l}$-module, soit extension algébrique de $\mathbb{Q}_{l}$.

\section{1. Énoncé du théorème}

On rappelle qu'on a fixé une paire de Borel $(B, T)$ dans $G L_{d}(K)$ et numéroté l'ensemble des racines $S$ correspondant au-dessus du théorème 3.1.1 qui décrit la cohomologie de $\Omega_{K}^{d-1}$. 


\subsubsection{Représentations galoisiennes associées aux $\pi_{I}^{\Lambda}$}

Soit $V$ le $\Lambda$-module $\Lambda \oplus \Lambda(1) \oplus \cdots \oplus \Lambda(d-1)$. Il est muni de l'action $\tau_{s s}^{\Lambda}$ de $W_{K}$, somme directe des caractères $w \mapsto|w|^{i}$. Lorsque $\Lambda$ est banal pour $G L_{d}(K)$, nous allons définir des représentations $\tau_{I}^{\Lambda}$ de $W_{K}$ sur $V$.

Soit $\left(E_{i j}\right)_{0 \leqslant i, j \leqslant d-1}$ la base canonique de l'algèbre $\mathcal{M}_{d}(\Lambda)$ des matrices $d \times d$ sur $\Lambda$. Pour chaque $I \subseteq S$, on note $N_{I}$ la matrice nilpotente

$$
N_{I}:=\sum_{i \in I^{c}} E_{i, i-1}
$$

où $I^{c}:=S \backslash I$ désigne le complémentaire de $I$ dans $S$. La matrice $N_{I}$ définit un morphisme $V \rightarrow V(-1)$. Si $\mu \in \mathbb{Z}_{l}(1)$, l'endomorphisme $N_{I} \otimes \mu$ de $V$ est nilpotent d'ordre $\leqslant d$ et on peut donc prendre son exponentielle puisque $d$ ! est inversible dans $\Lambda$ supposé banal.

Choisissons alors un relèvement de Frobenius géométrique $\phi$, ce qui nous permet d'écrire tout $w \in W_{K}$ sous la forme unique $w=\phi^{\nu(w)} i_{\phi}(w)$ où $\nu(w) \in \mathbb{Z}$ et $i_{\phi}(w) \in I_{K}$, et notons $t_{l}: I_{K} \rightarrow \mathbb{Z}_{l}(1)$ la projection vers le $l$-quotient de $I_{K}$. Un calcul rapide montre que pour chaque $I \subseteq S$, la formule

$$
w \in W_{K} \mapsto \tau_{I}^{\Lambda, \phi}(w):=\tau_{s s}^{\Lambda}(w) \cdot \exp \left(N_{I} \otimes t_{l}\left(i_{\phi}(w)\right)\right) \in \operatorname{End}_{\Lambda}(V)
$$

définit une représentation de $W_{K}$ sur $V$. Comme la notation le suggère, celle-ci dépend du choix de $\phi$ mais un autre calcul (un peu moins rapide, cf. [16, 8.4.2]) montre que sa classe n'en dépend pas. Nous noterons donc simplement $\tau_{I}^{\Lambda}$ cette classe.

Lorsque $I=\emptyset$, la représentation $\tau_{\emptyset}^{\Lambda}$ est donc la représentation «spéciale $S p_{d}^{\Lambda}$ de dimension $d$ de $W_{K}$. Avec un peu de combinatoire, on peut exprimer toutes les $\tau_{I}^{\Lambda}$ en fonction des représentations spéciales de dimension plus petite que $d$. Pour cela, munissons l'ensemble $\{0, \ldots, d-1\}$ d'une structure de graphe orienté en déclarant que $x \rightarrow y$ si et seulement si $(x \leqslant y$ et $\left.\{x+1, \ldots, y\} \subset I^{c}\right)$. En particulier, on a $x \rightarrow x$ pour tout $x$. La partition de $\{0, \ldots, d-1\}$ en composantes connexes s'écrit

$$
\left\{0, \ldots, i_{1}-1\right\} \sqcup\left\{i_{1}, \ldots, i_{2}-1\right\} \sqcup \cdots \sqcup\left\{i_{|I|}, \ldots, d-1\right\}
$$

où $I=\left\{i_{1}, \ldots, i_{|I|}\right\} \subset\{1, \ldots, d-1\}$. Convenons de poser $i_{0}:=0$ et $i_{|I|+1}:=d$. Alors la longueur de la $k$-ième composante connexe est $d_{k}:=i_{k+1}-i_{k}$ où $0 \leqslant k \leqslant|I|$. La somme des $d_{k}$ est égale à $d$ et on retrouve les $i_{k}$ par la formule $i_{k}=d_{0}+\cdots+d_{k-1}$. Ceci étant, on vérifie la formule élémentaire suivante :

$$
\tau_{I}^{\Lambda} \simeq \bigoplus_{k=0}^{|I|} S p_{d_{k}}^{\Lambda}\left(i_{k}\right)
$$

4.1.2. FAIT. - Lorsque $\Lambda=\overline{\mathbb{Q}}_{l}$, resp. $\Lambda=\overline{\mathbb{F}}_{l}$ avec l banal pour $G L_{d}(K)$, la classe de représentations $\sigma_{d}\left(\pi_{I}\right):=\tau_{I} \otimes|-|^{\frac{1-d}{2}}$ est celle associée à $\pi_{I}$ par la correspondance de Langlands, resp. de Langlands-Vignéras.

Preuve. - Donnons tout d'abord quelques explications sur l'énoncé. La correspondance de Langlands n'est «bien définie» que pour $\Lambda=\mathbb{C}$. Il se trouve que la variante «de Hecke» $\pi \mapsto \sigma_{d}(\pi) \otimes|-|^{\frac{d-1}{2}}$ est invariante par l'action naturelle des automorphismes de $\mathbb{C}$ et se transporte donc à $\overline{\mathbb{Q}}_{l}$ sans ambiguïté [25]. Par contre, pour obtenir une correspondance de Langlands sur $\overline{\mathbb{Q}}_{l}$, il faut choisir une racine de $p$. De même pour la correspondance de LanglandsVignéras sur $\overline{\mathbb{F}}_{l}$. Nous supposerons implicitement qu'un tel choix a été effectué. Tout cela est bien

4 SÉRIE - TOME $39-2006-\mathrm{N}^{\circ} 1$ 
connu des spécialistes; nous omettons ici les explications car nous ne considérons que des cas particulièrement simples de ces correspondances.

Supposons $\Lambda=\overline{\mathbb{Q}}_{l}$. Pour déterminer la correspondante de Langlands de $\pi_{I}$, il faut calculer ses paramètres de Langlands. Ceci a été fait dans la remarque 2.3.4 : on y obtient que $\pi_{I}$ est l'unique quotient irréductible de l'induite $i_{P_{-w_{0}\left(I^{c}\right)}^{G}}^{G}\left(\delta_{P_{-w_{0}\left(I^{c}\right)}}^{1 / 2} S t_{M_{-w_{0}\left(I^{c}\right)}}\right)$, où $w_{0}$ désigne l'élément de plus grande longueur de $W=\mathfrak{S}_{d}$. Dans le cas présent, on a simplement $-w_{0}(s)=d-s$ pour tout $s \in S=\{1, \ldots, d-1\}$.

Pour traduire ceci en termes de produits à la Zelevinski, on utilise les notations précédentes $I=\left\{i_{1}, \ldots, i_{|I|}\right\}$ et $d_{k}:=i_{k+1}-i_{k}$. Le sous-groupe de Levi $M_{-w_{0}\left(I^{c}\right)}$ de $G L_{d}(K)$ s'identifie à $G L_{d_{|I|}}(K) \times \cdots \times G L_{d_{0}}(K)$ et un calcul élémentaire du module de $P_{-w_{0}\left(I^{c}\right)}$ montre que

$$
i_{M_{-w_{0}\left(I^{c}\right)}^{G_{d}}}\left(\delta_{P_{-w_{0}\left(I^{c}\right)}}^{1 / 2} S t_{M_{-w_{0}\left(I^{c}\right)}}\right)=|\operatorname{det}|^{n_{|I|}} S t_{G_{d_{|I|}}} \times \cdots \times|\operatorname{det}|^{n_{0}} S t_{G_{d_{0}}}
$$

en posant pour tout $k=0, \ldots,|I|$,

$$
\begin{aligned}
n_{k} & :=\left(-d_{|I|}-\cdots-d_{k+1}+d_{k-1}+\cdots+d_{0}\right) / 2 \\
& =i_{k}+\left(d_{k}-d\right) / 2 .
\end{aligned}
$$

On en déduit la formule suivante pour $\sigma_{d}\left(\pi_{I}\right)$, cf. [26, 2.9]

$$
\sigma_{d}\left(\pi_{I}\right)=|-|^{n|I|} \sigma_{d_{|I|}}\left(S t_{G_{d_{|I|}}}\right) \oplus \cdots \oplus|-|^{n_{0}} \sigma_{d_{0}}\left(S t_{G_{d_{0}}}\right)
$$

et on rappelle que pour tout $k$, on a avec nos conventions

$$
\sigma_{d_{k}}\left(S t_{G_{d_{k}}}\right)=S p_{d_{k}} \otimes|-|^{\frac{1-d_{k}}{2}} .
$$

On en conclut que $\sigma_{d}\left(\pi_{I}\right)=|-|^{(1-d) / 2} \tau_{I}^{\overline{\mathbb{Q}}_{l}}$. On déduit le cas $\overline{\mathbb{F}}_{l}$ par réduction modulo $l$, car $l$ est supposé banal.

\subsubsection{Le complexe de cohomologie}

Notons $P G_{d}:=P G L_{d}(K)$. L'action de $G_{d}$ sur $\Omega_{K}^{d-1 \text {,ca }}$ se factorise par $P G_{d}$. Notons aussi $D^{b}\left(\Lambda P G_{d}\right)$ la catégorie dérivée de la catégorie abélienne des $\Lambda$-représentations lisses de $P G_{d}$. Les résultats de l'appendice $\mathrm{B}$, cf. notamment B.2.6, permettent de définir un complexe

$$
R \Gamma_{c}\left(\Omega_{K}^{d-1, \mathrm{ca}}, \Lambda\right) \in D^{b}\left(\Lambda P G_{d}\right)
$$

muni d'une action de $W_{K}$, et dont les modules de cohomologie s'identifient canoniquement aux modules $H_{c}^{i}\left(\Omega_{K}^{d-1, \text { ca }}, \Lambda\right)$. Une propriété importante de ce complexe est la suivante : supposons que $\Gamma \subset P G_{d}$ soit un sous-groupe discret et sans torsion de $P G_{d}$. On sait alors que $\Gamma$ agit librement sur $\Omega_{K}^{d-1}$ et qu'on peut munir $\Omega_{K}^{d-1} / \Gamma$ d'une structure de $K$-espace analytique telle que le quotient $\Omega_{K}^{d-1} \rightarrow \Omega_{K}^{d-1} / \Gamma$ soit un revêtement analytique galoisien. Dans ces conditions, la proposition B.3.1 montre l'existence d'un isomorphisme $W_{K}$-équivariant dans $D^{b}(\Lambda)$

$$
\Lambda \otimes_{\Lambda[\Gamma]}^{L} R \Gamma_{c}\left(\Omega_{K}^{d-1, \mathrm{ca}}, \Lambda\right) \stackrel{\sim}{\longrightarrow} R \Gamma_{c}\left(\Omega_{K}^{d-1, \mathrm{ca}} / \Gamma, \Lambda\right)
$$

où nous avons fait l'abus de noter encore $R \Gamma_{c}\left(\Omega_{K}^{d-1, \text { ca }}, \Lambda\right) \in D^{b}(\Lambda \Gamma)$ l'image du complexe précédemment défini par le foncteur «d'oubli» $D^{b}\left(\Lambda P G_{d}\right) \rightarrow D^{b}(\Lambda \Gamma)$, où le produit tensoriel 
est pris pour l'augmentation $\Lambda[\Gamma] \rightarrow \Lambda$, et où nous avons noté $R \Gamma_{c}\left(\Omega_{K}^{d-1, \mathrm{ca}} / \Gamma, \Lambda\right) \in D^{b}(\Lambda)$ le complexe de cohomologie de l'espace $\Omega_{K}^{d-1, \mathrm{ca}} / \Gamma$.

Comme dans l'introduction, on a le foncteur

$$
\begin{aligned}
\mathcal{H}^{*}: D^{b}(\Lambda) & \rightarrow\{\Lambda \text {-modules }\}, \\
\mathcal{C}^{\bullet} & \mapsto \bigoplus_{i \in \mathbb{Z}} \mathcal{H}^{i}\left(\mathcal{C}^{\bullet}\right) .
\end{aligned}
$$

Le théorème principal de ce travail est le suivant :

4.1.5. THÉORÈME. - Supposons $\Lambda$ fortement banal pour $G L_{d}(K)$, au sens de 2.1.6. Pour tout $I \subseteq S$, il existe un isomorphisme $W_{K}$-équivariant dans $D^{b}(\Lambda)$

$$
R \operatorname{Hom}_{D^{b}\left(\Lambda P G_{d}\right)}\left(R \Gamma_{c}\left(\Omega_{K}^{d-1, \mathrm{ca}}, \Lambda\right), \pi_{I}^{\Lambda}\right)[1-d] \stackrel{\sim}{\longrightarrow} \bigoplus_{k=0}^{|I|} S p_{d_{k}}\left(i_{k}\right)[-|I|+2 k]
$$

dont on déduit un isomorphisme de $\Lambda W_{K}$-modules

$$
\mathcal{H}^{*}\left(R \operatorname{Hom}_{D^{b}\left(\Lambda P G_{d}\right)}\left(R \Gamma_{c}\left(\Omega_{K}^{d-1, \mathrm{ca}}, \Lambda\right), \pi_{I}^{\Lambda}\right)\right) \stackrel{\sim}{\longrightarrow} \tau_{I}^{\Lambda} .
$$

On en déduit le théorème 1.1 grâce à 4.1 .2 ci-dessus.

\subsection{Action de $W_{K}$ sur le complexe de cohomologie}

Dans cette section, on explicite le morphisme $\gamma: W_{K} \rightarrow \operatorname{End}_{D^{b}\left(P G_{d}\right)}\left(R \Gamma_{c}\left(\Omega_{K}^{d-1, \text { ca }}, \Lambda\right)\right)$ qui définit l'action de $W_{K}$ sur $R \Gamma_{c}\left(\Omega_{K}^{d-1, \text { ca }}, \Lambda\right)$.

Commençons par la remarque suivante concernant la restriction de $\gamma$ à $I_{K}$ :

4.2.1. Lemme. - La restriction $\gamma_{\mid I_{K}}$ se factorise par le l-quotient $I_{K} \stackrel{t_{l}}{\longrightarrow} \mathbb{Z}_{l}(1)$. En particulier, si $d$ ! est inversible dans $\Lambda$ (par exemple si $\Lambda$ est banal), il existe un unique morphisme $W_{K}$-équivariant

$$
N: R \Gamma_{c}\left(\Omega_{K}^{d-1, \mathrm{ca}}, \Lambda\right) \rightarrow R \Gamma_{c}\left(\Omega_{K}^{d-1, \mathrm{ca}}, \Lambda\right)(-1)
$$

«nilpotent $»$ d'ordre $\leqslant$ d et tel que

$$
\forall i \in I_{K}, \quad \gamma(i)=\exp \left(N \otimes t_{l}(i)\right) .
$$

Preuve. - D'après la description 3.1.1, l'inertie agit trivialement sur les objets de cohomologie de $R \Gamma_{c}$. On a donc $\gamma\left(I_{K}\right) \subset \mathcal{U}:=1+\mathcal{N}$ où $\mathcal{N}$ désigne le noyau du morphisme canonique de $\Lambda$-algèbres

$$
\operatorname{End}_{D^{b}\left(P G_{d}\right)}\left(R \Gamma_{c}\left(\Omega_{K}^{d-1, \mathrm{ca}}, \Lambda\right)\right) \stackrel{\mathrm{can}}{\longrightarrow} \prod_{i=0}^{d-1} \operatorname{End}_{P G_{d}}\left(H_{c}^{d-1+i}\left(\Omega_{K}^{d-1, \mathrm{ca}}, \Lambda\right)\right) .
$$

Le point (i) du lemme A.1.4 montre dans un contexte général que $\mathcal{N}$ est formé d'éléments nilpotents d'ordre $\leqslant d$.

Supposons que $\Lambda=\mathbb{Z}_{l}$. D'après le résultat général A.2.1, le groupe $\mathcal{U}$ est un pro-l-groupe, pourvu que $\operatorname{Ext}_{\mathbb{Z}_{l} P G_{d}}^{q-p}\left(H_{c}^{q}, H_{c}^{p}\right)$ soit un $\mathbb{Z}_{l}$-module de type fini pour tous $p, q \in \mathbb{Z}$. Or, par [38, Thm. 6.8], on sait que les $H_{c}^{q}$ admettent des résolutions de longueur finie par des représentations

$4^{\text {e }}$ SÉRIE - TOME $39-2006-\mathrm{N}^{\circ} 1$ 
induites à supports compacts de $\Lambda$-représentations finies de sous-groupes ouverts compacts $K_{j}$. Ces dernières admettent elle-mêmes des résolutions par des $\Lambda K_{j}$-modules lisses projectifs de type fini, si bien qu'on obtient au final des résolutions (en général infinie) des $H_{c}^{q}$ par des induites compactes de représentations projectives de type fini. Comme par ailleurs les $H_{c}^{q}$ sont $\Lambda$-admissibles, on en déduit que chaque $\operatorname{Ext}_{\Lambda P G_{d}}^{q-k}\left(H_{c}^{q}, H_{c}^{k}\right)$ est de type fini sur $\Lambda$.

Ainsi dans le cas $\Lambda=\mathbb{Z}_{l}$, le groupe $\mathcal{U}$ est pro-l, et comme tout morphisme entre groupes profinis est automatiquement continu, $\gamma_{\mid I_{K}}$ est continu et par conséquent se factorise par $\mathbb{Z}_{l}(1)$. Comme $R \Gamma_{c}\left(\Omega_{K}^{d-1, \mathrm{ca}}, \Lambda\right) \simeq R \Gamma_{c}\left(\Omega_{K}^{d-1, \mathrm{ca}}, \mathbb{Z}_{l}\right) \otimes_{\mathbb{Z}_{l}}^{L} \Lambda$, on en déduit la même factorisation pour tout $\Lambda$.

Dans le cas où $d$ ! est inversible, le morphisme $N:=\log (\gamma(\mu)) \otimes \mu^{-1} \in \mathcal{N}(-1)$ est indépendant du choix d'un générateur topologique $\mu$ de $\mathbb{Z}_{l}(1)$, et par densité de $\mathbb{Z}$ dans $\mathbb{Z}_{l}$, l'action de $I_{K}$ est bien donnée par la formule de l'énoncé.

Enfin, pour tout $w \in W_{K}$, l'égalité $\gamma(w) \log (\gamma(\mu)) \gamma\left(w^{-1}\right)=|w| \log (\gamma(u))$ s'écrit encore $\gamma(w)(-1) \circ N=N \circ \gamma(w)$, d'où la $W_{K}$-équivariance de $N$.

Pour expliciter l'action de $W_{K}$, et en particulier le morphisme $N$, sur le complexe de cohomologie, nous supposerons dorénavant $\Lambda$ fortement banal pour $G L_{d}(K)$. La première étape consiste à remarquer que le complexe $R \Gamma_{c}\left(\Omega_{K}^{d-1, \text { ca }}, \Lambda\right)$ est scindable.

4.2.2. Proposition. - Il existe un isomorphisme dans $D^{b}\left(\Lambda P G_{d}\right)$

$$
R \Gamma_{c}\left(\Omega_{K}^{d-1, \mathrm{ca}}, \Lambda\right) \stackrel{\sim}{\longrightarrow} \bigoplus_{i=0}^{d-1} \pi_{\{1, \ldots, i\}}^{\Lambda}(-i)[-d+1-i]
$$

qui induit en cohomologie les isomorphismes de Schneider-Stuhler 3.1.1.

Empressons-nous de préciser qu'un tel isomorphisme est loin d'être unique, mais nous en exhiberons certains meilleurs que les autres. Nous allons donner deux preuves indépendantes de ce fait. La première repose sur un critère général de scindage et sur un calcul explicite des groupes d'extensions entre les diverses représentations $\pi_{I}, I \subseteq S$ : on obtient un argument purement algébrique qui montre que tout complexe de représentations lisses de $P G_{d}$ ayant pour cohomologie les $H_{c}^{i}\left(\Omega_{K}^{d-1, \text { ca }}, \Lambda\right)$ est scindable. La deuxième est d'origine plus géométrique car on utilise l'action d'un relèvement de Frobenius sur $R \Gamma_{c}\left(\Omega_{K}^{d-1, c a}, \Lambda\right)$; elle donne plus d'informations qui seront utiles par la suite.

Preuve algébrique : Nous utiliserons le critère suivant permettant de scinder un complexe cohomologiquement borné $\mathcal{C}^{\bullet}$ d'une catégorie dérivée assez générale. La preuve est facile et est donnée en A.1 : c'est le corollaire A.1.3 spécialisé dans notre situation.

Si pour tout couple $(i, j) \in \mathbb{Z}^{2}$, on a $\operatorname{Ext}^{i-j-1}\left(\mathcal{H}^{i}\left(\mathcal{C}^{\bullet}\right), \mathcal{H}^{j}\left(\mathcal{C}^{\bullet}\right)\right)=0$, alors $\mathcal{C}^{\bullet}$ est scindable.

D'après le théorème 2.1.4 et la description 3.1.1 de la cohomologie de $\Omega_{K}^{d-1}$, on a pour $i, j \in\{0, \ldots, d-1\}$

$$
\operatorname{Ext}_{P G_{d}}^{*}\left(H_{c}^{d-1+i}\left(\Omega_{K}^{d-1, \mathrm{ca}}, \Lambda\right), H_{c}^{d-1+j}\left(\Omega_{K}^{d-1, \mathrm{ca}}, \Lambda\right)\right)= \begin{cases}\Lambda & \mathrm{si} *=|i-j| \\ 0 & \mathrm{si} * \neq|i-j|\end{cases}
$$

ce qui implique en particulier que le critère de scindage ci-dessus est vérifié.

Preuve géométrique : Soit $\phi$ un relèvement de Frobenius géométrique. La description de Schneider-Stuhler montre que pour $i=0, \ldots, d-1$, l'endomorphisme $\mathcal{H}^{d-1+i}(\gamma(\phi))$ induit par $\gamma(\phi)$ sur $H_{c}^{d-1+i}\left(\Omega_{K}^{d-1, \mathrm{ca}}, \Lambda\right)$ est la multiplication par $q^{i}$. Comme $\Lambda$ est supposé banal, l'entier $q^{j}-q^{i}$ est inversible dans $\Lambda$ pour tous $0 \leqslant i<j \leqslant d-1$ et on a donc l'égalité $\left(X-q^{i}\right) \Lambda[X]+\left(X-q^{j}\right) \Lambda[X]=\Lambda[X]$. On peut alors encore utiliser un résultat très général sur les complexes donné par le lemme A.1.4. Ce résultat assure que 
(i) $\gamma(\phi)$ est annulé par le polynôme $\prod_{i=0}^{d-1}\left(X-q^{i}\right)$ et par conséquent est un endomorphisme semi-simple de $R \Gamma_{c}\left(\Omega_{K}^{d-1, \text { ca }}, \Lambda\right)$.

(ii) Il existe un unique isomorphisme comme dans la proposition

$$
\alpha_{\phi}: R \Gamma_{c}\left(\Omega_{K}^{d-1, \mathrm{ca}}, \Lambda\right) \stackrel{\sim}{\longrightarrow} \bigoplus_{i=0}^{d-1} \pi_{\{1, \ldots, i\}}^{\Lambda}(-i)[-d+1-i]
$$

tel que de plus, l'endomorphisme $\alpha_{\phi}^{-1} \gamma(\phi) \alpha_{\phi}$ de l'objet $\bigoplus_{i=0}^{d-1} \pi_{\{1, \ldots, i\}}^{\Lambda}(-i)[-d+1-i]$ soit donné par la multiplication par $q^{i}$ sur chaque $\pi_{\{1, \ldots, i\}}^{\Lambda}(-i)[-d+1-i]$.

Un isomorphisme $\alpha$ comme dans la proposition ci-dessus sera appelé un scindage ${ }^{1}$ de $R \Gamma_{c}\left(\Omega_{K}^{d-1, \mathrm{ca}}, \Lambda\right)$. Tout scindage induit en particulier un isomorphisme

$$
\alpha_{*}: \operatorname{End}_{D^{b}\left(P G_{d}\right)}\left(R \Gamma_{c}\left(\Omega_{K}^{d-1, \mathrm{ca}}, \Lambda\right)\right) \stackrel{\sim}{\longrightarrow} \operatorname{End}_{D^{b}\left(P G_{d}\right)}\left(\bigoplus_{i=0}^{d-1} \pi_{\{1, \ldots, i\}}^{\Lambda}(-i)[-i]\right) .
$$

Il se trouve qu'on peut décrire très explicitement le membre de droite. On peut tout d'abord le réécrire

$$
\operatorname{End}_{D^{b}\left(P G_{d}\right)}\left(\bigoplus_{i=0}^{d-1} \pi_{\{1, \ldots, i\}}^{\Lambda}(-i)[-i]\right)=\bigoplus_{0 \leqslant i \leqslant j \leqslant d-1} \operatorname{Ext}_{P G_{d}}^{j-i}\left(\pi_{\{1, \ldots, j\}}^{\Lambda}, \pi_{\{1, \ldots, i\}}^{\Lambda}\right)(j-i)
$$

le produit sur le terme de droite étant le $\cup$-produit. On peut donc utiliser le théorème 2.1.4. Choisissons pour $i=0, \ldots, d-1$ des générateurs

$$
\beta_{i, i+1} \in \operatorname{Ext}_{P G_{d}}^{1}\left(\pi_{\{1, \ldots, i+1\}}^{\Lambda}, \pi_{\{1, \ldots, i\}}^{\Lambda}\right)
$$

et posons pour tous $0 \leqslant i<j \leqslant d-1$

$$
\beta_{i, j}:=\beta_{i, i+1} \cup \cdots \cup \beta_{j-1, j} \in \operatorname{Ext}_{P G_{d}}^{j-i}\left(\pi_{\{1, \ldots, j\}}^{\Lambda}, \pi_{\{1, \ldots, i\}}^{\Lambda}\right) .
$$

C'est donc un générateur du $\Lambda$-module de droite, d'après 2.1.4. Enfin pour $i=0, \ldots, d-1$, soit $\beta_{i i}$ l'élément unité de l'anneau $\operatorname{Ext}_{P G_{d}}^{0}\left(\pi_{\{1, \ldots, i\}}^{\Lambda}, \pi_{\{1, \ldots, i\}}^{\Lambda}\right)$. Notons maintenant $\mathcal{T}_{d}(\Lambda)$ la $\Lambda$-algèbre des matrices $d \times d$ triangulaires supérieures, et $\left(E_{i j}\right)_{0 \leqslant i \leqslant j \leqslant d-1}$ sa base « canonique ». Nous avons tout fait pour que l'application $\Lambda$-linéaire

$$
\begin{aligned}
\beta: \mathcal{T}_{d}(\Lambda) & \rightarrow \bigoplus_{0 \leqslant i \leqslant j \leqslant d-1} \operatorname{Ext}_{P G_{d}}^{j-i}\left(\pi_{\{1, \ldots, j\}}^{\Lambda}, \pi_{\{1, \ldots, i\}}^{\Lambda}\right), \\
E_{i j} & \mapsto \beta_{i j}
\end{aligned}
$$

soit un isomorphisme de $\Lambda$-algèbres. Celui-ci dépend du choix de générateurs (4.2.4); un autre choix reviendrait à composer $\beta$ avec la conjugaison par une matrice diagonale inversible. Introduisons maintenant l'algèbre $\widetilde{\mathcal{T}}_{d}(\Lambda)=\bigoplus_{i \leqslant j} \Lambda(j-i)$ des matrices triangulaires supérieures «tordues ». Par la discussion précédente, tout scindage $\alpha$ de $R \Gamma_{c}\left(\Omega_{K}^{d-1, \text { ca }}, \Lambda\right)$ et tout choix de générateurs (4.2.4) induisent donc un isomorphisme

$$
\tilde{\beta}^{-1} \alpha_{*}: \operatorname{End}_{D^{b}\left(P G_{d}\right)}\left(R \Gamma_{c}\left(\Omega_{K}^{d-1, \text { ca }}, \Lambda\right)\right) \stackrel{\sim}{\longrightarrow} \widetilde{\mathcal{T}}_{d}(\Lambda) .
$$

\footnotetext{
${ }^{1}$ La terminologie adoptée ici coïncide bien avec celle de la section A.1, malgré la formulation légèrement différente.

$4^{\mathrm{e}}$ SÉRIE - TOME $39-2006-\mathrm{N}^{\circ} 1$
} 
On veut maintenant expliciter l'action de $W_{K}$ sur $R \Gamma_{c}\left(\Omega_{K}^{d-1, \mathrm{ca}}, \Lambda\right)$ au moyen d'un tel isomorphisme. Remarquons que les contragrédientes des représentations $\tau_{I}^{\Lambda, \phi}: W_{K} \rightarrow \operatorname{End}_{\Lambda}(V)$ que nous avons explicitement définies en 4.1.1 se factorisent par des morphismes $\check{\tau}_{I}^{\Lambda, \phi}: W_{K} \rightarrow$ $\widetilde{\mathcal{T}}_{d}(\Lambda)$.

4.2.6. Proposition. - Pour tout relèvement de Frobenius géométrique $\phi$, il existe un unique scindage $\alpha_{\phi}$ de $R \Gamma_{c}\left(\Omega_{K}^{d-1, \mathrm{ca}}, \Lambda\right)$ et un unique choix de générateurs (4.2.4) définissant un isomorphisme $\beta_{\phi}$ comme en (4.2.5) tels que le diagramme suivant soit commutatif

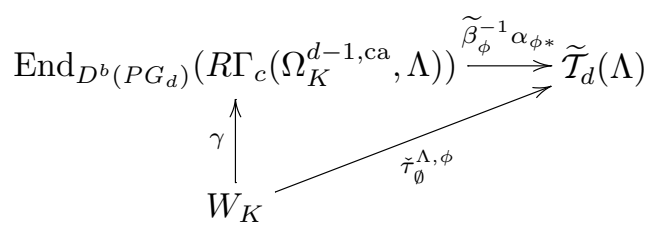

De plus, le choix de générateurs (et donc $\beta_{\phi}$ ) est en fait indépendant de $\phi$.

Rappelons ici que $\tau_{\emptyset}^{\Lambda, \phi}$ correspond à la représentation «spéciale» (indécomposable) de dimension $d$ de $W_{K}$. En particulier cette proposition montre que l'inertie n'agit pas trivialement sur $R \Gamma_{c}\left(\Omega_{K}^{d-1, \mathrm{ca}}, \Lambda\right)$ bien qu'elle agisse trivialement sur les groupes de cohomologie.

Début de la preuve : Commençons par prouver les propriétés d'unicité. En ce qui concerne le scindage, nous n'avons pas d'autre choix que de prendre celui de (4.2.3) puisque c'est l'unique scindage qui «diagonalise » $\phi$, au sens où pour tout choix de $\beta$, la matrice $\tilde{\beta}^{-1} \alpha_{\phi *}(\phi)$ est diagonale d'éléments diagonaux $\tilde{\beta}^{-1} \alpha_{\phi *}(\phi)_{i i}=q^{i}$ pour $i=0, \ldots, d-1$. Supposons maintenant qu'on ait trouvé un $\beta_{\phi}$, associé à un certain choix de générateurs, rendant commutatif le diagramme de l'énoncé. Changer ce choix revient à conjuguer $\beta_{\phi}$ par une matrice diagonale non centrale. Or, $\tilde{\beta}_{\phi}^{-1} \alpha_{\phi *}(N)$ est un nilpotent régulier donc son centralisateur dans le groupe des matrices diagonales est justement le sous-groupe des matrices centrales. On en déduit l'unicité de $\beta_{\phi}$. Montrons maintenant que $\beta_{\phi}$ doit être indépendant de $\phi$. Soit $i \in I_{K}$; rappelons que par $[16,8.4 .2]$ on a

$$
\forall w \in W_{K}, \quad \tau_{\emptyset}^{\Lambda, i \phi}(w)=\tau_{\emptyset}^{\Lambda, \phi}(i)^{\frac{1}{1-q}} \tau_{\emptyset}^{\Lambda, \phi}(w) \tau_{\emptyset}^{\Lambda, \phi}(i)^{\frac{1}{q-1}} .
$$

De même, en utilisant la propriété $\gamma(\phi) \gamma(i) \gamma(\phi)^{-1}=\gamma(i)^{q}$, on vérifie que

$$
\alpha_{i \phi}=\alpha_{\phi} \circ \operatorname{ad}\left(\gamma(i)^{\frac{1}{1-q}}\right)
$$

(On utilise le fait que $d ! \in \Lambda^{\times}$et que $\gamma(i)$ est unipotent d'ordre $\leqslant d$ pour définir ses puissances rationnelles.) À partir de ces deux équations, un calcul montre que $\beta_{i \phi}$ fait aussi commuter le diagramme de l'énoncé et par unicité on en conclut que $\beta_{\phi}=\beta_{i \phi}$.

Passons maintenant à l'existence de $\beta$, et pour cela, commençons avec $\beta$ quelconque. Remarquons que par définition de $\alpha_{\phi}$, l'isomorphisme

$$
\tilde{\beta}^{-1} \alpha_{\phi *}: \operatorname{End}_{D^{b}\left(\Lambda P G_{d}\right)}\left(R \Gamma_{c}\right) \stackrel{\sim}{\longrightarrow} \bigoplus_{0 \leqslant i \leqslant j \leqslant d-1} \Lambda(j-i)
$$

transporte l'action de $\phi$ par conjugaison sur son action «naturelle» sur le membre de droite. On veut alors expliciter le morphisme $N$ du lemme 4.2.1. Avec les notations de la preuve de ce 
lemme, l'isomorphisme $\tilde{\beta}^{-1} \alpha_{\phi *}$ ci-dessus induit un isomorphisme

$$
\mathcal{N}(-1) \stackrel{\sim}{\longrightarrow} \bigoplus_{0 \leqslant i<j \leqslant d-1} \Lambda(j-i-1) .
$$

Le morphisme $N$ est $W_{K}$-équivariant donc son image $\tilde{\beta} \alpha_{\phi *}(N)$ appartient au sous- $\Lambda$-module $\bigoplus_{j-i=1} \Lambda$ du membre de droite ; c'est une matrice «sur-diagonale » de la forme $\tilde{\beta}^{-1} \alpha_{\phi *}(N)=$ $\sum_{i=1}^{d-1} a_{i} E_{i-1, i}$. Une telle matrice est conjuguée par une matrice diagonale à l'élément régulier $N_{\emptyset}=\sum_{i=1}^{d-1} E_{i, i-1}$ si et seulement si le produit $\prod_{i=1}^{d-1} a_{i}$ est inversible dans $\Lambda$, ou encore si et seulement si l'image de $\tilde{\beta}^{-1} \alpha_{\phi *}(N)^{d-1}$ dans $\mathcal{T}_{d}(\Lambda / \mathfrak{m})$ est non nulle, en notant $\mathfrak{m}$ l'idéal maximal de $\Lambda$.

En résumé la proposition 4.2.6 est équivalente à la suivante (avec les notations de la preuve du lemme 4.2.1) :

4.2.7. Proposition. - Si $\mathfrak{m}$ désigne l'idéal maximal de $\Lambda, N^{d-1} \notin \mathfrak{m} \mathcal{N}(-1)$.

Évidemment dans le cas où $\Lambda$ est un corps, cela dit simplement que $N^{d-1}$ est non-nul. La preuve de cette proposition occupe la section suivante.

\subsection{Utilisation de la suite spectrale de Rapoport-Zink}

La stratégie pour prouver 4.2.7 repose sur la suite spectrale de Rapoport-Zink [35] associée aux schémas formels semi-stables du type $\Omega_{K}^{d-1} / \Gamma$ où $\Gamma \subset P G L_{d}(K)$ est un sous-groupe discret sans torsion. En fait, si l'on s'intéresse seulement au cas où $\Lambda$ est un corps $l$-adique, on pourrait s'en sortir en prenant $\Gamma$ cocompact, de sorte que, le quotient étant alors algébrisable, on pourrait utiliser verbatim [35]. Mais pour traiter le cas entier ou de torsion, il faut contrôler certains dénominateurs et cela s'avère plus facile si l'on quotiente par un sous-groupe discret plus simple; nous utiliserons un sous-groupe discret cocompact et sans torsion d'un tore maximal de $P G_{d}$. Le schéma formel obtenu $\widehat{\Omega} / \Gamma$ n'est plus algébrisable, mais nous allons vérifier que le formalisme de Rapoport-Zink s'y applique sans problèmes. Les définitions et résultats qui suivent ne sont certainement pas optimaux, mais suffiront pour nos affaires.

4.3.1. Définition. - Un schéma formel $\mathfrak{X}$ quasi-séparé au-dessus de $\operatorname{Spf}\left(\mathcal{O}_{K}\right)$ sera dit fortement semi-stable si :

(i) localement pour la topologie de Zariski, $\mathfrak{X}$ est étale au-dessus d'un $\operatorname{Spf}\left(\mathcal{O}_{K}\left\langle T_{1}, \ldots, T_{n}\right\rangle /\right.$ $\left.\left(T_{1} \times \cdots \times T_{m}-\varpi\right)\right)$.

(ii) Les composantes irréductibles de la fibre spéciale $Y=\mathfrak{X} \times_{\mathcal{O}_{K}} k$ sont propres et lisses de dimension constante sur $k$.

Soit $I$ l'ensemble des composantes irréductibles de $Y$. On met une structure simpliciale sur $I$ en déclarant qu'un sous-ensemble $J \subset I$ est un simplexe si $Y_{J}:=\bigcap_{j \in J} Y_{j} \neq \emptyset$. Dans ce cas, si $d-1$ désigne la dimension commune des composantes irréductibles de $Y$, alors $Y_{J}$ est une sous-

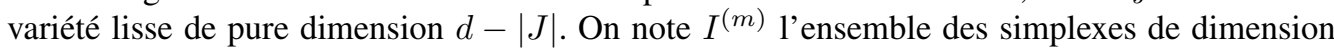
$m-1$ (donc de cardinal $m$ ) de $I$.

Notons maintenant $X$ la fibre générique au sens de Berkovich de $\mathfrak{X}$ : c'est un espace $K$-analytique lisse.

4.3.2. Proposition. - Soit $\mathfrak{X}$ un schéma formel fortement semi-stable sur $\mathcal{O}_{K}$. Les $W_{K}$ modules $H_{c}^{q}\left(X^{\mathrm{ca}}, \Lambda\right)$ sont modérément ramifiés et il existe une suite spectrale

$$
E_{1}^{-r, q+r}=\bigoplus_{k \geqslant \sup (0,-r)} \bigoplus_{J \in I^{(r+2 k+1)}} H^{q+1-|J|}\left(Y_{J}^{\mathrm{ca}}, \Lambda(-r-k)\right) \Longrightarrow H_{c}^{q}\left(X^{\mathrm{ca}}, \Lambda\right)
$$

$4^{\text {e }}$ SÉRIE - TOME $39-2006-\mathrm{N}^{\circ} 1$ 
dont la différentielle $d_{1}$ de degré $(-1,1)$ en $(r, q)$ s'écrit

$$
\begin{aligned}
d_{1}^{-r, q+r}= & \sum_{k \geqslant \sup (0,-r)}(-1)^{k} \sum_{J \in I^{(r+2 k+1)}}\left(\sum_{K \supset J} \varepsilon_{J K} \operatorname{Res}_{J K}\right) \\
& +\sum_{k \geqslant \sup (0,-r+1)}(-1)^{r+k} \sum_{J \in I^{(r+2 k+1)}}\left(\sum_{K \subset J} \varepsilon_{J K} \mathrm{Gys}_{J K}\right)
\end{aligned}
$$

où

$$
\operatorname{Res}_{J K}: H^{q+1-|J|}\left(Y_{J}^{\mathrm{ca}}, \Lambda\right) \rightarrow H^{q+1-|J|}\left(Y_{K}^{\mathrm{ca}}, \Lambda\right)=H^{(q+1)+1-|K|}\left(Y_{K}^{\mathrm{ca}}, \Lambda\right)
$$

est le morphisme de restriction associé à $Y_{K} \hookrightarrow Y_{J}$ lorsque $J \subset K$,

$$
\operatorname{Gys}_{J K}: H^{q+1-|J|}\left(Y_{J}^{\mathrm{ca}}, \Lambda\right) \rightarrow H^{q+1-|J|+2}\left(Y_{K}^{\mathrm{ca}}, \Lambda(1)\right)=H^{(q+1)+1-|K|}\left(Y_{K}^{\mathrm{ca}}, \Lambda(1)\right)
$$

est le morphisme de Gysin associé à $Y_{J} \hookrightarrow Y_{K}$ lorsque $K \subset J$, et les $\varepsilon_{J, K}$ sont des signes donnés par le choix d'une orientation de l'ensemble simplicial $I^{(\bullet)}$.

De plus, si $\mu$ est un générateur topologique de $\mathbb{Z}_{l}(1)$, l'action de $\mu-1$ sur l'aboutissement est induite par l'endomorphisme $\nu$ de degré $(-2,0)$ en $(r, q)$ défini par

$$
\nu^{-r, q+r}=\sum_{k \geqslant \sup (0,-r+1)} \sum_{J \in I^{(r+2 k+1)}} \operatorname{Id}_{\left.H^{q+1-|J|}\left(Y_{J}^{\mathrm{ca}}, \Lambda(-r-k)\right)\right)} \otimes \mu .
$$

Preuve. - Supposons tout d'abord $\Lambda$ fini. Par définition, un schéma formel $\mathfrak{X}$ fortement semistable est localement de présentation finie. On peut donc lui appliquer le formalisme des cycles évanescents de Berkovich [3]. Celui-ci fournit en particulier deux foncteurs

- cycles évanescents absolus : $R \Psi_{\eta}: D^{+}(X, \Lambda) \rightarrow D^{+}\left(Y^{\mathrm{ca}}, \Lambda W_{K}\right)$ où le terme de droite désigne la catégorie dérivée des $\Lambda$-faisceaux étales $W_{K}$-équivariants sur $Y^{\text {ca }}$

- cycles évanescents non-ramifiés $R \Psi: D^{+}(X, \Lambda) \rightarrow D^{+}\left(Y^{\text {ca }}, \Lambda W_{k}\right)$ où $W_{k}$ est le groupe de Weil de $k$ (libre engendré par le Frobenius). Ceux-ci seraient notés $\bar{i}_{k^{s}}^{*} R \Theta$ dans la notation de Berkovich.

Ces foncteurs satisfont la relation $R \Psi(-) \stackrel{\sim}{\longrightarrow} R \operatorname{Hom}_{I_{K}}\left(\Lambda, R \Psi_{\eta}(-)\right)$, voir [3, 4.8]. D'après notre hypothèse, les composantes irréductibles de la fibre spéciale $Y$ sont propres. D'après Berkovich [7, 2.5], on a donc un isomorphisme

$$
R \Gamma_{c}\left(Y^{\mathrm{ca}}, R \Psi_{\eta}(\Lambda)\right) \stackrel{\sim}{\longrightarrow} R \Gamma_{c}\left(X^{\mathrm{ca}}, \Lambda\right) .
$$

Notons que la cohomologie «à supports compacts» de $Y^{\text {ca }}$ qui apparait dans le membre de gauche est définie de manière naïve comme le foncteur dérivé des sections à support propres, car $Y^{\text {ca }}$ est réunion croissante de fermés de type fini, donc propres; il n'est donc pas question de «compactifier» $Y^{\mathrm{ca}}$. Soit $\mathfrak{U}$ un sous-schéma formel ouvert de $\mathfrak{X}$ muni d'un morphisme étale $\mathfrak{U} \rightarrow \operatorname{Spf}\left(\mathcal{O}_{K}\left\langle T_{1}, \ldots, T_{n}\right\rangle /\left(T_{1} \times \cdots \times T_{m}-\varpi\right)\right)$. Le théorème de comparaison de Berkovich [3, 5.1] et le théorème de pureté de Rapoport-Zink [35, 2.21,2.25] montrent que $I_{K}$ agit trivialement sur les faisceaux de cohomologie $R^{q} \Psi_{\eta}(\Lambda)_{\mid\left(Y^{\text {ca }} \cap \mathfrak{U}\right)}$. Comme $\mathfrak{X}$ est supposé recouvert par de tels ouverts, l'action de $I_{K}$ sur les $R^{q} \Psi_{\eta}(\Lambda)$ est elle aussi triviale.

À partir de là et de (4.3.3), on peut appliquer le formalisme développé par Rapoport-Zink dans la section 1 de [35]. En suivant la section 2 de loc. cit., on remarque que l'existence de la suite spectrale annoncée est une conséquence formelle de l'existence d'isomorphismes

$$
R^{q} \Psi(\Lambda) \stackrel{\sim}{\longrightarrow} a_{q, *} \Lambda(-q) \quad \text { où } a_{q}: \bigsqcup_{J \in I^{(q)}} Y_{J} \rightarrow Y
$$


rendant commutatifs les diagrammes

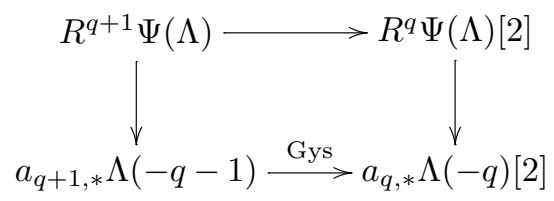

et

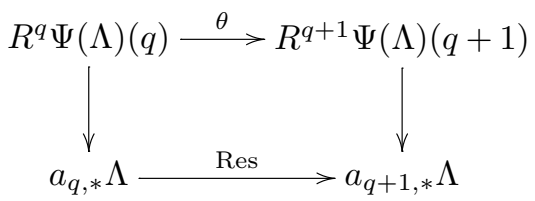

Dans le premier diagramme, la flèche du haut est l'extension canonique donnée par le complexe $\tau_{\leqslant q} \tau_{>q} R \Psi$ et celle du bas est une somme alternée de morphismes de Gysin (obtenus grâce à la pureté, cf. [35, p. 36]). Dans le second diagramme le morphisme $\theta$ est le cup-produit par l'extension canonique $\Lambda \rightarrow \Lambda(1)[1]$ cf. [35, p. 26] et la flèche du bas est une somme alternée de morphismes de restrictions. Les deux sommes alternées dépendent de l'orientation choisie sur l'ensemble simplicial $I^{(\bullet)}$ et les isomorphismes (4.3.4) doivent donc en dépendre aussi. Fixons dorénavant une telle orientation.

D'après la section 2 de Rapoport-Zink [35] et le théorème de comparaison de Berkovich, on a pour toute carte $\mathfrak{U}$ comme ci-dessus, des isomorphismes

$$
R^{q} \Psi(\Lambda)_{\mid \mathfrak{U} \cap Y^{\mathrm{ca}}} \stackrel{\sim}{\longrightarrow} a_{q, *} \Lambda(-q)_{\mid \mathfrak{U} \cap Y^{\mathrm{ca}}}
$$

rendant commutatifs les diagrammes correspondants. Comme les deux diagrammes en question sont de nature locale, il suffira, pour conclure, de recoller les isomorphismes (4.3.5). De plus, ces isomorphismes pour $q>1$ sont déduits du cas $q=1$ par cup-produit, cf. [28]. Il nous suffit donc de recoller les isomorphismes

$$
R^{1} \Psi(\Lambda)_{\mid \mathfrak{U} \cap Y^{\text {ca }}} \stackrel{\sim}{\sim} a_{1, *} \Lambda(-1)_{\mid \mathfrak{U} \cap Y^{\text {ca }}} .
$$

En fait, nous allons suivre T. Saito dans [37, Prop. 1.1.2] pour construire un morphisme $a_{1, *} \Lambda \rightarrow R \Psi(\Lambda)(1)$ qui induit sur chaque ouvert $\mathfrak{U}$ l'inverse de l'isomorphisme ci-dessus.

Pour cela, nous supposerons $\Lambda=\mathbb{Z} / l^{n} \mathbb{Z}$, le cas général ( $\Lambda$ fini) s'en déduisant par fonctorialité. Soit $Y_{i}$ une composante irréductible de $Y$. Elle est donnée par un idéal localement principal $\mathcal{I}_{i}$ du faisceau d'anneaux adiques $\mathcal{O}_{\mathfrak{X}}$. En particulier, sur une carte formelle $\mathfrak{U}=\operatorname{Spf}\left(\mathcal{O}_{\mathfrak{U}}\right)$ de $\mathfrak{X}$ comme ci-dessus, l'idéal $\mathcal{I}_{i} \mathcal{O}_{\mathfrak{U}}$ est engendré par un élément $f_{i, \mathfrak{U}} \in \mathcal{O}_{\mathfrak{U}}$. Cet élément est inversible dans l'algèbre affinoïde $\mathcal{O}_{U}:=\mathcal{O}_{\mathfrak{U}} \otimes_{\mathcal{O}} K$ de l'espace rigide $U$ fibre générique de $\mathfrak{U}$. La suite exacte de Kummer fournit un morphisme bord

$$
\mathcal{O}_{U}^{\times} \stackrel{\partial}{\rightarrow} H^{1}(U, \Lambda(1))
$$

que l'on peut composer avec l'application canonique (donnée par définition même des cycles évanescents de Berkovich)

$$
H^{1}(U, \Lambda) \rightarrow \Gamma\left(\mathfrak{U} \cap Y^{\mathrm{ca}}, R^{1} \Psi(\Lambda)\right) .
$$

On obtient ainsi un élément $\partial f_{i, \mathfrak{U}} \in \Gamma\left(\mathfrak{U} \cap Y^{\mathrm{ca}}, R^{1} \Psi(\Lambda)\right)(1)$. Si $\mathfrak{W}$ est un ouvert étale de $\mathfrak{U}$ disjoint de $Y_{i}$ et suffisamment petit pour que $H^{1}(\mathfrak{W}, \Lambda)=0$, alors l'élément $\left(\partial f_{i, \mathfrak{U}}\right)_{\mid \mathfrak{W}}$ est nul car $f_{i, \mathfrak{U}}$ est inversible dans $\mathcal{O}_{\mathfrak{W}}$. Ainsi, $\partial f_{i, \mathfrak{U}}$ est à support dans $Y_{i}$, i.e. appartient au module $\Gamma_{Y_{i}^{\text {ca }}}\left(\mathfrak{U} \cap Y^{\mathrm{ca}}, R \Psi^{1}(\Lambda)\right)(1)$. On en déduit par adjonction un morphisme de faisceaux $i_{Y_{i}, *}(\Lambda)_{\mid \mathfrak{U}} \rightarrow R^{1} \Psi(\Lambda)(1)_{\mid \mathfrak{U}}$. Or, le générateur $f_{i, \mathfrak{U}}$ de $\mathcal{I}_{i} \mathcal{O}_{\mathfrak{U}}$ est unique à multiplication par un inversible de $\mathcal{O}_{\mathfrak{U}}$ près. En recouvrant $\mathfrak{U}$ par des ouverts étales $\mathfrak{W}$ tels que $H^{1}(\mathfrak{W}, \Lambda)=0$, on en

$4^{\mathrm{e}}$ SÉRIE - TOME $39-2006-\mathrm{N}^{\circ} 1$ 
déduit que $\partial f_{i, \mathfrak{U}}$ est en fait indépendant du choix de l'équation locale $f_{i, \mathfrak{U}}$ de $Y_{i}$. On peut donc recoller les morphismes ainsi obtenus pour toutes les cartes formelles $\mathfrak{U}$ comme ci-dessus pour en déduire un morphisme global $i_{Y_{i}, *} \Lambda \rightarrow R \Psi^{1}(\Lambda)(1)$.

Par construction et par [37, 1.1.2], la somme $a_{1, *}(\Lambda) \rightarrow R \Psi(\Lambda)(1)$ de ces morphismes induit sur chaque carte $\mathfrak{U}$ l'isomorphisme inverse de (4.3.5) pour $q=1$.

Expliquons maintenant comment passer au cas où $\Lambda$ est plat sur $\mathbb{Z}_{l}$. Il suffit bien sûr de traiter le cas $\mathbb{Z}_{l}$. Remarquons que lorsque $\mathfrak{X}$ est quasi compact (et donc $I$ est fini), on a $H_{c}^{q}\left(X^{\mathrm{ca}}, \mathbb{Z}_{l}\right) \stackrel{\sim}{\longrightarrow} \varliminf_{\longrightarrow} H_{c}^{q}\left(X^{\mathrm{ca}}, \mathbb{Z} / l^{n} \mathbb{Z}\right)$ et les systèmes projectifs $H^{q}\left(Y^{\mathrm{ca}}, \mathbb{Z} / l^{n} \mathbb{Z}\right)$ sont AR-l-adiques; on en déduit par passage à la limite comme dans le cas algébrique la suite spectrale voulue pour $\Lambda=\mathbb{Z}_{l}$. Dans le cas général, on choisit une suite croissante $\left(I_{n}\right)_{n \in \mathbb{N}}$ de sous-ensembles finis de $I$ dont la réunion est $I$. Notons $Y_{n}$ les fermés de la fibre spéciale et $X_{n}$ les ouverts de $X$ correspondants. D'après [7, Cor. 2.5], il y a des isomorphismes canoniques $R \Gamma_{Y_{n}}\left(Y^{\text {ca }}, R \Psi_{\eta}\left(\mathbb{Z} / l^{n} \mathbb{Z}\right)\right) \stackrel{\sim}{\longrightarrow} R \Gamma_{\overline{X_{n}}}\left(X^{\text {ca }}, \mathbb{Z} / l^{m} \mathbb{Z}\right)$ dont la «limite inductive» est l'isomorphisme (4.3.3). D'après la description des cycles évanescents, on a donc pour tous $n$ et $m$ une suite spectrale comme dans l'énoncé sauf que l'on y remplace $H^{*}\left(Y_{J}^{\mathrm{ca}}, \Lambda\right)$ par $H_{Y_{n}}^{*}\left(Y_{J}^{\mathrm{ca}}, \mathbb{Z} / l^{m} \mathbb{Z}\right)$ dans le terme $E_{1}$ et $H^{*}\left(X^{\mathrm{ca}}, \Lambda\right)$ par $H_{X_{n}}^{*}\left(X^{\mathrm{ca}}, \mathbb{Z} / l^{m} \mathbb{Z}\right)$ dans l'aboutissement. Puisque l'ensemble des $J \subset I$ tels que $Y_{J} \cap Y_{n} \neq \emptyset$ est fini, on obtient en passant à la limite projective sur $m$ une suite spectrale similaire avec $\mathbb{Z}_{l}$ à la place de $\mathbb{Z} / l^{m} \mathbb{Z}$. On a alors un système de suites spectrales indexé par $n$ dont la limite inductive est la suite spectrale voulue.

\subsubsection{Preuve de la proposition 4.2 .7}

Dans les cas particuliers $(r, q)=(d-1, d-1)$ et $(r, q)=(1-d, d-1)$, la description de la différentielle et de l'opérateur $\nu$ de la suite spectrale 4.3.2 s'insère dans le diagramme suivant :

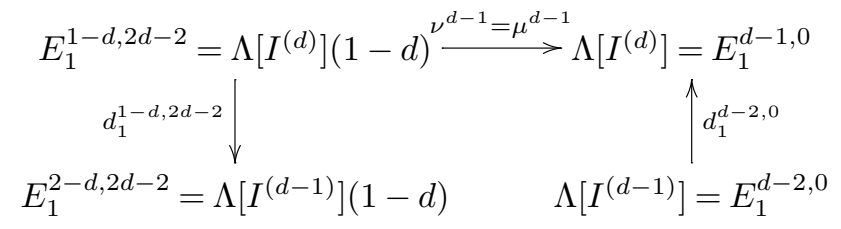

où $\Lambda[?]$ désigne le $\Lambda$-module des fonctions à support fini sur l'ensemble ? et

$$
d_{1}^{1-d, 2 d-2}(f)(K)=\sum_{J \supset K} \varepsilon_{J K} \cdot f(J) \quad \text { et } \quad d_{1}^{d-2,0}(g)(J)=\sum_{K \subset J} \varepsilon_{J K} \cdot g(K) .
$$

Munissons alors $\Lambda[?]$ de la forme bilinéaire «canonique» pour laquelle la base ? est autoduale, et posons ' $E_{1}^{1-d, d-2}:=E_{1}^{1-d, 2 d-2}(d-1)$ ainsi que ' $d_{1}^{1-d, 2 d-2}:=d_{1}^{1-d, 2 d-2}(d-1)$. On constate que ' $d_{1}^{1-d, 2 d-2}$ et $d_{1}^{d-2,0}$ sont adjointes l'une de l'autre. En particulier, on a $\operatorname{im}\left(d_{1}^{d-2,0}\right) \subset$ $\operatorname{ker}\left({ }^{\prime} d_{1}^{1-d, 2 d-2}\right)^{\perp}$. Puisque $E_{2}^{1-d, 2 d-2}=\operatorname{ker} d_{1}^{1-d, 2 d-2}$ et $E_{2}^{d-1,0}=\operatorname{coker} d_{1}^{d-2,0}$, on en déduit le lemme suivant

4.3.7. Lemme (Rappelons que $\mathfrak{m}$ est l'idéal maximal de $\Lambda$ ). -

$$
\text { Si }\left\langle\operatorname{ker}\left({ }^{\prime} d_{1}^{1-d, 2 d-2}\right), \operatorname{ker}\left({ }^{\prime} d_{1}^{1-d, 2 d-2}\right)\right\rangle \subsetneq \mathfrak{m}, \quad \text { alors } \nu^{d-1}\left(E_{2}^{1-d, 2 d-2}\right) \subsetneq \mathfrak{m} . E_{2}^{d-1,0} .
$$

Venons-en maintenant à la situation qui nous intéresse, à savoir $\mathfrak{X}=\widehat{\Omega}_{K}^{d-1} / \Gamma$ où $\widehat{\Omega}_{K}^{d-1}$ est le modèle formel de Deligne (ou Drinfeld) de $\Omega_{K}^{d-1}$ (cf. [10]) et $\Gamma$ est un sous-groupe discret 
sans torsion de $P G L_{d}(K)$. Lorsque $\Gamma=\{1\}$, on sait que $\widehat{\Omega}_{K}^{d-1}$ est fortement semi-stable au sens de 4.3.1. L'ensemble $I$ des composantes irréductibles est en bijection avec l'ensemble $B T$ des sommets de l'immeuble de Bruhat-Tits de $P G L_{d}(K)$, et la structure simpliciale $B T^{(\bullet)}$ qui en découle est celle de Bruhat-Tits. Par exemple, $B T^{(d)}$ est l'ensemble des chambres de l'immeuble et $B T^{(d-1)}$ celui des murs (facettes de codimension 1). En général, pour que $\mathfrak{X}=\widehat{\Omega}_{K}^{d-1} / \Gamma$ soit fortement semi-stable, il faut et il suffit que l'étoile d'un sommet soit contenue dans un domaine fondamental de $\Gamma$ dans $B T^{(\bullet)}$ (sinon il y a des points doubles). On a alors $I^{(m)}=\Gamma \backslash B T^{(m)}$. Nous fixons une orientation $S L_{d}(K)$-équivariante de $B T^{(\bullet)}$ de la manière suivante : on choisit d'abord un ordre sur les sommets d'une chambre $\Delta$ et on le transporte à toute autre chambre par l'action transitive de $S L_{d}(K)$, sans ambiguïté puisque le stabilisateur et le fixateur de $\Delta$ coïncident dans $S L_{d}(K)$; on obtient donc une orientation sur chaque simplexe maximal et on vérifie que ces orientations se recollent bien le long des murs. L'orientation de $B T^{(\bullet)}$ obtenue est équivariante par tout $\Gamma$ discret sans torsion et induit donc une orientation de $\Gamma \backslash B T^{(\bullet)}$.

Fixons un tore maximal $T$, notons $A$ l'ensemble des sommets de l'appartement de $B T$ associé, et $A^{(\bullet)}$ l'ensemble simplicial engendré par $A$. Fixons aussi une chambre $\Delta$ dans $A^{(d)}$. Nous noterons $A_{+}^{(d)}$, resp. $A_{-}^{(d)}$, l'ensemble des chambres de $A^{(d)}$ qui sont à distance paire, resp. impaire de $\Delta$.

4.3.8. LEMME. - Soit $\Gamma$ un sous-groupe discret sans torsion et cocompact de $T$ tel que le quotient $\widehat{\Omega}_{K}^{d-1} / \Gamma$ soit fortement semi-stable. Alors les fonctions caractéristiques $1_{A_{ \pm}^{(d)}}$ de $A_{ \pm}^{(d)}$ dans $B T^{(d)}$ définissent deux éléments de ${ }^{\prime} E_{1}^{1-d, 2 d-2}$ tels que,

(i) avec notre choix d'orientation, $1_{A_{+}^{(d)}}-1_{A_{-}^{(d)}} \in \operatorname{ker}^{\prime} d_{1}^{1-d, 2 d-2}$

(ii) $\left\langle\left(1_{A_{+}^{(d)}}-1_{A_{-}^{(d)}}\right),\left(1_{A_{+}^{(d)}}-1_{A_{-}^{(d)}}\right)\right\rangle=\left|\Gamma \backslash A^{(d)}\right|$.

Preuve. - Le fait que ces fonctions caractéristiques induisent des éléments de $E_{1}^{1-d, 2 d-2}=$ $\Lambda\left[\Gamma \backslash B T^{(d)}\right]$ découle de

- $A_{+}^{(d)}$ et $A_{-}^{(d)}$ sont stables par $\Gamma$ puisque un domaine fondamental de $\Gamma$ contient l'étoile d'un sommet.

- $\Gamma$ a un nombre fini d'orbites dans $A^{(d)}$ puisqu'il est cocompact dans $T$.

Montrons maintenant le point (i). Soit $M$ un mur de $B T^{(d-1)}$. L'ensemble des chambres de $\Gamma \backslash A^{(d-1)}$ contenant $\Gamma . M$ est non vide seulement si $M \in A^{(d-1)}$, auquel cas il contient deux éléments, $\Gamma \Delta_{+}$et $\Gamma \Delta_{-}$où $\Delta_{+}$et $\Delta_{-}$sont les chambres mitoyennes de $A^{(d)}$ contenant $M$, la première paire et la seconde impaire. On a alors

$$
{ }^{\prime} d_{1}^{1-d, 2 d-2}\left(1_{A_{+}^{(d)}}-1_{A_{-}^{(d)}}\right)(M)=\varepsilon_{\Gamma \Delta_{+}, \Gamma \cdot M}-\varepsilon_{\Gamma \Delta_{-}, \Gamma \cdot M} .
$$

Mais par définition de notre orientation, puisque $\Delta_{+}$se déduit de $\Delta_{-}$par un élément de $S L_{d}(K)$ laissant fixe le mur mitoyen, on a $\varepsilon_{\Gamma \Delta_{+}, \Gamma . M}=\varepsilon_{\Delta_{+}, M}=\varepsilon_{\Delta_{-}, M}=\varepsilon_{\Gamma \Delta_{-}, \Gamma . M}$.

Le point (ii) est immédiat, vue la définition de la forme bilinéaire $\langle\cdot, \cdot\rangle \operatorname{sur}^{\prime} E_{1}^{1-d, 2 d-2}$.

Remarquons qu'il est facile de choisir $\Gamma$ tel que $\left|\Gamma \backslash A^{(d)}\right|$ soit inversible dans $\Lambda$. C'est par exemple le cas pour le réseau engendré par les $\alpha^{\vee}\left(\varpi_{K}\right)$ où $\alpha^{\vee}$ parcourt les racines simples de $T$ : dans ce cas on a en effet $\left|\Gamma \backslash A^{(d)}\right|=\left|W_{G}\right|=d$ ! qui est inversible dans l'anneau banal $\Lambda$.

Pour appliquer les deux lemmes précédents à notre problème, il nous faut montrer la dégéneresence de la suite spectrale.

4.3.9. Lemme (On rappelle que $\Lambda$ est en particulier banal pour $\left.P G L_{d}(K)\right)$. - Pour tout $\Gamma$ discret sans torsion dans $P G L_{d}(K)$, la suite spectrale de Rapoport-Zink de la proposition 4.3.2 associée à $\mathfrak{X}=\widehat{\Omega}_{K}^{d-1} / \Gamma$ dégénère en $E_{2}$.

4 SÉRIE - TOME $39-2006-\mathrm{N}^{\circ} 1$ 
Preuve. - Dans le cas où $\Lambda$ est une extension de $\mathbb{Q}_{l}$, la dégénérescence est vraie pour tout $\mathfrak{X}$, grâce au théorème de pureté des $H^{*}\left(Y_{I}^{\text {ca }}, \mathbb{Q}_{l}\right)$ de Deligne et a un argument de poids classique. Pour un $\mathfrak{X}$ général, il n'y a pas de théorie des poids «à valeurs dans $\Lambda$ », mais dans notre cas les variétés $Y_{J}$ ont une cohomologie particulièrement simple : nous allons expliquer (rappeler) pourquoi pour tout $J$, on a

(i) $H^{*}\left(Y_{J}^{\text {ca }}, \Lambda\right)$ est non nul seulement si $* \in 2 \mathbb{N}$ et $0 \leqslant * \leqslant 2 d-2|J|$.

(ii) Le Frobenius géométrique $\sigma$ agit sur $H^{2 i}\left(Y_{J}^{\mathrm{ca}}, \Lambda\right)$ par multiplication par $q^{i}$.

À partir de là, la dégénérescence est une conséquence de l'argument de poids habituel, compte tenu du fait que pour un anneau banal et pour $0 \leqslant i<j \leqslant d-1$, on a $\Lambda[X]=\left(X-q^{i}\right) \Lambda[X]+$ $\left(X-q^{j}\right) \Lambda[X]$.

Rappelons tout d'abord la structure de $Y_{J}$ : soit $B^{n}$ la $k$-variété obtenue en éclatant $\mathbb{P}^{n}$ le long du produit des (idéaux définissant ses) sous-variétés linéaires $k$-rationnelles. Alors pour tout $J$, les composantes connexes de $Y_{J}$ sont de la forme $B^{d_{1}} \times \cdots \times B^{d_{|J|}}$ avec $d_{1}+\cdots+d_{|J|}=d-|J|$, cf. [29, par. 6]. Il suffit donc de prouver les deux assertions ci-dessus pour les variétés $B^{n}$. Mais on peut obtenir ces variétés selon la suite d'éclatements plus agréables suivante (cf. [29, par. 4]) :

$$
B^{n}=Y_{n-1} \stackrel{\psi_{n-1}}{\longrightarrow} Y_{n-2} \rightarrow \cdots \rightarrow Y_{1} \stackrel{\psi_{1}}{\longrightarrow} Y_{0}=\mathbb{P}^{n}
$$

où $Y_{m+1}$ est l'éclaté de $Y_{m}$ le long de la réunion $Z_{m}$ des transformés stricts des sous-variétés linéaires de dimension $m$ de $\mathbb{P}^{n}$ dans $Y_{m}$. L'avantage est qu'à chaque étape, la sous-variété $Z_{m}$ le long de laquelle on éclate est lisse de pure dimension $m$.

Alors d'après [41, VII, 8.5] : on a pour tout $m$ des suites exactes :

$$
0 \rightarrow H^{*+2 m-2 n}\left(Z_{m}^{\mathrm{ca}}, \Lambda\right) \rightarrow H^{*-2}\left(\psi_{m}^{*}\left(Z_{m}\right)^{\mathrm{ca}}, \Lambda\right) \oplus H^{*}\left(Y_{m}^{\mathrm{ca}}, \Lambda\right) \rightarrow H^{*}\left(Y_{m+1}^{\mathrm{ca}}, \Lambda\right) \rightarrow 0 .
$$

Or, par construction, les composantes connexes de $Z_{m}$ sont toutes isomorphes à $B^{m}$. De plus, $\psi_{m}: \psi_{m}^{*}\left(Z_{m}\right) \rightarrow Z_{m}$ est un $\mathbb{P}^{n-m-1}$-fibré. On montre alors les deux assertions annoncées par double récurrence sur $n$ et $m$.

Appliquons ceci à la suite spectrale de Rapoport-Zink : on obtient que $E_{1}^{-r, m+r}$ est non-nul seulement si $m+r$ est pair auquel cas l'action du Frobenius géométrique $\sigma$ sur $E_{1}^{-r, m+r}$ est la multiplication $\operatorname{par} q^{(m+r) / 2}$. Rappelons que $0 \leqslant m+r \leqslant 2 d-2$ et, sous l'hypothèse $\Lambda$ banal, on a $\Lambda[X]=\left(X-q^{i}\right) \Lambda[X]+\left(X-q^{j}\right) \Lambda[X]$ pour tous $0 \leqslant i, j \leqslant d-1$. Comme les différentielles $d_{n}^{-r, m+r}$ sont des applications $\sigma$-équivariantes $E_{n}^{-r, m+r} \rightarrow E_{n}^{-r+n, m+r-n+1}$, elles sont nulles dès que $n \geqslant 2$.

D'après les trois lemmes précédents, si $\Gamma$ est le sous-groupe discret sans torsion et cocompact de $T$ engendré par les $\alpha^{\vee}\left(\varpi_{K}\right)$, alors sur le terme $E_{\infty}=E_{2}$ de la suite spectrale de RapoportZink du quotient $\widehat{\Omega}_{K}^{d-1} / \Gamma$, on a :

$$
\nu^{d-1}\left(E_{\infty}^{1-d, 2 d-2}\right) \subsetneq \mathfrak{m} \cdot E_{\infty}^{d-1,0}
$$

D'après la dernière assertion de la proposition 4.3.2, l'action de $(\mu-1)^{d-1}$ sur $H^{d-1}\left(\Omega^{d-1, \text { ca }} / \Gamma, \Lambda\right)$ se factorise selon le diagramme

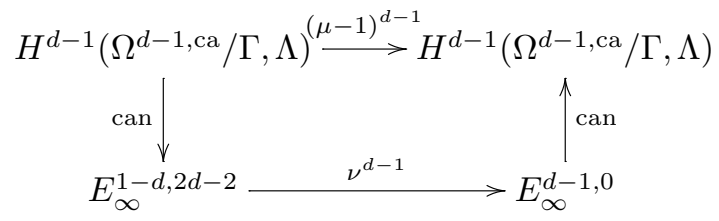


D'après la preuve du lemme précédent, le monomorphisme de droite est scindé : son image est le sous-module des invariants sous un Frobenius géométrique $\phi$ et un facteur direct est donné par $\operatorname{ker}\left(\prod_{i=1}^{d-1}\left(\phi-q^{i}\right)\right)$. Il s'ensuit que

$$
(\mu-1)^{d-1} \notin \mathfrak{m} \operatorname{End}_{\Lambda}\left(H^{d-1}\left(\Omega^{d-1, \mathrm{ca}} / \Gamma, \Lambda\right)\right) .
$$

D'après le théorème B.3.1, on en déduit que

$$
(\gamma(\mu)-1)^{d-1} \notin \mathfrak{m} \operatorname{End}_{D^{b}\left(\Lambda P G_{d}\right)}\left(R \Gamma_{c}\left(\Omega^{d-1, \mathrm{ca}}, \Lambda\right)\right) .
$$

Mais puisque $(\gamma(\mu)-1)^{d-1}=\log (\gamma(\mu))^{d-1}=(N \otimes \mu)^{d-1}$, on en déduit la proposition 4.2.7.

\subsection{Preuve du théorème}

Fixons $I \subseteq S$, et choisissons un relèvement de Frobenius $\phi$. Le scindage $\alpha_{\phi}$ de la proposition 4.2.6 induit le premier isomorphisme de $\Lambda$-modules suivant :

$$
\begin{aligned}
& R \operatorname{Hom}_{D^{b}\left(\Lambda P G_{d}\right)}\left(R \Gamma_{c}\left(\Omega_{K}^{d-1, \mathrm{ca}}, \Lambda\right), \pi_{I}^{\Lambda}\right) \\
& \quad \simeq \bigoplus_{i=0}^{d-1} R \operatorname{Hom}_{D^{b}\left(\Lambda P G_{d}\right)}\left(\pi_{\{1, \ldots, i\}}^{\Lambda}(-i), \pi_{I}^{\Lambda}\right)[d-1+i] \\
& \simeq \bigoplus_{i=0}^{d-1} \operatorname{Ext}_{P G_{d}}^{\delta(i, I)}\left(\pi_{\{1, \ldots, i\}}^{\Lambda}, \pi_{I}^{\Lambda}\right)(i)[d-1+i-\delta(i, I)]
\end{aligned}
$$

et le second est une conséquence de 2.1.4(i); on y a posé $\delta(i, I):=\delta(\{1, \ldots, i\}, I)$. De plus, par définition de $\alpha_{\phi}$, ces isomorphismes sont $\phi$-équivariants. Choisissons pour chaque $\Lambda$-droite $\operatorname{Ext}_{P G_{d}}^{\delta(i, I)}\left(\pi_{\{1, \ldots, i\}}^{\Lambda}, \pi_{I}^{\Lambda}\right)$ un générateur $e_{i}$, de manière à simplifier un peu l'écriture en :

$$
R \operatorname{Hom}_{D^{b}\left(\Lambda P G_{d}\right)}\left(R \Gamma_{c}\left(\Omega_{K}^{d-1, \mathrm{ca}}, \Lambda\right), \pi_{I}^{\Lambda}\right)[1-d] \simeq \bigoplus_{i=0}^{d-1} e_{i} \otimes \Lambda(i)[i-\delta(i, I)] .
$$

L'action de $N$ (à droite) est donnée par U-produit par l'élément

$$
\alpha_{\phi, *}(N)=\sum_{i=0}^{d-1} \beta_{i, i+1} \in \bigoplus_{i=0}^{d-1} \operatorname{Ext}_{P G_{d}}^{1}\left(\pi_{\{1, \ldots, i+1\}}^{\Lambda}, \pi_{\{1, \ldots, i\}}^{\Lambda}\right)
$$

où les $\beta_{i, i+1}$ sont le système de générateurs (4.2.4) associé à l'isomorphisme $\beta_{\phi}$ de la proposition 4.2.6. On a donc pour tout $i=0, \ldots, d-1$

$$
N . e_{i}=e_{i} \cup \alpha_{\phi, *}(N)=e_{i} \cup \beta_{i, i+1} \in \Lambda e_{i+1} .
$$

La formule 2.1.4(ii) nous montre alors que $N . e_{i} \in \Lambda^{\times} e_{i+1}$ si on a l'additivité des $\delta$ suivante :

$$
\delta(i+1, I)=\delta(i, I)+\delta(\{1, \ldots, i\},\{1, \ldots, i+1\})=\delta(i, I)+1,
$$

et est nul sinon. L'additivité des $\delta$ peut encore s'écrire :

$$
i+1-\delta(i+1, I)=i-\delta(i, I) .
$$

$4^{\mathrm{e}}$ SÉRIE - TOME $39-2006-\mathrm{N}^{\circ} 1$ 
4.4.1. Lemme. - La fonction $i \in\{0, \ldots, d-1\} \mapsto \partial(i):=i-\delta(i, I)$ croît de $\partial(0)=-|I| \grave{a}$ $\partial(d-1)=|I|$. Ses sauts non-nuls sont égaux à 2 . Pour tout $0 \leqslant k \leqslant|I|$, on a

$$
\partial^{-1}(-|I|+2 k)=\left\{i_{k}, \ldots, i_{k+1}-1\right\}
$$

où $I=\left\{i_{1}, \ldots, i_{|I|}\right\}$ et on a posé $i_{0}:=0$ et $i_{|I|+1}:=d$.

Preuve. - Élémentaire.

Le lemme permet de réécrire

$$
R \operatorname{Hom}_{D^{b}\left(\Lambda P G_{d}\right)}\left(R \Gamma_{c}\left(\Omega_{K}^{d-1, \mathrm{ca}}, \Lambda\right), \pi_{I}^{\Lambda}\right)[1-d] \simeq \bigoplus_{k=0}^{|I|}\left(\bigoplus_{i=i_{k}}^{i_{k+1}-1} e_{i} \otimes \Lambda(i)\right)[-|I|+2 k]
$$

et la discussion qui le précède montre que l'action de $N$ se fait degré par degré, et que pour un degré $-|I|+2 k$ fixé et pour $i_{k}<j<i_{k+1}$, elle induit un isomorphisme $e_{j-1} \otimes \Lambda(j-1) \stackrel{\sim}{\longrightarrow}$ $e_{j} \otimes \Lambda(j)$. Ceci achève la preuve du théorème 4.1.5.

\subsection{Application aux variétés uniformisées par $\Omega_{K}^{d-1}$}

Soit $\Gamma$ un sous-groupe discret sans torsion et cocompact dans $P G_{d}$. On sait depuis Mustafin [32] que le quotient analytique $\Omega_{K}^{d-1} / \Gamma$ s'identifie alors à l'analytification d'une variété propre et lisse sur $K$. Par les théorèmes de type GAGA de Berkovich [2, 7.1], on peut identifier la cohomologie du quotient analytique avec celle de la variété algébrique correspondante. Les espaces de cohomologie $l$-adique $H^{p}\left(\Omega_{K}^{d-1, \text { ca }} / \Gamma, \overline{\mathbb{Q}}_{l}\right)$ sont donc des représentations de dimension finie $l$-adiques continues de $W_{K}$. Leurs semi-simplifiées ont été calculées par Schneider et Stuhler dans [38]. L'action continue de $W_{K}$ munit chacun d'eux d'un opérateur nilpotent, par le théorème de «la monodromie unipotente» de Grothendieck. Ces opérateurs de monodromie sont décrits par la conjecture «monodromie-poids » démontrée par Ito [29] dans le cas qui nous intéresse ici : on a donc une description des espaces de cohomologie à Frobeniussemi-simplification près.

Notre étude du complexe de cohomologie permet bien sûr de retrouver la description de Schneider-Stuhler, mais fournit aussi une preuve très différente de celle d'Ito de la conjecture Monodromie-Poids, et montre aussi que tout relèvement de Frobenius agit de manière semisimple.

4.5.1. COROLLAIRE. - Soit $\Gamma$ un sous-groupe discret cocompact et sans torsion de $P G L_{d}(K)$ et $m_{S t}$ la multiplicité de la représentation de Steinberg dans la représentation admissible $\overline{\mathbb{Q}}_{l}^{\infty}\left[P G_{d} / \Gamma\right]$. Alors on a un isomorphisme $W_{K}$-équivariant dans $D^{b}\left(\overline{\mathbb{Q}}_{l}\right)$ :

$$
R \Gamma\left(\Omega_{K}^{d-1, \mathrm{ca}} / \Gamma, \overline{\mathbb{Q}}_{l}\right) \simeq\left(\bigoplus_{i=0}^{d-1} \overline{\mathbb{Q}}_{l}(-i)[-2 i]\right) \oplus\left(S p_{d}^{\overline{\mathbb{Q}}_{l}}\right)^{m_{S t}}(1-d)[1-d]
$$

En particulier, la conjecture Monodromie-Poids est satisfaite par les $H^{i}\left(\Omega_{K}^{d-1, \mathrm{ca}} / \Gamma, \overline{\mathbb{Q}}_{l}\right)$ et l'action d'un relèvement de Frobenius y est semi-simple.

Preuve. - Si $V$ est une $\overline{\mathbb{Q}}_{l}$-représentation lisse de $P G_{d}$, on a des isomorphismes canoniques

$$
\operatorname{Hom}_{P G_{d}}\left(V, \overline{\mathbb{Q}}_{l}^{\infty}\left[P G_{d} / \Gamma\right]\right) \stackrel{\sim}{\longrightarrow} \operatorname{Hom}_{\Gamma}\left(V, \overline{\mathbb{Q}}_{l}\right) \stackrel{\sim}{\longrightarrow}\left(V \otimes_{\overline{\mathbb{Q}}_{l}[\Gamma]} \overline{\mathbb{Q}}_{l}\right)^{*}
$$


où l'étoile désigne le dual d'un $\overline{\mathbb{Q}}_{l}$-espaces vectoriel. Comme la restriction d'une représentation lisse projective de $P G_{d}$ à $\Gamma$ est encore projective, et comme le passage au dual est un foncteur exact, on peut dériver ces isomorphimes pour en déduire :

$$
R \operatorname{Hom}_{D^{b}\left(\overline{\mathbb{Q}}_{l} P G_{d}\right)}\left(R \Gamma_{c}\left(\Omega_{K}^{d-1, \mathrm{ca}}, \overline{\mathbb{Q}}_{l}\right), \overline{\mathbb{Q}}_{l}^{\infty}\left[P G_{d} / \Gamma\right]\right) \stackrel{\sim}{\longrightarrow}\left(R \Gamma_{c}\left(\Omega_{K}^{d-1, \mathrm{ca}}, \overline{\mathbb{Q}}_{l}\right) \otimes_{\overline{\mathbb{Q}}_{l}[\Gamma]}^{L} \overline{\mathbb{Q}}_{l}\right)^{*} .
$$

D'après (4.1.4), le membre de droite est $W_{K}$-isomorphe à $R \Gamma\left(\Omega_{K}^{d-1, \text { ca }} / \Gamma, \overline{\mathbb{Q}}_{l}\right)^{*}$. Par ailleurs, la représentation admissible $\overline{\mathbb{Q}}_{l}^{\infty}\left[P G_{d} / \Gamma\right]$ de $P G_{d}$ est semi-simple et ses constituants sont unitarisables. Les seules représentations elliptiques unitarisables sont la triviale et la Steinberg. La première apparaît avec multiplicité 1 dans $\overline{\mathbb{Q}}_{l}^{\infty}\left[P G_{d} / \Gamma\right]$. Il ne reste plus qu'à appliquer le théorème 4.1 .5 en tenant compte du passage au dual; on obtient la formule annoncée, et on vérifie facilement que la conjecture Monodromie-Poids est satisfaite.

\section{Remerciements}

Je remercie M. Harris pour les nombreux échanges à propos du contenu de ce texte et V. Berkovich de m'avoir permis d'utiliser ses notes non-publiées. J'ai aussi été aidé au cours de ma rédaction par de nombreuses conversations avec P. Boyer, L. Fargues, G. Henniart, B.C. Ngô, S. Orlik et M. Strauch que je remercie tous. Les idées principales de ce texte sont apparues au cours de longues discussions avec Alain Genestier qui m'a initié aux espaces de Drinfeld. Sans lui, pas une ligne n'aurait été écrite. Ces discussions ont été permises par l'environnement exceptionnel de l'IHÉS ; je remercie cet institut pour sa longue hospitalité et Laurent Lafforgue pour m'y avoir invité en compagnie de ceux que je viens de remercier.

\section{Appendice A. Sorites sur les complexes}

\section{A.1. Critères de scindage d'un complexe}

Le but de cette section est de donner des critères de scindage d'un complexe cohomologiquement borné d'une catégorie dérivée. Ces critères sont très simples et certainement bien connus des spécialistes-comme l'a signalé G. Laumon à l'auteur, on trouve déja dans Deligne [15] un énoncé semblable à A.1.4 ci-dessous, mais un peu trop différent pour être cité tel quel. Le cadre naturel, un peu plus général, est celui des $t$-catégories.

A.1.1. Soit $\mathcal{D}$ une catégorie triangulée munie d'une $t$-structure $\left(\mathcal{D}^{\leqslant 0}, \mathcal{D} \geqslant 0\right)$ non-dégénérée, cf. [1, 1.3.7.]. On utilise les notations habituelles : $\tau_{\leqslant q}$ et $\tau_{\geqslant q}$ pour les endofoncteurs de troncation et $\mathcal{H}^{q}:=\tau_{\leqslant q} \tau_{\geqslant q}[q]=\tau_{\geqslant q} \tau_{\leqslant q}[q]=\tau_{\geqslant 0} \tau_{\leqslant 0} \circ[q]$ les (endo)foncteurs cohomologiques. On note aussi $\mathcal{D}^{+}$, resp. $\mathcal{D}^{-}$et $\mathcal{D}^{b}$, les sous-catégories triangulées formées des objets $X$ tels que $\mathcal{H}^{q}(X)=0$ pour $q \ll 0$, resp. $q \gg 0$, resp. $q \ll 0$ ou $q \gg 0$ (dans ce dernier cas $X$ est dit «cohomologiquement borné»).

Nous dirons qu'un objet $X$ est scindable, s'il est cohomologiquement borné et s'il existe un isomorphisme dans $\mathcal{D}$

$$
\alpha: X \stackrel{\sim}{\longrightarrow} \bigoplus_{q} \mathcal{H}^{q}(X)[-q] .
$$

Un tel isomorphisme sera appelé un scindage s'il induit l'identité en cohomologie, c'est-à-dire si pour tout $q \in \mathbb{Z}$, on a $\mathcal{H}^{q}(\alpha)=\operatorname{Id}_{\mathcal{H}^{q}(X)}$.

A.1.2. Lemme. - Soit $X \in D^{-}$. Fixons $q \in \mathbb{Z}$ et supposons que $\operatorname{Hom}_{\mathcal{D}}\left(\mathcal{H}^{l}(X)[k], \mathcal{H}^{q}(X)\right)=0$ pour tout couple d'entiers $(l, k)$ tel que $l+k=q-1$. Alors

$4^{\text {e }}$ SÉRIE - TOME $39-2006-\mathrm{N}^{\circ} 1$ 


\section{l'application}

$$
\mathcal{H}^{q}: \operatorname{Hom}_{\mathcal{D}}\left(X, \mathcal{H}^{q}(X)[-q]\right) \rightarrow \operatorname{Hom}_{\mathcal{D}}\left(\mathcal{H}^{q}(X), \mathcal{H}^{q}(X)\right)
$$

est surjective.

Preuve. - Quitte à décaler, on peut supposer que $q=0$, ce que nous ferons. L'application en question s'inscrit dans un diagramme

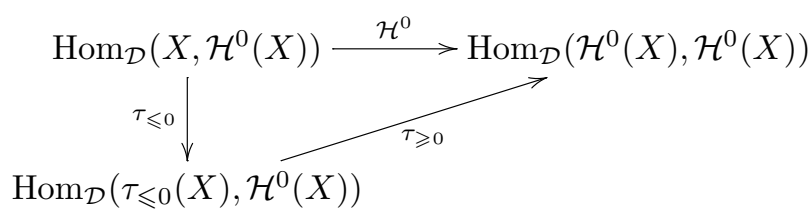

où la flèche notée $\tau_{\geqslant 0}$ est bijective et la flèche notée $\tau_{\leqslant 0}$ est la composition avec le morphisme canonique $\tau_{\leqslant 0}(X) \rightarrow X$. Ainsi, l'application de l'énoncé est surjective si et seulement si pour tout morphisme $\gamma: \tau_{\leqslant 0}(X) \rightarrow \mathcal{H}^{0}(X)$, on peut compléter le diagramme suivant

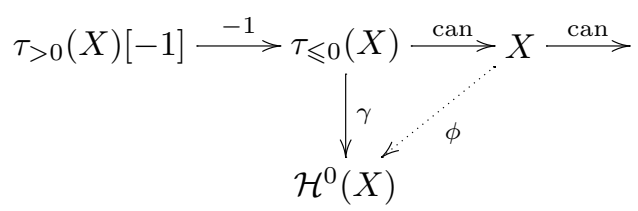

Une condition nécessaire est bien sûr que $\gamma \circ-1=0$, et l'axiome de l'octaèdre montre que cette condition est suffisante. Nous allons en fait montrer par récurrence sur l'entier $n \in \mathbb{Z}$ tel que $X \in \mathcal{D}^{\leqslant n}$ que l'hypothèse de l'énoncé (c'est-à-dire : $\operatorname{Hom}_{\mathcal{D}}\left(\mathcal{H}^{l}(X)[k], \mathcal{H}^{0}(X)\right)=0$ pour tout couple d'entiers $(l, k)$ tel que $l+k=-1)$ implique

$$
\operatorname{Hom}_{\mathcal{D}}\left(\tau_{>0}(X)[-1], \mathcal{H}^{0}(X)\right)=0,
$$

ce qui sera suffisant pour prouver le lemme.

Remarquons que si $n \leqslant 0$, on a $\tau_{>0}(X)=0$ et la propriété cherchée est immédiate. Supposons donc $n>0$ et la propriété montrée pour $n-1$. Soit $X \in \mathcal{D}^{\leqslant n}$. L'objet $\tau_{<n}(X)$ est dans $\mathcal{D}^{\leqslant n-1}$ et vérifie l'hypothèse du lemme, on peut donc lui appliquer l'hypothèse de récurrence. Le triangle distingué $\tau_{>0} \tau_{<n}(X) \rightarrow \tau_{>0}(X) \rightarrow \mathcal{H}^{n}(X)[-n]$ fournit une suite exacte

$$
\begin{aligned}
\operatorname{Hom}_{\mathcal{D}}\left(\mathcal{H}^{n}(X)[-1-n], \mathcal{H}^{0}(X)\right) & \rightarrow \operatorname{Hom}_{\mathcal{D}}\left(\tau_{>0}(X)[-1], \mathcal{H}^{0}(X)\right) \\
& \rightarrow \operatorname{Hom}_{\mathcal{D}}\left(\tau_{>0} \tau_{<n}(X)[-1], \mathcal{H}^{0}(X)\right) .
\end{aligned}
$$

Le premier terme est nul par hypothèse, ainsi que le dernier par hypothèse de récurrence (remarquer que la flèche canonique $\mathcal{H}^{0}\left(\tau_{<n}(X)\right) \rightarrow \mathcal{H}^{0}(X)$ est un isomorphisme), le terme du milieu est donc nul aussi.

A.1.3. Corollaire. - Soit $X \in \mathcal{D}$ cohomologiquement borné. Supposons que

$$
\operatorname{Hom}_{\mathcal{D}}\left(\mathcal{H}^{l}(X)[k], \mathcal{H}^{q}(X)\right)=0
$$

pour tout triplet d'entiers $(q, l, k)$ tel que $l+k=q-1$. Alors $X$ est scindable. 
Preuve. - D'après le lemme précédent, pour tout $q \in \mathbb{Z}$ on peut trouver $\alpha_{q}: X \rightarrow \mathcal{H}^{q}(X)[-q]$ tel que $\mathcal{H}^{q}\left(\alpha_{q}\right)=\operatorname{Id}_{\mathcal{H}^{q}(X)}$. Le morphisme somme

$$
\bigoplus_{q} \alpha_{q}: X \rightarrow \bigoplus_{q} \mathcal{H}^{q}(X)[-q]
$$

induit l'identité en cohomologie et par conséquent est un isomorphisme, car la $t$-structure est non-dégénérée.

Dans le lemme suivant on suppose de plus que $\mathcal{D}$ est $R$-linéaire pour un anneau commutatif $R$ fixé.

A.1.4. Lemme. - Soit $X$ un objet cohomologiquement borné de $\mathcal{D}$ et $\phi \in \operatorname{End}_{\mathcal{D}}(X)$. On suppose donnée une famille de polynômes $P_{q}(T) \in R[T], q \in \mathbb{Z}$, presque tous égaux à 1 , et tels que pour tout $q \in \mathbb{Z}$, on ait $P_{q}\left(\mathcal{H}^{q}(\phi)\right)=0$ dans $\operatorname{End}_{\mathcal{D}}\left(\mathcal{H}^{q}(X)\right)$.

(i) Posons $P(T):=\prod_{q \in \mathbb{Z}} P_{q}(T)$; alors on a $P(\phi)=0$ dans $\operatorname{End}_{\mathcal{D}}(X)$.

(ii) Supposons de plus que pour tous $p \neq q$, on a $P_{q} R[X]+P_{p} R[X]=R[X]$. Alors il existe un unique scindage

$$
\alpha: X \rightarrow \bigoplus_{q} \mathcal{H}^{q}(X)[-q]
$$

$$
\text { tel que } \alpha \phi \alpha^{-1}=\bigoplus_{q} \mathcal{H}^{q}(\phi)[-q] \text {. }
$$

Preuve. - Pour le point (i) on procède par récurrence sur l'amplitude cohomologique de $X$ en utilisant le résultat auxiliaire suivant :

Soient $a \in \mathbb{Z}$ et $P_{-}(T), P_{+}(T) \in R[T]$ deux polynômes tels que $P_{-}\left(\tau_{\leqslant a}(\phi)\right)=0$ dans $\operatorname{End}_{\mathcal{D}}\left(\tau_{\leqslant a}(X)\right)$ et $P_{+}\left(\tau_{>a}(\phi)\right)=0$ dans $\operatorname{End}_{\mathcal{D}}\left(\tau_{>a}(X)\right)$. Alors $P_{-} P_{+}(\phi)=0$ dans $\operatorname{End}_{\mathcal{D}}(X)$.

Nous laisserons la récurrence au lecteur, mais nous allons montrer l'assertion ci-dessus. On définit un morphisme $\psi_{-}: \tau_{>a}(X) \rightarrow X$ par le diagramme commutatif suivant :

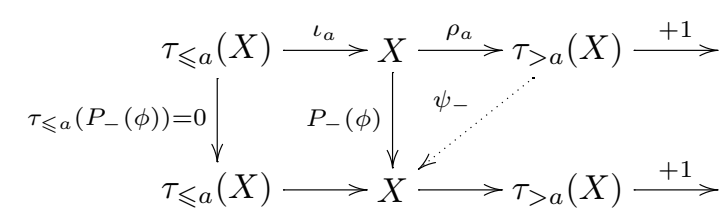

L'existence de $\psi_{-}$est assurée par la suite exacte

$$
\operatorname{Hom}_{\mathcal{D}}\left(\tau_{>a}(X), X\right) \rightarrow \operatorname{Hom}_{\mathcal{D}}(X, X) \rightarrow \operatorname{Hom}_{\mathcal{D}}\left(\tau_{\leqslant a}(X), X\right) .
$$

De même on définit $\psi_{+}: X \rightarrow \tau_{\leqslant a}(X)$ par le diagramme

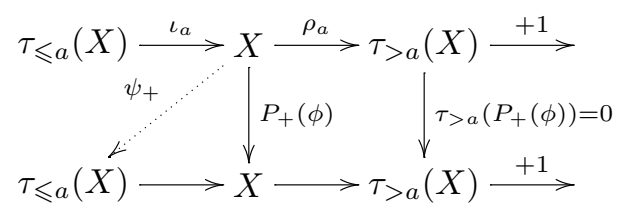

On calcule alors $\left(P_{-} P_{+}\right)(\phi)=P_{-}(\phi) \circ P_{+}(\phi)=\psi_{-} \circ \rho_{a} \circ \iota_{a} \circ \psi_{+}=0$ car $\rho_{a} \circ \iota_{a}=0$.

Pour l'existence dans le point (ii), on procède encore par récurrence sur l'amplitude cohomologique de $X$ en utilisant le résultat auxiliaire suivant :

$4^{\text {e }}$ SÉRIE - TOME $39-2006-\mathrm{N}^{\circ} 1$ 
En gardant les notations ci-dessus, supposons que $P_{-}(X) R[X]+P_{+}(X) R[X]=R[X]$. Alors il existe un isomorphisme

$$
\alpha_{a}: X \stackrel{\sim}{\sim} \tau_{\leqslant a}(X) \oplus \tau_{>a}(X)
$$

tel que $\tau_{\leqslant a}\left(\alpha_{a}\right)=\operatorname{Id}_{\tau_{\leqslant a}(X)}, \tau_{>a}\left(\alpha_{a}\right)=\operatorname{Id}_{\tau_{>a}(X)}$ et $\alpha_{a} \phi \alpha_{a}^{-1}=\tau_{\leqslant a}(\phi) \oplus \tau_{>a}(\phi)$.

Nous laissons à nouveau la récurrence au lecteur, mais nous prouvons cette assertion. Pour cela, remarquons que quitte à remplacer $P_{-}$, resp. $P_{+}$, par un de ses multiples dans $R[X]$, on peut supposer $P_{-}(X)+P_{+}(X)=1$. Nous allons montrer que les morphismes

$$
\alpha_{a}: X \stackrel{\psi_{+} \oplus \rho_{a}}{\longrightarrow} \tau_{\leqslant a}(X) \oplus \tau_{>a}(X) \quad \text { et } \quad \beta_{a}: \tau_{\leqslant a}(X) \oplus \tau_{>a}(X) \stackrel{\iota_{a} \oplus \psi_{-}}{\longrightarrow} X
$$

sont inverses l'un de l'autre. On a d'abord $\beta_{a} \circ \alpha_{a}=\psi_{-} \rho_{a}+\iota_{a} \psi_{+}=P_{-}(\phi)+P_{+}(\phi)=\operatorname{Id}_{X}$. Par ailleurs $\alpha_{a} \circ \beta_{a}=\psi_{+} \iota_{a} \oplus \rho_{a} \psi_{-}$. Montrons que $\rho_{a} \psi_{-}=\operatorname{Id}_{\tau_{>a}(X)}$, le raisonnement sera identique et omis pour $\psi_{+} \iota_{a}$. Tout d'abord, puisque $\tau_{>a}\left(P_{+}(\phi)\right)=0$, on a $\tau_{>a}\left(P_{-}(\phi)\right)=$ $\operatorname{Id}_{\tau_{>a}(X)}$. Il nous suffira donc de montrer que le triangle du bas du diagramme suivant est commutatif

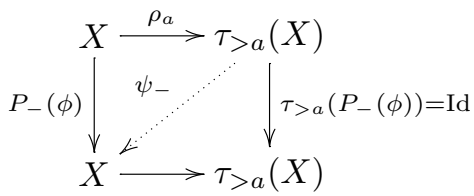

sachant que celui du haut l'est, par définition. La commutativité de celui du haut et du carré extérieur donne l'égalité $\rho_{a} \psi_{-} \rho_{a}=\tau_{>a}\left(P_{-}(\phi)\right) \circ \rho_{a}$ dans $\operatorname{Hom}_{\mathcal{D}}\left(X, \tau_{>a}(X)\right)$. En appliquant le foncteur $\tau_{>a}$ et en tenant compte de ce que $\tau_{>a}\left(\rho_{a}\right)=\operatorname{Id}_{\tau_{>a}(X)}$ (en identifiant $\tau_{>a}\left(\tau_{>a}(X)\right)$ et $\left.\tau_{>a}(X)\right)$ et de ce que $\tau_{>a}\left(\rho_{a} \psi_{-}\right)=\rho_{a} \psi_{-}$, on obtient l'égalité cherchée $\rho_{a} \psi=\tau_{>a}\left(P_{-}(\phi)\right)$.

L'isomorphisme $\alpha_{a}$ ainsi construit vérifie bien $\tau_{\leqslant a}\left(\alpha_{a}\right)=\operatorname{Id}_{\tau_{\leqslant a}(X)}$ et $\tau_{>a}\left(\alpha_{a}\right)=\operatorname{Id}_{\tau_{>a}(X)}$. On calcule aussi $\alpha_{a} \phi \beta_{a}=\rho_{a} \phi \psi_{-} \oplus \psi_{+} \phi \iota_{a}$. Or, $\rho_{a} \phi \psi_{-}=\tau_{>a}\left(\phi \psi_{-} \rho_{a}\right)=$ $\tau_{>a}(\phi) \tau_{>a}\left(P_{-}(\phi)\right)=\tau_{>a}(\phi)$ et de même on calcule $\psi_{+} \phi \iota_{a}=\tau_{\leqslant a}(\phi)$.

Il reste à voir l'unicité de $\alpha$ dans le point (ii). Soit $\beta$ un second isomorphisme vérifiant les propriétés requises par le point (ii). L'automorphisme $\beta \alpha^{-1}$ de $\bigoplus \mathcal{H}^{q}(X)[-q]$ commute à l'endomorphisme $H(\phi):=\bigoplus \mathcal{H}^{q}(\phi)[-q]$. Soit $S \subset \mathbb{Z}$ le support cohomologique de $X$. Fixons des polynômes $\left(Q_{q}\right)_{q \in S}$ tels que

$$
Q_{q} \in \prod_{p \neq q} P_{p} \cdot R[X] \quad \text { et } \quad \sum_{q \in S} Q_{q}=1 .
$$

Alors l'endomorphisme $Q_{q}(H(\phi))$ est nul sur les $\mathcal{H}^{p}(X)[-p], p \neq q$ et envoie $\mathcal{H}^{q}(X)[-q]$ identiquement dans lui-même. Comme l'automorphisme $\beta \alpha^{-1}$ commute aux $Q_{q}(H(\phi))$, il est de la forme $\bigoplus_{q} \gamma_{q}[q]$ avec $\gamma_{q} \in \operatorname{End}_{\mathcal{D}}\left(\mathcal{H}^{q}(X)\right)$. Mais comme il doit aussi induire l'identité en cohomologie on a $\gamma_{q}=\operatorname{Id}_{\mathcal{H}^{q}(X)}$ et par suite $\beta \alpha^{-1}=\mathrm{Id}$.

A.1.5. Remarque. - La preuve du point (i) montre plus précisément que si on se donne deux endomorphismes $\phi_{-}, \phi_{+}$de $X$ tels que $\tau_{\leqslant a}\left(\phi_{-}\right)=0$ et $\tau_{>a}\left(\phi_{+}\right)=0$, alors la composée $\phi_{-} \phi_{+}$est nulle dans $\operatorname{End}_{\mathcal{D}}(X)$. (Mais pas nécessairement celle dans l'autre sens !)

A.1.6. Remarque. - Toujours la même preuve montre encore que si $\phi \in \operatorname{Hom}_{\mathcal{D}}(Y, X)$ est un morphisme tel que $\tau_{\leqslant a}(\phi)=0$, resp. $\tau_{>a}(\phi)=0$, alors $\phi$ se factorise par la flèche en pointillés 
du diagramme

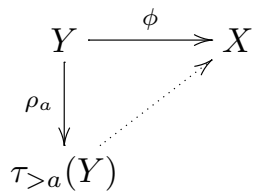

resp.

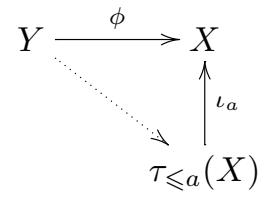

\section{A.2. Endomorphismes cohomologiquement triviaux}

On reprend le contexte précédent. Si $X \in \mathcal{D}^{b}$, on définit

$$
\mathcal{N}(X):=\operatorname{ker}\left(\operatorname{End}_{\mathcal{D}}(X) \rightarrow \prod_{q \in \mathbb{Z}} \operatorname{End}_{\mathcal{D}}\left(\mathcal{H}^{q}(X)\right)\right) .
$$

D'après le point (i) de A.1.4, cet idéal bilatère de $\operatorname{End}_{\mathcal{D}}(X)$ est formé d'éléments nilpotents d'ordre au plus la longueur $n$ de l'amplitude cohomologique de $X$. Soit maintenant $\mathcal{U}(X):=1+\mathcal{N}(X)$ le groupe des automorphismes qui induisent l'identité en cohomologie.

A.2.1. Lemme. - Supposons que la catégorie triangulée $\mathcal{D}$ est $\mathbb{Z}_{l}$-linéaire et que pour tous $p, q \in \mathbb{Z}$, le $\mathbb{Z}_{l}$-module $\operatorname{Hom}_{\mathcal{D}}\left(\mathcal{H}^{p}(X)[-p], \mathcal{H}^{q}(X)[-q]\right)$ est de type fini. Alors le $\mathbb{Z}_{l}$-module $\operatorname{End}_{\mathcal{D}}(X)$ est de type fini et le groupe $\mathcal{U}(X)$ est un pro-l-groupe.

Preuve. - Les suites spectrales habituelles montrent que le $\mathbb{Z}_{l}$-module $\operatorname{End}_{\mathcal{D}}(X)$ est un sousquotient de $\bigoplus_{p, q} \operatorname{Hom}_{\mathcal{D}}\left(\mathcal{H}^{p}(X)[-p], \mathcal{H}^{q}(X)[-q]\right)$, donc la première assertion est immédiate.

Notons $A$ la $\mathbb{Z}_{l}$-algèbre $\operatorname{End}_{\mathcal{D}}(X)$. Comme $A$ est $l$-adiquement complète, l'ensemble $A_{i}:=1+l^{i} A$ est un sous-groupe normal d'indice fini de $A^{\times}$et le morphisme canonique $A^{\times} \rightarrow \lim A / A_{i}$ est un isomorphisme, ce qui montre que $A^{\times}$est un groupe profini. De plus, son sous-groupe ouvert $A_{1}$ est pro-l.

Le sous-groupe $\mathcal{U}(X) \subset A^{\times}$est manifestement fermé, donc est profini. Pour voir qu'il est pro-l, il suffit de vérifier que les éléments de $\mathcal{U}(X) / \mathcal{U}(X) \cap A_{1}$ sont d'ordre une puissance de $l$. Or si $x \in \mathcal{N}(X)$, on a $(1+x)^{l^{k}}=1+x^{l^{k}}[\bmod l]$ et $x^{l^{k}}=0$ pour $l^{k}$ plus grand que la longueur de l'amplitude cohomologique de $X$.

On peut donner une idée un peu plus précise de la structure de $\mathcal{U}(X)$. Pour un entier $i \geqslant 1$, posons plus généralement

$$
\mathcal{N}_{i}(X):=\operatorname{ker}\left(\operatorname{End}_{\mathcal{D}}(X) \rightarrow \prod_{q \in \mathbb{Z}} \operatorname{End}_{\mathcal{D}}\left(\tau_{[q, q+i-1]}(X)\right)\right),
$$

où on a noté $\tau_{[q, q+i-1]}:=\tau_{\geqslant q} \tau_{\leqslant q+i-1}=\tau_{\leqslant q+i-1} \tau_{\geqslant q}$. On obtient ainsi une suite décroissante $\mathcal{N}(X)=\mathcal{N}_{1}(X) \supset \cdots \supset \mathcal{N}_{n}(X) \supset \mathcal{N}_{n+1}(X)=0$ d'idéaux bilatères de $\operatorname{End}_{\mathcal{D}}(X)$. La remarque A.1.5 montre que pour tous $i, j \geqslant 1$ on a $\mathcal{N}_{i}(X) \circ \mathcal{N}_{j}(X) \subset \mathcal{N}_{i+j}(X)$, ce qui fait de $\mathcal{N}_{0}(X):=\operatorname{End}_{\mathcal{D}}(X)$ un anneau filtré. On en déduit aussi une suite décroissante de groupes $\mathcal{U}_{1}(X)=\mathcal{U}(X) \supset \cdots \supset \mathcal{U}_{i}(X):=1+\mathcal{N}_{i}(X) \supset \cdots$. Pour tous $i, j \geqslant 1$, on vérifie que $\left[\mathcal{U}_{i}(X), \mathcal{U}_{j}(X)\right] \subset \mathcal{U}_{i+j}(X)$ (les crochets désignent le groupe engendré par les commutateurs). En particulier, la suite est normale et les quotients successifs sont abéliens. En fait l'application évidente $\mathcal{N}_{i}(X) / \mathcal{N}_{i+1}(X) \rightarrow \mathcal{U}_{i}(X) / \mathcal{U}_{i+1}(X)$ est un isomorphisme de groupes abéliens. Lorsque la catégorie est $\mathbb{Z}_{l}$-linéaire et vérifie la condition du lemme A.2.1, l'isomorphisme est continu.

Quant à la question de déterminer plus précisément le quotient $\mathcal{N}_{i}(X) / \mathcal{N}_{i+1}(X)$, cela semble plus délicat. Pour $i=0,1,2$, on peut montrer que ce quotient s'identifie canoniquement à un 
sous-quotient de $\prod_{p-q=i} \operatorname{Hom}_{\mathcal{D}}\left(\mathcal{H}^{p}(X)[-p], \mathcal{H}^{q}(X)[-q]\right)$. (C'est trivial pour $i=0$ et cela utilise la remarque A.1.6 pour $i=1,2$.)

\section{Appendice B. Cohomologie équivariante des espaces de Berkovich}

Dans cette partie, le contexte est le suivant : on suppose qu'un groupe localement profini $G$ agit sur un espace $K$-analytique $X$, au sens de Berkovich. Celui-ci a défini une notion de continuité pour une telle action, et tout un formalisme cohomologique étale- $G$-équivariant «continu». C'est l'existence de ce formalisme qui nous pousse à utiliser les espaces de Berkovich et leur cohomologie étale plutôt que les espaces rigides, ou les espaces adiques de Huber. Il ne fait cependant aucun doute qu'un formalisme similaire existe dans le cadre des espaces de Huber.

Lorsque $\Lambda$ est un anneau de torsion première à $p$, le formalisme en question fournit, entre autres, un complexe canonique de la catégorie dérivée bornée des $\Lambda G$-modules lisses dont la cohomologie est la cohomologie étale de $X$ à coefficients dans $\Lambda$, munie de son action canonique de $G$.

Le but de cette partie est d'exposer ce formalisme en grande partie non-publié-bien que déja utilisé dans $[20,21,23]$ — puis d'en donner une variante $l$-adique dans un langage inspiré de la cohomologie étale continue de Jannsen $[30,27,19]$, et enfin d'interpréter certaines suites spectrales de type Hochschild-Serre construites par Fargues dans [19] comme des égalités de complexes de cohomologie dans certaines catégories dérivées.

\section{B.1. Coefficients de torsion}

Cette section est un bref exposé d'un manuscrit non publié de Berkovich [5]. Les imprécisions et erreurs éventuelles ci-dessous sont de la seule responsabilité de l'auteur de ces lignes.

\section{B.1.1. Une topologie sur $\operatorname{Aut}(X)$}

Soit $X$ un espace $K$-analytique. Berkovich munit dans [3, part 6] le groupe d'automorphismes analytiques $\operatorname{Aut}(X)$ de $X$ d'une certaine topologie totalement discontinue. Par définition de cette topologie, tout ouvert distingué de $X$ (i.e. qui est différence de deux domaines analytiques compacts de $X)$ est stabilisé par un sous-groupe ouvert de $\operatorname{Aut}(X)$. L'idée maîtresse de Berkovich dans ce contexte est que tout ouvert étale "distingué » est aussi «stabilisé » par un sous-groupe ouvert de $\operatorname{Aut}(X)$. Tentons d'expliquer ce que cela signifie.

Par définition, un ouvert étale (au sens de [2]) $(U, f)$ est dit distingué s'il peut se factoriser $U \stackrel{i}{\rightarrow} \bar{U} \stackrel{f_{\bar{U}}}{\rightarrow} X$ où $\bar{U}$ est quasi-étale (au sens de [3]) et compact et $i$ fait de $U$ un ouvert distingué de $\bar{U}$. Alors d'après [3, Key Lemma 7.2.], pour un tel ouvert étale il existe un voisinage $\operatorname{de} \operatorname{Id}_{X}$ dans $\operatorname{Aut}(X)$ et, pour tout $\phi$ dans ce voisinage, un isomorphisme canonique $i_{\phi}:(U, f) \stackrel{\sim}{\longrightarrow} \phi^{-1}(U, f)$. En particulier, il existe un sous-groupe ouvert $\operatorname{Aut}_{U}(X) \operatorname{de} \operatorname{Aut}(X)$ et une action canonique

$$
\operatorname{Aut}_{U}(X) \stackrel{\beta_{U}}{\longrightarrow} \operatorname{Aut}(U)
$$

compatible avec $f:$ il suffit de poser $\beta_{U}(\phi):(U, f) \stackrel{i_{\phi}}{\longrightarrow} \phi^{-1}(U, f) \stackrel{\text { can }}{\longrightarrow}(U, f)$. C'est ce que nous entendons par la phrase « $\operatorname{Aut}_{U}(X)$ stabilise $(U, f) »$.

\section{B.1.3. Faisceaux étales $G$-équivariants}

Soit $X$ un espace $K$-analytique et $\widetilde{X_{e t}}$ le topos étale défini dans [2]. On se donne aussi un groupe discret $G$ agissant sur $X$ par automorphismes analytiques. Une structure $G$-équivariante sur un faisceau étale $\mathcal{F} \in \widehat{X_{\text {et }}}$ est la donnée d'une famille d'isomorphismes 
$\tau_{\mathcal{F}}(h): h_{*} \mathcal{F} \stackrel{\sim}{\longrightarrow} \mathcal{F}, h \in G$ vérifiant la condition de cocycle $\tau_{\mathcal{F}}\left(h^{\prime} h\right)=\tau_{\mathcal{F}}\left(h^{\prime}\right) \circ h_{*}^{\prime}\left(\tau_{\mathcal{F}}(h)\right)$. Le signe $=$ est légèrement abusif ici; il suppose qu'on a identifié les foncteurs $h_{*}^{\prime} \circ h_{*}$ et $\left(h^{\prime} h\right)_{*}$ qui sont seulement « canoniquement isomorphes ». Le couple $\left(\mathcal{F}, \tau_{\mathcal{F}}\right)$ est appelé faisceau (étale) $G$-équivariant sur $X$. La catégorie évidente formée par ces faisceaux $G$-équivariants est un topos que l'on note $\widetilde{X_{e t}}(G)$.

Maintenant, supposons que $G$ est un groupe topologique et que le morphisme $G \stackrel{\text { act }}{\longrightarrow} \operatorname{Aut}(X)$ est continu. $\mathrm{Si}(\mathcal{F}, \tau)$ est un faisceau $G$-équivariant, et $(U, f)$ un ouvert étale distingué, l'action $\beta_{U}$ de (B.1.2) munit $\mathcal{F}(U)$ d'une action du sous-groupe ouvert $G_{U}:=\operatorname{act}^{-1}\left(\operatorname{Aut}_{U}(X)\right)$ de $G$. Berkovich dit alors que $(\mathcal{F}, \tau)$ est discret si l'une des deux conditions équivalentes suivantes est vérifiée (voir aussi [21, Part 2]) :

- Pour tout morphisme étale $U \stackrel{f}{\rightarrow} X$ et toute section $s \in \mathcal{F}(U)$, tout point $u \in U$ admet un voisinage distingué $\mathcal{U}$ tel que le stabilisateur de $s_{\mid \mathcal{U}}$ dans $G_{\mathcal{U}}$ soit ouvert (définition qui ne dépend pas du choix de $G_{\mathcal{U}}$ ).

- Pour tout morphisme quasi-étale $\bar{U} \stackrel{f}{\rightarrow} X$ (voir [3, part 3]) avec $\bar{U}$ compact, le $G_{\bar{U}}$-ensemble $f^{*} \mathcal{F}(\bar{U})$ est discret (i.e. les stabilisateurs sont ouverts).

La terminologie «faisceau $G$-discret» de Berkovich diffère de celle de la théorie des représentations qui emploierait plutot les termes lisse ou localement constant (mais elle évite les conflits terminologiques avec la théorie des faisceaux). Lorsque la topologie de $G$ est discrète, tout faisceau $G$-équivariant est $G$-discret.

B.1.4. Convention. - Nous appellerons simplement «faisceau G-équivariant» ce que Berkovich appelle «faisceau G-équivariant G-discret», et nous appellerons "faisceau $G_{\mathrm{disc}}$-équivariant»ce qui est généralement appelé «faisceau G-équivariant».

Ces deux terminologies sont compatibles si l'on pense à $G_{\text {disc }}$ comme à « $G$ muni de la topologie discrète». Les faisceaux étales $G$-équivariants forment un topos que nous noterons $\widetilde{X_{\mathrm{et}}}(G)$. On a des morphismes de topos

$$
\widetilde{X_{\mathrm{et}}} \stackrel{\left(\omega_{X}, \text { ind }_{X}\right)}{\longrightarrow} \widetilde{X_{\mathrm{et}}}\left(G_{\mathrm{disc}}\right) \stackrel{\left(\iota_{X}, \infty_{X}\right)}{\longrightarrow} \widetilde{X_{\mathrm{et}}}(G) .
$$

Le foncteur $\omega_{X}: \widetilde{X_{\text {et }}}\left(G_{\text {disc }}\right) \rightarrow \widetilde{X_{\text {et }}}$ est l'oubli de la structure équivariante et son adjoint à droite est le foncteur d'induction qui à un faisceau $\mathcal{F}$ associe le faisceau $\prod_{g \in G} g_{*} \mathcal{F}$ muni de sa structure équivariante naturelle. Le foncteur $\iota_{X}: \widetilde{X_{\mathrm{et}}}(G) \rightarrow \widetilde{X_{\mathrm{et}}}\left(G_{\mathrm{disc}}\right)$ est le plongement pleinement fidèle «canonique» et son adjoint à droite est le foncteur de «lissification» $\infty_{X}$ qui associe à un faisceau $G_{\text {disc }}$-équivariant $\mathcal{F}$ le faisceau $\infty_{X}(\mathcal{F})$ engendré par le préfaisceau sur les ouverts étales distingués $U \mapsto \mathcal{F}(U)^{\infty}$ (où la notation $\mathcal{F}(U)^{\infty}$ représente le sous- $G_{U}$-ensemble discret maximal de $\mathcal{F}(U)$ ), muni de la structure $G$-équivariante induite par celle de $\mathcal{F}$. En particulier, pour tout ouvert étale $U \rightarrow X$ stabilisé par un sous-groupe ouvert $G_{U}$ de $G$, on a

$$
\Gamma_{!}\left(U, \infty_{X}(\mathcal{F})\right)=\Gamma_{!}(U, \mathcal{F})^{\infty}
$$

en désignant par $\Gamma_{!}$les sections à supports compacts.

Si $X \stackrel{\phi}{\longrightarrow} Y$ est un morphisme $G$-équivariant d'espaces analytiques, on a deux morphismes de topos $\widetilde{X_{\text {et }}} \stackrel{\left(\phi^{*}, \phi_{*}\right)}{\longrightarrow} \widetilde{Y_{\text {et }}}$ et $\widetilde{X_{\text {et }}}\left(G_{\text {disc }}\right) \stackrel{\left(\phi^{\text {disc }, *}, \phi_{*}^{\text {disc }}\right)}{\longrightarrow} \widetilde{Y_{\text {et }}}\left(G_{\text {disc }}\right)$ et un carré 2-commutatif $\left(\phi^{\text {disc }, *}, \phi_{*}^{\text {disc }}\right) \circ\left(\omega_{X}\right.$, ind $\left._{X}\right) \simeq\left(\omega_{Y}\right.$, ind $\left._{Y}\right) \circ\left(\phi^{*}, \phi_{*}\right)$. Par contre le foncteur $\phi_{*}^{\text {disc }}$ ne préserve pas en général le caractère discret d'une structure $G$-équivariante et on n'a donc pas de foncteur $\phi_{*}^{\infty}: \widetilde{X_{\mathrm{et}}}(G) \rightarrow \widetilde{Y_{\mathrm{et}}}(G)$ raisonnable.

$4^{\mathrm{e}}$ SÉRIE - TOME $39-2006-\mathrm{N}^{\circ} 1$ 
Cependant, une vérification élémentaire montre que le foncteur $\phi_{\text {! image directe à supports }}$ propres induit un foncteur $\phi_{!}^{\text {disc }}$ qui préserve le caractère discret d'une structure $G$-équivariante et induit donc à son tour un foncteur $\phi_{!}^{\infty}: \widetilde{X_{\mathrm{et}}}(G) \rightarrow \widetilde{Y_{\mathrm{et}}}(G)$.

Remarquons enfin que les foncteurs fibres en les points de $X$ forment un système conservatif sur chacun des trois topos $\widetilde{X_{\text {et }}}, \widetilde{X_{\text {et }}}\left(G_{\text {disc }}\right)$ et $\widetilde{X_{\text {et }}}(G)$.

\section{B.1.5. Compatibilités cohomologiques}

Fixons maintenant un anneau commutatif unitaire $\Lambda$ et, pour tout topos $\mathcal{T}$, notons $\operatorname{Mod}_{\Lambda}(\mathcal{T})$ la catégorie des $\Lambda$-modules de $\mathcal{T}$. C'est une catégorie abélienne qui possède suffisamment d'objets injectifs. On notera aussi $D_{\Lambda}^{+}(\mathcal{T})$ la catégorie dérivée des complexes bornés inférieurement de $\operatorname{Mod}_{\Lambda}(\mathcal{T})$ et $D_{\Lambda}^{b}(\mathcal{T})$ la sous-catégorie triangulée des objets cohomologiquement bornés de celle-ci.

Par exemple, les objets de $\operatorname{Mod}_{\Lambda}\left(\widetilde{X_{\text {et }}}(G)\right)$ sont les faisceaux en $\Lambda$-modules $G$-équivariants. Par la dernière remarque ci-dessus, une suite de morphismes dans $\operatorname{Mod}_{\Lambda}\left(\widetilde{X_{\text {et }}}(G)\right)$ est exacte si et seulement si elle l'est dans $\operatorname{Mod}_{\Lambda}\left(\widetilde{X_{\text {et }}}\left(G_{\text {disc }}\right)\right)$ si et seulement si la suite de morphismes de faisceaux sous-jacente l'est.

On a les foncteurs exacts à gauche «images directes à supports propres»

$$
\phi_{!}^{\infty}: \operatorname{Mod}_{\Lambda}\left(\widetilde{X_{\mathrm{et}}}(G)\right) \rightarrow \operatorname{Mod}_{\Lambda}\left(\widetilde{Y_{\mathrm{et}}}(G)\right)
$$

et «sections globales à support compact»

$$
\Gamma_{!}^{\infty}(X,-): \operatorname{Mod}_{\Lambda}\left(\widetilde{X_{\mathrm{et}}}(G)\right) \rightarrow \operatorname{Mod}_{\Lambda}(G),
$$

où $\operatorname{Mod}_{\Lambda}(G)$ désigne la catégorie des $\Lambda G$-modules lisses dont on note aussi $D_{\Lambda}^{+}(G)$ la catégorie dérivée. La question naturelle qui se pose est de savoir si ces foncteurs ont la bonne cohomologie, c'est-à-dire celle des foncteurs usuels $\phi_{!}$et $\Gamma_{\text {! }}$.

Lorsque $G$ est discret, il n'y a pas de problème : on vérifie en effet qu'un faisceau $G_{\text {disc }}$-équivariant $\mathcal{F}$ est $\phi_{!}^{\text {disc }}$-acyclique (resp. $\Gamma_{!}^{\text {disc }}$-acyclique) si et seulement si son faisceau sous-jacent $\omega_{X} \mathcal{F}$ est $\phi_{!}$-acyclique, resp. $\Gamma_{\text {! }}$-acyclique. Les isomorphismes de foncteurs $\phi_{!} \circ \omega_{X} \simeq$ $\omega_{Y} \circ \phi_{!}^{\text {disc }}$ et $\Gamma_{!}(X,-) \circ \omega_{X} \simeq \omega_{\mathrm{pt}} \circ \Gamma_{!}^{\text {disc }}(X,-)$ se dérivent donc en des isomorphismes $R \phi_{!} \circ \omega_{X} \simeq \omega_{Y} \circ R \phi_{!}^{\text {disc }}$ et $R \Gamma_{!}(X,-) \circ \omega_{X} \simeq \omega_{\mathrm{pt}} \circ R \Gamma_{!}^{\text {disc }}(X,-)$.

Dans le cas où $G$ est topologique, on a bien sûr des isomorphismes de foncteurs $\phi_{!}^{\text {disc }} \circ \iota_{X} \simeq$ $\iota_{Y} \circ \phi_{!}^{\infty}$ et $\Gamma_{!}^{\text {disc }}(X,-) \circ \iota_{X} \simeq \iota_{\mathrm{pt}} \circ \Gamma_{!}^{\text {disc }}(X,-)$, mais il n'est pas vrai en général que pour tout faisceau $G$-équivariant $\mathcal{F}$ acyclique dans $\operatorname{Mod}_{\Lambda}\left(\widetilde{X_{\text {et }}}(G)\right)$, le faisceau sous-jacent $\omega_{X} \iota_{X} \mathcal{F}$ le soit dans $\operatorname{Mod}_{\Lambda}\left(\widetilde{X_{\text {et }}}\right)$. Cependant, pour pouvoir dériver ces isomorphismes de foncteurs, il suffit de prouver que tout faisceau $G$-équivariant $\mathcal{F}$ se plonge dans un faisceau $G$-équivariant dont le faisceau sous-jacent est acyclique pour les foncteurs $\phi_{!}$et $\Gamma$ !

Pour cela, Berkovich utilise une construction de [SGA4, exp. XVII, par. 4.2] généralisant une construction bien connue de Godement dans le cadre topologique usuel. Choisissons un point géométrique au-dessus de chaque $x \in X$; les foncteurs fibres en ces points forment une famille conservative indexée par $X$ de points du topos $\widetilde{X_{\text {et }}}$. Par composition, on en déduit une famille conservative de points du topos $\widetilde{X_{\text {et }}}(G)$. Notons alors $[X]$ l'ensemble $X$ muni de la topologie discrète et Top $[X]$ le topos associé. La famille de points ci-dessus n'est autre qu'un morphisme de topos $\left(\nu^{*}, \nu_{*}\right): \operatorname{Top}[X] \rightarrow \widetilde{X_{\text {et }}}(G)$ et la flèche d'adjonction $1 \rightarrow \nu_{*} \circ \nu^{*}$ est un monomorphisme, par définition de «conservatif». Voici le résultat crucial de Berkovich :

B.1.6. Proposition. - Pour tout groupe abélien $\mathcal{G}$ sur Top $[X]$, le faisceau $\omega_{X} \iota_{X} \nu_{*}(\mathcal{G})$ sous-jacent à l'objet $\nu_{*}(\mathcal{G})$ de $\operatorname{Mod}_{\mathbb{Z}}\left(\bar{X}_{\mathrm{et}}(G)\right)$ est acyclique pour les foncteurs $\Gamma_{!}$et $\phi_{!}$. 
On en déduit immédiatement

B.1.7. Corollaire. - On a $R \phi_{!}^{\text {disc }} \circ \iota_{X}=\iota_{Y} \circ R \phi_{!}^{\infty}$ et $R \Gamma_{!}^{\text {disc }}(X,-) \circ \iota_{X}=$ $\iota_{\mathrm{pt}} \circ R \Gamma_{!}^{\infty}(X,-)$.

Appliquant le résultat de finitude cohomologique de [2, 5.3.8] on obtient, lorsque $\Lambda$ est $d e$ torsion et $K$ est algébriquement clos, le foncteur

$$
R \Gamma_{!}^{\infty}(X,-): D_{\Lambda}^{b}\left(\widetilde{X_{\mathrm{et}}}(G)\right) \rightarrow D_{\Lambda}^{b}(G)
$$

Nous noterons parfois simplement $R \Gamma_{c}(X,-)$ ou même $R \Gamma_{c}$ ce foncteur.

\section{B.2. Coefficients $l$-adiques}

L'auteur ne connaît pas de formalisme $l$-adique complet pour les espaces analytiques, c'est-àdire l'existence pour chaque espace analytique $X$ d'une catégorie triangulée $D_{c}^{b}\left(X, \overline{\mathbb{Q}}_{l}\right)$ stable par les «six opérations». Berkovich a défini des groupes de cohomologie $l$-adique à supports compacts pour un $\mathbb{Q}_{l}$-faisceau «lisse» (non publié). Il a aussi montré que lorsqu'un tel faisceau est muni d'une action d'un groupe localement pro- $p$ et que l'espace analytique est «quasialgébrique », alors l'action obtenue en cohomologie est lisse, voir [19, 4.1.19].

Néanmoins, ces groupes de cohomologie ne sont pas définis par des foncteurs dérivés. Or, pour le présent article, nous avons évidemment besoin d'un formalisme en catégories dérivées. Ainsi nous allons, en suivant des idées originales de Jannsen [30] reprises par Huber [27] puis Fargues [19, 4.1], attacher à un espace analytique raisonnable $X$ muni d'une action continue d'un groupe $G$ localement pro- $p$-fini, un complexe $R \Gamma_{c}^{\infty}\left(X, \overline{\mathbb{Q}}_{l}\right) \in D^{b}\left(\overline{\mathbb{Q}}_{l} G\right)$ canonique dans la catégorie dérivée des $\overline{\mathbb{Q}}_{l} G$-modules lisses et qui calcule la cohomologie $l$-adique de $X$ munie de l'action de $G$.

Notons que dans le cas qui nous intéresse pour cet article, à savoir l'action de $G L_{d}(K)$ sur $\Omega_{K}^{d-1}$, Harris a construit dans [20] un complexe de représentations lisses ayant la cohomologie souhaitée et l'a notamment utilisé pour définir une suite spectrale d'uniformisation. Sa construction est ad hoc et se généralise mal car elle repose sur l'existence d'un recouvrement par des ouverts distingués quasi-algébriques de nerf l'immeuble de Bruhat-Tits. Quoiqu'il en soit, il résultera des arguments qui suivent que son complexe est un représentant de notre $R \Gamma_{c}^{\infty}$.

\section{B.2.1. La définition de Berkovich}

Fixons un anneau $\Lambda$ de valuation discrète, complet et de caractéristique résiduelle $\neq p$, dont on note $\mathfrak{m}$ l'idéal maximal. Soit $X$ un espace $K$-analytique. Nous conviendrons ici d'appeler un système projectif $\left(\mathcal{F}_{n}\right)_{n}$ de $\Lambda$-faisceaux sur $X$ un « $\Lambda$-système local» si

(i) $\forall n \in \mathbb{N}, \mathcal{F}_{n}$ est un faisceau étale localement constant fini en $\Lambda / \mathfrak{m}^{n}$-modules.

(ii) $\forall m \geqslant n, \mathcal{F}_{m} \otimes \Lambda / \mathfrak{m}^{n} \stackrel{\sim}{\longrightarrow} \mathcal{F}_{n}$.

Notons $\mathbb{U}(X)$ l'ensemble des ouverts distingués de $X$. Berkovich définit pour tout $q \in \mathbb{N}$

$$
H_{c}^{q}\left(X,\left(\mathcal{F}_{n}\right)_{n}\right):=\varliminf_{U \in \mathbb{U}(X)} \varliminf_{n} H_{c}^{q}\left(U, \mathcal{F}_{n}\right) .
$$

Nous poserons aussi

$$
H_{c}^{q}(X, \Lambda):=H_{c}^{q}\left(X,\left(\Lambda / \mathfrak{m}^{n}\right)_{n}\right) \quad \text { et } \quad H_{c}^{q}(X, Q):=Q \otimes H_{c}^{q}(X, \Lambda)
$$

pour toute extension algébrique $Q$ du corps des fractions de $\Lambda$.

$4^{\text {e }}$ SÉRIE - TOME $39-2006-\mathrm{N}^{\circ} 1$ 


\section{B.2.2. La définition de Jannsen-Huber-Fargues}

Commençons par quelques généralités : si $\mathcal{T}$ est un topos, on appelle $\Lambda_{\bullet}$-module de $\mathcal{T}$ tout système projectif $\left(\mathcal{F}_{n}\right)_{n \in \mathbb{N}}$ de $\Lambda$-modules de $\mathcal{T}$ vérifiant $\mathfrak{m}^{n} \mathcal{F}_{n}=0$ pour tout $n \in \mathbb{N}$. Ces objets, munis d'une notion évidente de morphismes, forment une catégorie abélienne $\operatorname{Mod}_{\Lambda} .(\mathcal{T})$ qui, par des arguments généraux sur les systèmes projectifs [30, (1.1)], possède assez d'objets injectifs. Le foncteur bête $\operatorname{Mod}_{\Lambda}(\mathcal{T}) \rightarrow \operatorname{Mod}_{\Lambda} .(\mathcal{T})$ qui envoie un $\Lambda$-module $\mathcal{F}$ sur le système des $\mathcal{F} / \mathfrak{m}^{n} \mathcal{F}$ admet un adjoint à droite

$$
\begin{aligned}
\varliminf^{\mathcal{T}}: \operatorname{Mod}_{\Lambda}(\mathcal{T}) & \rightarrow \operatorname{Mod}_{\Lambda}(\mathcal{T}), \\
\left(\mathcal{F}_{n}\right)_{n \in \mathbb{N}} & \mapsto \varliminf_{n}^{\lim } \mathcal{F}_{n}
\end{aligned}
$$

qui est donc exact à gauche. Si $F$ est un foncteur de source $\operatorname{Mod}_{\Lambda}(\mathcal{T})$, on notera $F_{\bullet}$ le foncteur composé $F_{\bullet}:=F \circ \varliminf^{\mathcal{T}}$ de source $\operatorname{Mod}_{\Lambda}(\mathcal{T})$.

Dans le cas du topos $\widetilde{X_{\text {et }}}$, les objets de $\operatorname{Mod}_{\Lambda}$. $\left(\widetilde{X_{\text {et }}}\right)$ seront simplement appelés « $\Lambda_{\bullet}$-faisceaux étales sur $X »$. Le foncteur «sections à supports compacts»

$$
\Gamma_{!, \bullet}(X,-):=\Gamma_{!}(X,-) \circ \varliminf^{X}: \operatorname{Mod}_{\Lambda}\left(\widetilde{X_{\text {et }}}\right) \rightarrow \operatorname{Mod}_{\Lambda}(\cdot)
$$

est exact à gauche et on peut donc considérer son foncteur dérivé $R \Gamma_{!} \bullet \bullet(X,-)$. Si de plus un groupe discret $G$ agit sur $X$, on note $\Gamma_{!}^{\mathrm{eq}}(X,-): \operatorname{Mod}_{\Lambda}\left(\widetilde{X_{\text {et }}}(G)\right) \rightarrow \operatorname{Mod}_{\Lambda}(G)$ le foncteur des sections à supports compacts et on en déduit un foncteur $\Gamma_{!, \bullet}^{\mathrm{eq}}(X,-)$ (parfois noté simplement $\left.\Gamma_{!, \bullet}^{\mathrm{eq}}\right)$ exact à gauche. D’après L. Fargues, on a alors :

B.2.3. FAIT. - Avec les notations ci-dessus,

(i) $[19,4.1 .4] R \Gamma_{!, \bullet}=R \Gamma_{!} \circ R \varliminf^{X}$, et de même $R \Gamma_{! \bullet \bullet}^{\mathrm{eq}}=R \Gamma_{!}^{\mathrm{eq}} \circ R \varliminf^{X(G)}$, ce qui permet dans chacun des cas de calculer la cohomologie d'un système $\left(\mathcal{F}_{n}\right)_{n}$ comme l'hypercohomologie d'un complexe de $\Lambda$-faisceaux, resp. G-équivariants.

(ii) $\left[19\right.$, lemme 4.2.6] $R \Gamma_{!, \bullet} \circ \omega_{X} \simeq \omega_{\mathrm{pt}} \circ R \Gamma_{! \cdot \bullet}^{\mathrm{eq}}$.

(iii) $[19,4.1 .9] \mathrm{Si}\left(\mathcal{F}_{n}\right)_{n}$ est un $\Lambda$-système local, on a

$$
R^{q} \Gamma_{!, \bullet}\left(X,\left(\mathcal{F}_{n}\right)_{n}\right) \stackrel{\sim}{\longrightarrow} H_{c}^{q}\left(X,\left(\mathcal{F}_{n}\right)_{n}\right),
$$

le terme de droite désignant la cohomologie définie par Berkovich.

Par la troisième propriété ci-dessus, le complexe

$$
R \Gamma_{!, \bullet}^{\mathrm{eq}}\left(X,\left(\Lambda / \mathfrak{m}^{n}\right)_{n}\right) \in D_{\Lambda}^{b}(G)
$$

calcule la cohomologie de $X$ à coefficients dans $\Lambda$ munie de son action de $G$ et possède de bonnes propriétés de fonctorialité (par exemple compatibilité à l'action d'un autre groupe ou de Galois). On veut maintenant adapter la construction précédente au cas où $G$ est topologique et agit continûment sur $X$.

\section{B.2.4. Actions lisses d'un groupe topologique}

On suppose dorénavant que $G$ est localement profini et agit continûment sur $X$. Il y a donc lieu de distinguer les topos $\widetilde{X_{\text {et }}}\left(G_{\text {disc }}\right)$ et $\widetilde{X_{\text {et }}}(G)$ comme au paragraphe B.1.3. Nous remplacerons la notation eq du paragraphe précédent par la notation disc : on a donc un foncteur

$$
\Gamma_{!, \bullet}^{\mathrm{disc}}(X,-): \operatorname{Mod}_{\Lambda}\left(\widetilde{X_{\mathrm{et}}}\left(G_{\mathrm{disc}}\right)\right) \rightarrow \operatorname{Mod}_{\Lambda}\left(G_{\mathrm{disc}}\right) .
$$


Une construction analogue fournit le foncteur exact à gauche

$$
\Gamma_{!, \bullet}^{\infty}(X,-):=\Gamma_{!}^{\infty}(X,-) \circ \varliminf^{X(G)}: \operatorname{Mod}_{\Lambda}\left(\widetilde{X_{\mathrm{et}}}(G)\right) \rightarrow \operatorname{Mod}_{\Lambda}(G)
$$

où l'on rappelle que $\operatorname{Mod}_{\Lambda}(G)$ désigne la catégorie des $\Lambda G$-modules lisses. Il faut prendre garde au fait que le morphisme canonique $\iota_{X} \circ \varliminf^{X(G)} \rightarrow \varliminf^{X\left(G_{\mathrm{disc}}\right)} \circ \iota_{X}$ n'est pas en général un isomorphisme. Par contre il induit un isomorphisme $\varliminf^{X(G)} \stackrel{\sim}{\longrightarrow} \infty_{X} \circ \varliminf^{X\left(G_{\mathrm{disc}}\right)} \circ \iota_{X}$ comme on le vérifie aisément sur les définitions. De même, la transformation naturelle

$$
\Phi: \iota . \circ \Gamma_{!, \bullet}^{\infty} \rightarrow \Gamma_{!, \bullet}^{\text {disc }} \circ \iota_{X}
$$

n'est pas un isomorphisme mais son adjointe

$$
\Psi: \Gamma_{!, \bullet}^{\infty} \rightarrow \infty \circ \Gamma_{!, \bullet}^{\text {disc }} \circ \iota_{X}
$$

est un isomorphisme de foncteurs.

B.2.5. Proposition. - On suppose que G est localement pro-p.

(i) La transformation naturelle $\Psi$ induit pour tout $q \in \mathbb{N}$ des isomorphismes

$$
R^{q} \Psi: R^{q} \Gamma_{!, \bullet}^{\infty} \rightarrow \infty \circ R^{q} \Gamma_{!, \bullet}^{\text {disc }} \circ \iota_{X}
$$

entre foncteurs $\operatorname{Mod}_{\Lambda}\left(\widetilde{X_{\mathrm{et}}}(G)\right) \rightarrow \operatorname{Mod}_{\Lambda}(G)$.

(ii) Supposons $X$ quasi-algébrique. Soit $\left(\mathcal{F}_{n}\right)_{n}$ un $\Lambda$-système local G-équivariant. Alors la transformation naturelle $\Phi$ induit un isomorphisme

$$
R \Phi\left(\left(\mathcal{F}_{n}\right)_{n}\right): \iota_{\mathrm{pt}} R \Gamma_{!, \bullet}^{\infty}\left(X,\left(\mathcal{F}_{n}\right)_{n}\right) \rightarrow R \Gamma_{!, \bullet}^{\text {disc }}\left(X,\left(\iota_{X} \mathcal{F}_{n}\right)_{n}\right) .
$$

En particulier, on a des isomorphismes canoniques de $\Lambda G$-modules (lisses)

$$
R^{q} \Gamma_{!, \bullet}^{\infty}\left(X,\left(\mathcal{F}_{n}\right)_{n}\right) \stackrel{\sim}{\longrightarrow} H_{c}^{q}\left(X,\left(\mathcal{F}_{n}\right)_{n}\right) .
$$

(iii) Sous les hypothèses précédentes on a pour $m \in \mathbb{N}^{\times}$des isomorphismes canoniques

$$
R \Gamma_{!, \bullet}^{\infty}\left(X,\left(\mathcal{F}_{n}\right)_{n}\right) \otimes_{\Lambda}^{L} \Lambda / \mathfrak{m}^{m} \stackrel{\sim}{\longrightarrow} R \Gamma_{!}^{\infty}\left(X, \mathcal{F}_{m}\right) .
$$

(iv) On a $R \Gamma_{!, \bullet}^{\infty}=R \Gamma_{!}^{\infty} \circ R \varliminf^{X(G)}$.

Avant de prouver cette proposition, introduisons les notations utilisées dans la section 4 :

B.2.6. Notation. - Soit $\Lambda$ un anneau commutatif unitaire tel que $p \in \Lambda^{\times}$. Si $\Lambda$ est de torsion, on notera

$$
R \Gamma_{c}(X, \Lambda):=R \Gamma_{!}^{\infty}(X, \Lambda) \in D_{\Lambda}^{b}(G) .
$$

Si $\Lambda$ est complet de valuation discrète, $Q$ est une extension algébrique de $\operatorname{Frac}(\Lambda)$, et sous les hypothèse supplémentaires que $X$ est quasi-algébrique et $G$ localement pro- $p$, on pose

$$
R \Gamma_{c}(X, \Lambda):=R \Gamma_{!, \bullet}^{\infty}\left(X,\left(\Lambda / \mathfrak{m}^{n}\right)_{n}\right) \quad \text { et } \quad R \Gamma_{c}(X, Q):=Q \otimes_{\Lambda} R \Gamma_{c}(X, \Lambda) .
$$

Le reste de cette section est consacré à la preuve de la proposition B.2.5. Nous aurons besoin du lemme technique suivant :

$4^{\mathrm{e}}$ SÉRIE - TOME $39-2006-\mathrm{N}^{\circ} 1$ 


\section{B.2.7. LeMme. - On a $R\left(\Gamma_{!, \bullet}^{\text {disc }} \circ \iota_{X}\right)=R \Gamma_{!, \bullet}^{\text {disc }} \circ \iota_{X}$}

que nous admettrons momentanément.

\section{B.2.8. Preuve de B.2.5(i)}

Admettant le lemme précédent, le problème à régler est le suivant : le foncteur de lissification $\infty$ n'est pas exact sur la catégorie des $\Lambda G_{\text {disc }}$-modules et « dériver » l'isomorphisme $\Psi: \Gamma_{! \cdot \bullet}^{\infty} \sim{ }^{\longrightarrow} \infty \circ \Gamma_{!, \bullet}^{\text {disc }} \circ \iota_{X}$ devrait donc faire intervenir le foncteur $R \infty$. Ce qu'affirme le point (i) qu'on veut prouver, c'est qu'il n'en est rien. La preuve est technique mais l'idée est simple : on va voir que le foncteur $\Gamma_{! \cdot \bullet}^{\text {disc }} \circ \iota_{X}$ envoie la catégorie $\operatorname{Mod}_{\Lambda} .\left(\widetilde{X_{\text {et }}}(G)\right)$ dans une sous-catégorie $\infty$-acyclique de $\operatorname{Mod}_{\Lambda}\left(G_{\text {disc }}\right)$.

Pour formaliser tout ça, nous devons introduire auparavant une certaine catégorie de $\Lambda G$-modules, intermédiaire entre la catégorie lisse et la catégorie de tous les $\Lambda G$-modules.

Modules sur l'algèbre de distributions : Soient $G$ un groupe localement pro- $p$ et $\Lambda$ un anneau tel que $p \in \Lambda^{\times}$. Notons $\mathcal{D}_{\Lambda}(G)$, resp. $\mathcal{H}_{\Lambda}(G)$, l'algèbre de convolution des mesures, resp. mesures localement constantes, à support compact et à valeurs dans $\Lambda$ sur $G$. L'anneau $\mathcal{H}_{\Lambda}(G)$ n'a pas d'unité mais assez d'idempotents et on sait que la catégorie des $\Lambda G$-modules lisses est canoniquement isomorphe à la catégorie des $\mathcal{H}_{\Lambda}(G)$-modules lisses (appelés aussi nondégénérés ou unitaux). L'anneau $\mathcal{D}_{\Lambda}(G)$ est unitaire et contient les anneaux $\Lambda[G]$ et $\mathcal{H}_{\Lambda}(G)$. On a $\mathcal{D}_{\Lambda}(G) * \mathcal{H}_{\Lambda}(G)=\mathcal{H}_{\Lambda}(G)$ de sorte que toute structure de $\mathcal{H}_{\Lambda}(G)$-module lisse s'étend canoniquement en une structure de $\mathcal{D}_{\Lambda}(G)$-module : en effet, si $M$ est un $\mathcal{H}_{\Lambda}(G)$-module lisse, $m \in M$ et $d \in \mathcal{D}_{\Lambda}(G)$, on pose $d . m:=(d * e) . m$, expression qui ne dépend pas du choix de l'idempotent $e$ de $\mathcal{H}_{\Lambda}(G)$ fixant $m$. On obtient le foncteur $\iota_{\infty}^{D}$ du système de foncteurs suivant :

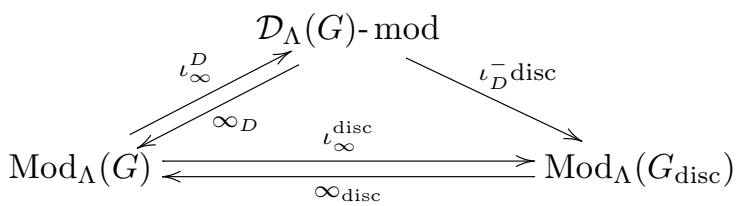

Les autres foncteurs $\iota$ sont des foncteurs d'oublis. Les foncteurs $\infty$ sont des foncteurs de lissification ; celui qui est noté $\infty_{\text {disc }}$ est le foncteur de lissification «habituel » que nous notons ailleurs simplement $\infty$ et qui à un $\Lambda G_{\text {disc }}$-module associe le sous-module de ses vecteurs lisses. On définit $\infty_{D}$ pour tout $\mathcal{D}_{\Lambda}(G)$-module $M$ par la formule

$$
\infty_{D}(M):=\underset{H \subset G}{\lim _{H \subset G}} e_{H} M,
$$

la limite étant prise sur un système de voisinages de l'unité formé de pro-p-sous-groupes ouverts, et la notation $e_{H}$ désignant l'idempotent de $\mathcal{H}_{\Lambda}(G)$ associé à $H$. L'avantage d'avoir introduit la catégorie $\mathcal{D}_{\Lambda}(G)-\bmod$ est que le foncteur $\infty_{D}$ y est exact. Cependant, on prendra garde au fait qu'en général, $\infty_{D}(M) \neq \infty_{\text {disc }}\left(\iota_{D}^{\text {disc }}(M)\right)$. Néanmoins cet inconvénient sera inoffensif pour l'utilisation que nous ferons de ces objets. La motivation pour introduire l'anneau $\mathcal{D}_{\Lambda}(G)$ est la remarque suivante : soit $\left(M_{n}\right)_{n}$ un système projectif de $\Lambda G$-modules lisses. Le $\Lambda G_{\text {disc }^{-}}$ module «limite projective» $\lim _{n} M_{n}$ n'est en général pas lisse, mais est canoniquement muni d'une structure de $\mathcal{D}_{\Lambda}(G)$-module. De plus on a $\infty_{D}\left(\varliminf_{n} M_{n}\right)=\infty_{\text {disc }}\left(\iota_{D}^{\operatorname{disc}}\left(\varliminf_{n} M_{n}\right)\right)$.

On obtient de cette manière un foncteur de la catégorie des systèmes projectifs de $\Lambda G$-modules lisses vers celle des $\mathcal{D}_{\Lambda}(G)$-modules. En particulier, si $\left(\mathcal{F}_{n}\right)_{n}$ est un $\Lambda_{\bullet}$-faisceau $G$-équivariant, on a une structure de $\mathcal{D}_{\Lambda}(G)$-module sur $\varliminf_{l} \Gamma_{!}^{\text {disc }}\left(X, \iota_{X} \mathcal{F}_{n}\right)$. Or, puisque les foncteurs de sections $\Gamma(U,-)$ commutent aux limites projectives dans $\widetilde{X_{\mathrm{et}}}\left(G_{\mathrm{disc}}\right)$ (comme dans $\widetilde{X_{\mathrm{et}}}$ ), on 
a une inclusion

$$
\Gamma_{!, \bullet}^{\text {disc }}\left(X,\left(\iota_{X} \mathcal{F}_{n}\right)_{n}\right)=\Gamma_{!}^{\text {disc }}\left(X, \varliminf^{\widetilde{X_{\text {et }}}\left(G_{\text {disc }}\right)} \iota_{X} \mathcal{F}_{n}\right) \subseteq \varliminf_{\lim }^{\text {disc }}\left(X, \iota_{X} \mathcal{F}_{n}\right) .
$$

Puisque les mesures de $\mathcal{D}_{\Lambda}(G)$ sont à supports compacts, le sous- $\Lambda G$-module $\Gamma_{!}^{\text {disc }}\left(X, \varliminf^{\widetilde{X_{\text {et }}}\left(G_{\text {disc }}\right)} \iota_{X} \mathcal{F}_{n}\right)$ est stable par $\mathcal{D}_{\Lambda}(G)$, de sorte qu'on obtient un foncteur

$$
\begin{aligned}
\Gamma_{!, \bullet}^{D}: \operatorname{Mod}_{\Lambda}\left(\widetilde{X_{\text {et }}}(G)\right) & \rightarrow \mathcal{D}_{\Lambda}(G)-\bmod , \\
\left(\mathcal{F}_{n}\right)_{n} & \mapsto \Gamma_{!}^{\operatorname{disc}}\left(X, \varliminf_{n}^{\lim } \iota_{X} \mathcal{F}_{n}\right)
\end{aligned}
$$

qui «factorise» $\Psi$ en deux isomorphismes de foncteurs :

$$
\Psi^{D}: \Gamma_{!, \bullet}^{\infty} \stackrel{\sim}{\longrightarrow} \infty_{D} \circ \Gamma_{!, \bullet}^{D} \quad \text { et } \quad \Phi_{D}: \iota_{D}^{\text {disc }} \circ \Gamma_{!, \bullet}^{D} \stackrel{\sim}{\longrightarrow} \Gamma_{!, \bullet}^{\text {disc }} \circ \iota_{X}
$$

que l'on peut dériver en

$$
R \Psi^{D}: R \Gamma_{!, \bullet}^{\infty} \stackrel{\sim}{\longrightarrow} \infty_{D} \circ R \Gamma_{!, \bullet}^{D} \quad \text { et } \quad R \Phi_{D}: \iota_{D}^{\text {disc }} \circ R \Gamma_{!, \bullet}^{D} \stackrel{\sim}{\longrightarrow} R \Gamma_{!, \bullet}^{\text {disc }} \circ \iota_{X}
$$

en utilisant l'exactitude de $\infty_{D}$ pour la première et le lemme B.2.7 pour la deuxième. Soit maintenant $q \in \mathbb{N}$; en combinant les divers isomorphismes ci-dessus on obtient

$$
R^{q} \Gamma_{!, \bullet}^{\infty} \stackrel{\sim}{\longrightarrow} \infty_{D} \circ R^{q} \Gamma_{!, \bullet}^{D} \simeq \infty_{\text {disc }} \circ \iota_{D}^{\text {disc }} \circ R^{q} \Gamma_{!, \bullet}^{D} \stackrel{\sim}{\longrightarrow} \infty \circ R^{q} \Gamma_{!, \bullet}^{\text {disc }} \circ \iota_{X} .
$$

\section{B.2.9. Preuve de B.2.5(ii) et (iii)}

Remarquons tout d'abord que d'après le point (i), $R^{q} \Gamma_{c}^{\infty}\left(X,\left(\mathcal{F}_{n}\right)_{n}\right)$ est différent de $R^{q} \Gamma_{c}\left(X,\left(\mathcal{F}_{n}\right)_{n}\right)$ dès que ce dernier n'est pas lisse pour l'action de $G$, ce qui est par exemple le cas pour le système $\left(j !\left(\mathbb{Z} / l^{n}\right)\right)_{n}$ où $j$ est l'inclusion de $\Omega_{K}^{d-1}$ dans $\mathbb{P}_{K}^{d-1}$.

Cependant on sait par Berkovich (voir aussi $[19,4.1 .19]$ ) que sous l'hypothèse technique de quasi-algébricité de $X$, cf. [19, 4.1.11], et parce que nous avons supposé $G$ localement pro- $p$ et $p \in \Lambda^{\times}$, l'action de $G$ sur les groupes de cohomologie à supports compacts d'un $\Lambda$-système local $G$-équivariant $\left(\mathcal{F}_{n}\right)_{n}$ est lisse. On en déduit le point (ii). À partir de là, il suffit pour prouver le point (iii) d'y remplacer $R \Gamma_{!, \bullet}^{\infty}$ par $R \Gamma_{!, \bullet}$ et $R \Gamma_{!}^{\infty}$ par $R \Gamma_{\text {!. }}$ Dans ce cas on trouve les arguments dans la preuve de [19, 4.1.17].

\section{B.2.10. Preuve du lemme B.2.7 et de B.2.5(iv)}

Commençons par marier les techniques de Berkovich à celles de Jannsen. On utilise le morphisme de topos $\left(\nu^{*}, \nu_{*}\right): \operatorname{Top}[X] \rightarrow \widetilde{X_{\text {et }}}(G)$ de la proposition B.1.6. Soit $\left(\mathcal{F}_{n}\right)_{n}$ un objet de $\operatorname{Mod}_{\Lambda}\left(\widetilde{X_{\text {et }}}(G)\right)$. D'après [30, (1.1)], on peut choisir un plongement de $\nu^{*}\left(\mathcal{F}_{n}\right)_{n}$ dans un objet injectif $\left(\mathcal{I}_{n}\right)_{n}$ de $\operatorname{Mod}_{\Lambda}$. $(\operatorname{Top}[X])$. Puisque $[X]$ est un ensemble conservatif de points, on a par adjonction un plongement de $\left(\mathcal{F}_{n}\right)_{n}$ dans $\nu_{*}\left(\mathcal{I}_{n}\right)_{n}$ qui est un objet injectif $\operatorname{de} \operatorname{Mod}_{\Lambda}$. $\left(\widetilde{X_{\text {et }}}(G)\right)$. Or, on a

$$
\varliminf^{X(G)}\left(\nu_{*}\left(\mathcal{I}_{n}\right)_{n}\right) \simeq \nu_{*}\left(\varliminf^{\operatorname{Top}[X]} \mathcal{I}_{n}\right)
$$

par commutation des images directes et des produits. Ainsi, d'après la proposition B.1.6, le $\Lambda$-faisceau $\varliminf^{X(G)}\left(\nu_{*}\left(\mathcal{I}_{n}\right)_{n}\right)$ est $\Gamma_{\text {! }}$-acyclique. On a donc vérifié que le foncteur $\varliminf^{X(G)}$ envoie assez d'objets injectifs sur des objets $\Gamma_{\text {! }}$-acycliques, et on en déduit le point (iv) de B.2.5.

Passons à la preuve du lemme B.2.7. Il s'agit de voir que $\iota_{X}$ envoie suffisamment d'objets de la catégorie $\operatorname{Mod}_{\Lambda_{\bullet}}\left(\widetilde{X_{\text {et }}}(G)\right)$ sur des objets $\Gamma_{!, \bullet}^{\text {disc }}$-acycliques de $\operatorname{Mod}_{\Lambda} \cdot\left(\widetilde{X_{\text {et }}}\left(G_{\text {disc }}\right)\right)$. Soit 
$\mathbb{U}(X)$ l'ensemble des ouverts distingués de $X$. D'après [19, Prop. 4.1.8], on a pour tout objet $\left(\mathcal{G}_{n}\right)_{n}$ de $\operatorname{Mod}_{\Lambda}\left(\widetilde{X_{\text {et }}}(G)\right)$ et tout $p>0$ une suite exacte de $\Lambda$-modules

$$
\begin{aligned}
& 0 \rightarrow \varliminf_{U \in \mathbb{U}(X)} R^{1} \underbrace{\varliminf_{i m}}_{n} R^{p-1} \Gamma_{!}\left(U, \omega_{X} \iota_{X} \mathcal{G}_{n}\right) \rightarrow \omega_{\mathrm{pt}} R^{p} \Gamma_{!, \bullet}^{\text {disc }}\left(X,\left(\iota_{X} \mathcal{G}_{n}\right)_{n}\right) \\
& \rightarrow \varliminf_{U \in \mathbb{U}(X)}^{\varliminf_{n}} \underbrace{\lim _{n}}_{n} \Gamma_{!}\left(U, \omega_{X} \iota_{X} \mathcal{G}_{n}\right) \rightarrow 0
\end{aligned}
$$

de laquelle on tire que si pour tout $U \in \mathbb{U}(X)$, les faisceaux $\omega_{X} \iota_{X} \mathcal{G}_{n}$ sont tous $\Gamma_{!}(U,-)$ acycliques et le système projectif de $\Lambda$-modules $\left(\Gamma_{!}\left(U, \omega_{X} \iota_{X} \mathcal{G}_{n}\right)\right)_{n}$ satisfait la condition de Mittag-Leffler, alors le système $\left(\iota_{X} \mathcal{G}_{n}\right)_{n}$ est $\Gamma_{!, \bullet}^{\text {disc }}$-acyclique. Or pour un système $\left(\mathcal{I}_{n}\right)_{n}$ construit comme dans [30, (1.1)], les applications de transitions $\Gamma_{!}\left(U, \omega_{X} \iota_{X} \mathcal{I}_{n}\right) \rightarrow$ $\Gamma_{!}\left(U, \omega_{X} \iota_{X} \mathcal{I}_{m}\right)$ sont même surjectives. Un tel système $\left(\mathcal{I}_{n}\right)_{n}$ est donc $\Gamma_{!, \bullet}^{\text {disc }}$-acyclique.

\section{B.3. Suites spectrales de Hochschild-Serre}

Dans cette section, on s'intéresse à la situation suivante : un groupe discret $G$ agit librement et proprement sur un $K$-espace analytique $X$. On sait alors former le quotient $X / G$, cf. [6, lemma 4], et l'application quotient $X \stackrel{p}{\rightarrow} X / G$ est un revêtement analytique galoisien de groupe $G$. En particulier, $p$ est donc étale. Dans ce contexte, $[19,4.4]$ montre que pour un «faisceau $l$-adique » ou sur un anneau Gorenstein fini, il existe une suite spectrale de HochschildSerre, c'est-à-dire du type :

$$
E_{2}^{p q}=\operatorname{Ext}_{G}^{p}\left(H_{c}^{q}\left(X, p^{*} \mathcal{F}\right), 1\right) \Longrightarrow H_{c}^{-q-p}(X / G, \mathcal{F})^{*} .
$$

Nous avons besoin d'une interprétation en termes de catégories dérivées de cette suite spectrale. Ceci nous conduira d'ailleurs à donner une preuve différente de celle de [19, 4.4]. Le résultat principal de cette section s'énonce ainsi (les notations seront expliquées au fil de la preuve) :

B.3.1. Proposition. - Soient $G$ un groupe discret agissant librement et proprement sur un $K$-espace analytique $X$ et $p: X \rightarrow X / G$ le quotient. On suppose $K$ algébriquement clos. Si $\Lambda$ est un anneau de torsion première à $p$ ou un anneau l-adique, alors il existe un isomorphisme de foncteurs $D_{\Lambda}^{b}\left(\widetilde{X / G_{\mathrm{et}}}\right) \rightarrow D^{-}(\Lambda)$, resp. $D_{\Lambda}^{b}\left(\widetilde{X / G_{\mathrm{et}}}\right) \rightarrow D^{-}(\Lambda)$

$$
\Phi: \Lambda \otimes_{\Lambda[G]}^{L}\left(R \Gamma_{c}^{\mathrm{eq}}\left(X, p^{*}(\cdot)\right)\right) \stackrel{\sim}{\longrightarrow} R \Gamma_{c}(X / G, \cdot),
$$

où $\Gamma_{c}$ désigne le foncteur $\Gamma_{!}$, resp. $\Gamma_{!}, \bullet$ des sections précédentes.

L'hypothèse sur $K$ n'est pas incontournable : il suffirait de supposer que la dimension cohomologique des $\operatorname{Gal}\left(K^{\mathrm{ca}} / K\right)$-modules de $l$-torsions est bornée pour obtenir les résultats de cette section sur un anneau de $l$-torsion ou $l$-adique. Quoiqu'il en soit, avec cette hypothèse on sait que :

- $H_{c}^{q}(X, \mathcal{F})=0$ pour $q>2 \operatorname{dim}(X)$ et tout faisceau abélien de torsion, [2, 5.3.8].

- $H_{c}^{q}\left(X,\left(\mathcal{F}_{n}\right)\right)=0$ pour $q>2 \operatorname{dim}(X)+1$ pour tout $\Lambda_{\bullet}$-faisceau étale comme en B.2.2, si $\Lambda$ est un anneau $l$-adique, $l \neq p,[19,4.1 .9(b)]$.

\section{B.3.2. Quelques sorites}

Remarquons tout d'abord que comme l'action de $G$ sur $X / G$ est triviale, la catégorie $\operatorname{Mod}_{\Lambda}\left(\widehat{X / G_{\text {et }}}(G)\right)$ des $\Lambda$-faisceaux $G$-équivariants sur $X / G$ est équivalente à la catégorie $\operatorname{Mod}_{\Lambda[G]}\left(\widehat{X / G_{\text {et }}}\right)$ des faisceaux étales en $\Lambda[G]$-modules sur $X / G$. Ainsi le foncteur 
$\theta: \operatorname{Mod}_{\Lambda}(\cdot) \rightarrow \operatorname{Mod}_{\Lambda}(G)$ qui munit un $\Lambda$-module de l'action triviale de $G$ induit aussi un foncteur $\theta: \operatorname{Mod}_{\Lambda}\left(\widetilde{X / G_{\mathrm{et}}}\right) \rightarrow \operatorname{Mod}_{\Lambda}\left(\widetilde{X / G_{\mathrm{et}}}(G)\right)$. Ces foncteurs ont pour adjoints à gauche les foncteurs $M \mapsto \Lambda \otimes_{\Lambda[G]} M$, resp. $\mathcal{F} \mapsto \Lambda \otimes_{\Lambda[G]} \mathcal{F}$ où le morphisme $\Lambda[G] \rightarrow \Lambda$ est l'augmentation habituelle. La même discussion s'applique verbatim aux catégories de $\Lambda_{\bullet}$-modules.

Notation : pour éviter les confusions nous utiliserons les notations $\Gamma_{c}$, resp. $\Gamma_{c}^{\mathrm{eq}}$, pour désigner le foncteur des sections à support compact lorsqu'on le voit à valeurs dans les $\Lambda$-modules, resp. dans les $\Lambda G$-modules. Par exemple, on a $\Gamma_{c}^{\mathrm{eq}}(X / G, \theta(-))=\theta \circ \Gamma_{c}(X / G,-)$ Les mêmes notations s'appliquent aux $\Lambda_{\bullet}$-faisceaux et aux foncteurs $\Gamma_{c}:=\Gamma_{!, \bullet}=\Gamma_{!} \circ \varliminf_{\text {lim. }}$.

\section{B.3.3. Le morphisme $\Phi$}

Comme $p$ est un morphisme étale, le foncteur $p^{*}: \widetilde{X / G_{\text {et }}}(G) \rightarrow \widetilde{X_{\text {et }}}(G)$ a un adjoint à gauche exact $p_{!}$, voir [2, 5.4.2(ii)]. Le morphisme d'adjonction $p_{!} p^{*} \rightarrow$ Id induit donc un morphisme de foncteurs $\operatorname{Mod}_{\Lambda}\left(\widetilde{X / G_{\text {et }}}\right) \rightarrow \operatorname{Mod}_{\Lambda}(G)$

$$
\Gamma_{c}^{\mathrm{eq}}\left(X, p^{*} \theta(-)\right) \rightarrow \theta\left(\Gamma_{c}(X / G,-)\right) .
$$

Lorsque $\Lambda$ est de torsion, par les résultats de finitude cohomologique rappelés plus haut, on peut dériver ceci en un morphisme de foncteurs $D_{\Lambda}^{b}\left(\widetilde{X / G_{\text {et }}}\right) \rightarrow D_{\Lambda}^{b}(G)$

$$
R \Gamma_{c}^{\mathrm{eq}}\left(X, p^{*} \theta(\cdot)\right) \rightarrow \theta\left(R \Gamma_{c}(X / G, \cdot)\right)
$$

qui par adjonction fournit un morphisme canonique de foncteurs $D_{\Lambda}^{b}\left(\widetilde{X / G_{\text {et }}}\right) \rightarrow D^{-}(\Lambda)$

$$
\Phi: \Lambda \otimes_{\Lambda[G]}^{L}\left(R \Gamma_{c}^{\mathrm{eq}}\left(X, p^{*} \theta(\cdot)\right)\right) \rightarrow R \Gamma_{c}(X / G, \cdot) .
$$

Lorsque $\Lambda$ est $l$-adique, on construit $\Phi$ de la manière suivante : on vérifie d'abord (voir preuve de [19, 4.2.7]) que pour tout morphisme étale $U \stackrel{f}{\rightarrow} V$ et tout $\Lambda_{\bullet}$-faisceau sur $V$, l'isomorphisme évident $f^{*} \lim \left(\mathcal{F}_{n}\right) \stackrel{\sim}{\longrightarrow} \lim \left(f^{*}\left(\mathcal{F}_{n}\right)\right)$ se dérive en un isomorphisme $f^{*} R \varliminf\left(\mathcal{F}_{n}\right) \stackrel{\sim}{\longrightarrow}$ $R \nsupseteq\left(f^{*}\left(\mathcal{F}_{n}\right)\right)$. On en déduit alors un morphisme fonctoriel en $\left(\mathcal{F}_{n}\right)_{n}$

$$
\begin{aligned}
R \Gamma_{c}\left(V,\left(f^{*} \mathcal{F}_{n}\right)_{n}\right) & \simeq R \Gamma_{!}\left(V, R \varliminf\left(f^{*} \mathcal{F}_{n}\right)\right) \\
& \simeq R \Gamma_{!}\left(V, f^{*} R \varliminf_{(i m}\left(\mathcal{F}_{n}\right)\right) \rightarrow R \Gamma_{!}\left(U, R \varliminf\left(\mathcal{F}_{n}\right)\right)=R \Gamma_{c}\left(U,\left(\mathcal{F}_{n}\right)_{n}\right),
\end{aligned}
$$

la flèche du milieu étant encore induite par l'adjonction $f_{!} f^{*} \rightarrow$ Id.

Appliquant ceci au morphisme $p$, et compte tenu de la finitude cohomologique rappelée plus haut, on obtient formellement de la même manière que pour les faisceaux de torsion un morphisme de foncteurs $D_{\Lambda \cdot}^{b}\left(\widetilde{X / G_{\text {et }}}\right) \rightarrow D^{-}(\Lambda)$ :

$$
\Phi: \Lambda \otimes_{\Lambda[G]}^{L}\left(R \Gamma_{c}^{\mathrm{eq}}\left(X, p^{*} \theta(\cdot)\right)\right) \rightarrow R \Gamma_{c}(X / G, \cdot) .
$$

B.3.4. Proposition. - Dans les deux cas considérés, $\Phi$ est un isomorphisme de foncteurs.

Preuve. - Nous dirons qu'un faisceau, resp. un $\Lambda_{\bullet}$-faisceau, $\mathcal{F}$ sur $X / G_{\text {et }}$ est :

- $\Gamma_{c}$-acyclique s'il est $\Gamma_{c}(U,-)$-acyclique pour tout morphisme étale $U \rightarrow X / G$.

- $\Gamma_{c, \text { et }}^{\vee}$-acyclique, resp. $\Gamma_{c, \text { loc }}^{\vee}$-acyclique si pour tout morphisme surjectif $U \rightarrow V$ étale, resp. localement iso, entre ouverts étales au-dessus de $X / G$, le complexe de Cech «dual»

$$
\cdots \Longrightarrow \Gamma_{c}\left(U \times_{V} U, \mathcal{F}\right) \Longrightarrow \Gamma_{c}(U, \mathcal{F}) \longrightarrow \Gamma_{c}(V, \mathcal{F}) \longrightarrow 0
$$

est acyclique.

$4^{\text {e }}$ SÉRIE - TOME $39-2006-\mathrm{N}^{\circ} 1$ 
Un morphisme $U \stackrel{f}{\rightarrow} V$ est dit localement-iso si tout point de $U$ admet un voisinage ouvert $\mathcal{U}$ tel que $f_{\mid \mathcal{U}}: \mathcal{U} \rightarrow f(\mathcal{U})$ est un isomorphisme. Un tel morphisme est en particulier étale.

B.3.5. LEMmE. - Tout faisceau de torsion, resp. $\Lambda_{\bullet}$-faisceau, sur $X / G_{\mathrm{et}}$ admet une résolution bornée par des faisceaux $\Gamma_{c}$-acycliques et $\Gamma_{c, \text { et }}^{\vee}$-acycliques, resp. par des $\Lambda_{\bullet}$-faisceaux $\Gamma_{c}$-acycliques et $\Gamma_{c, \text { loc }}^{\vee}$-acycliques.

Laissons ce lemme de côté et commençons la preuve de la proposition. Fixons un objet $\mathcal{F}$ de $\operatorname{Mod}_{\Lambda}\left(\widetilde{X / G_{\text {et }}}\right)$ ou de $\operatorname{Mod}_{\Lambda}\left(\widetilde{X / G_{\text {et }}}\right)$, et soit $\mathcal{F} \hookrightarrow \mathcal{J} \bullet$ une résolution bornée de $\mathcal{F}$ comme dans le lemme ci-dessus. Par définition on a :

$$
R \Gamma_{c}(X / G, \mathcal{F})=\Gamma_{c}\left(X / G, \mathcal{J}^{\bullet}\right) \quad \text { et } \quad R \Gamma_{c}^{\mathrm{eq}}\left(X, p^{*} \theta \mathcal{F}\right)=\Gamma_{c}^{\mathrm{eq}}\left(X, p^{*} \theta \mathcal{J}^{\bullet}\right)
$$

(en remarquant que $p^{*} \theta\left(\mathcal{J}^{\bullet}\right)$ est une résolution de $p^{*} \theta(\mathcal{F})$ par des $G$-faisceaux $\Gamma_{c}^{\mathrm{eq}}(X, \cdot)$ acycliques). La proposition est alors une conséquence immédiate du lemme suivant :

B.3.6. Lemme. - Soit $\mathcal{J}$ un faisceau de torsion, resp. $\Lambda_{\bullet}$-faisceau, $\Gamma_{c, \text { loc }}^{\vee}$-acyclique sur $X / G_{\text {et }}$. Alors

(i) $\Gamma_{c}^{\mathrm{eq}}\left(X, p^{*} \theta \mathcal{J}\right)$ est un $\Lambda[G]$-module acyclique pour le foncteur $\Lambda \otimes_{\Lambda[G]}-$.

(ii) Le morphisme canonique $\Lambda \otimes_{\Lambda[G]} \Gamma_{c}^{\mathrm{eq}}\left(X, p^{*} \theta \mathcal{J}\right) \rightarrow \Gamma_{c}(X / G, \mathcal{J})$ est un isomorphisme.

Prouvons ce lemme. Soit $U \stackrel{f}{\rightarrow} X / G$ un morphisme étale. Considérons le produit cartésien $U \times_{X / G} X \stackrel{\pi_{X}}{\longrightarrow} X$ comme un ouvert étale $G$-équivariant sur $X$ en le munissant de l'action Id $\times$ act $_{X}$. Le $\Lambda$-module $\Gamma_{c}\left(U \times_{X / G} X, p^{*} \theta \mathcal{F}\right)$ est alors naturellement muni d'une action de $G$ et nous noterons $\Gamma_{c}^{\mathrm{eq}}\left(U \times_{X / G} X, p^{*} \theta \mathcal{F}\right)$ pour préciser que l'on tient compte de cette action. La première projection fournit un morphisme équivariant $\Gamma_{c}^{\mathrm{eq}}\left(U \times_{X / G} X, p^{*} \theta \mathcal{F}\right) \rightarrow \theta\left(\Gamma_{c}(U, \mathcal{F})\right)$. Dans le cas $U=X / G$, ce morphisme est celui qui induit notre morphisme $\Phi$.

Supposons maintenant que le morphisme $f$ se factorise $U \stackrel{f^{\prime}}{\longrightarrow} X \stackrel{p}{\longrightarrow} X / G$. Alors le morphisme d'espaces analytiques au-dessus de $U$

$$
\begin{aligned}
U \times G & \rightarrow U \times_{X / G} X, \\
(u, g) & \mapsto\left(u, g \cdot f^{\prime}(u)\right)
\end{aligned}
$$

est un isomorphisme $G$-équivariant si l'on munit $U \times G$ de l'action par translation à gauche sur le second terme, qui s'inscrit dans le diagramme :

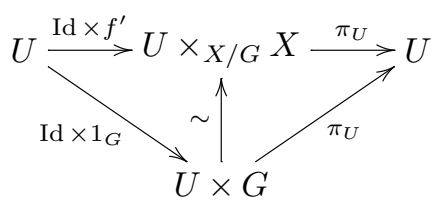

On en déduit, dans le cas de torsion comme dans le cas des $\Lambda_{\bullet}$-faisceaux, un isomorphisme de $\Lambda[G]$-modules fonctoriel en $\mathcal{F}$ :

$$
\Lambda[G] \otimes_{\Lambda} \Gamma_{c}(U, \mathcal{F}) \stackrel{\sim}{\longrightarrow} \Gamma_{c}^{\mathrm{eq}}\left(U \times_{X / G} X, p^{*} \theta \mathcal{F}\right)
$$


qui s'inscrit dans un diagramme

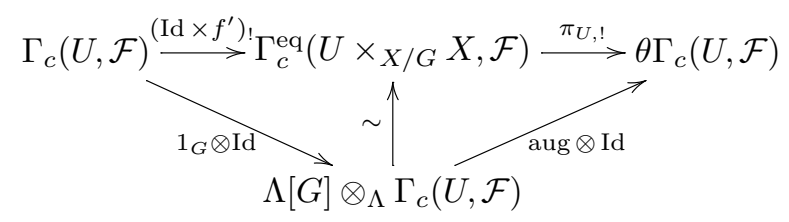

où le triangle de droite est $G$-équivariant mais pas celui de gauche en général. Remarquons que l'isomorphisme ci-dessus dépend de la section $f^{\prime}$ de $f$ contrairement à l'isomorphisme obtenu en composant avec le foncteur $\Lambda \otimes_{\Lambda[G]}-$

$$
\Gamma_{c}(U, \mathcal{F}) \stackrel{\sim}{\longrightarrow} \Lambda \otimes_{\Lambda[G]} \Gamma_{c}^{\mathrm{eq}}\left(U \times_{X / G} X, p^{*} \theta \mathcal{F}\right)
$$

qui n'est autre que l'inverse du morphisme adjoint au morphisme noté $\pi_{U, !}$ dans le diagramme.

Notons maintenant $X^{(0)}:=X / G$ et pour tout $i>0$ définissons par récurrence

$$
X^{(i)}:=X^{(i-1)} \times_{X / G} X \quad \text { et } \quad p^{(i)}: X^{(i)} \rightarrow X / G \text { la projection canonique. }
$$

Appliquant la discussion précédente au morphisme étale $f=p^{(i)}$, on obtient pour $i \geqslant 1$ des isomorphismes

$$
\Lambda[G] \otimes_{\Lambda} \Gamma_{c}\left(X^{(i)}, \mathcal{F}\right) \stackrel{\sim}{\longrightarrow} \Gamma_{c}^{\mathrm{eq}}\left(X^{(i+1)}, p^{*} \theta \mathcal{F}\right)
$$

(dépendant du choix d'une projection $X^{(i)} \rightarrow X$ ) et des isomorphismes

$$
\Lambda \otimes_{\Lambda[G]} \Gamma_{c}^{\mathrm{eq}}\left(X^{(i+1)}, p^{*}(\theta \mathcal{F})\right) \stackrel{\sim}{\longrightarrow} \Gamma_{c}\left(X^{(i)}, \mathcal{F}\right)
$$

canoniques, donc en particulier compatibles aux différentes projections $X^{(i)} \rightarrow X^{(i-1)}$.

Par ailleurs, on peut écrire le complexe de Cech «dual»d'un faisceau $\mathcal{F}$ sur $X / G_{\text {et }}$ associé à un recouvrement $X \times_{X / G} U \stackrel{\pi_{U}}{\longrightarrow} U$ sous la forme

$$
\begin{aligned}
\cdots & \rightarrow \Gamma_{c}\left(X^{(i)} \times_{X / G} U, \mathcal{F}\right) \rightarrow \Gamma_{c}\left(X^{(i-1)} \times_{X / G} U, \mathcal{F}\right) \rightarrow \cdots \\
& \rightarrow \Gamma_{c}\left(X \times_{X / G} U, \mathcal{F}\right) \stackrel{\pi_{U, !}}{\longrightarrow} \Gamma_{c}(U, \mathcal{F})
\end{aligned}
$$

où les différentielles sont données par des sommes alternées (dont la forme précise ne nous importe pas) de morphismes associés aux projections $X^{(i)} \rightarrow X^{(i-1)}$. Ce complexe est naturel par rapport aux $X / G$-morphismes $V \stackrel{\phi}{\longrightarrow} U$. En particulier pour $V=X \stackrel{\phi=p}{\longrightarrow} U=X / G$, on obtient un morphisme de complexes

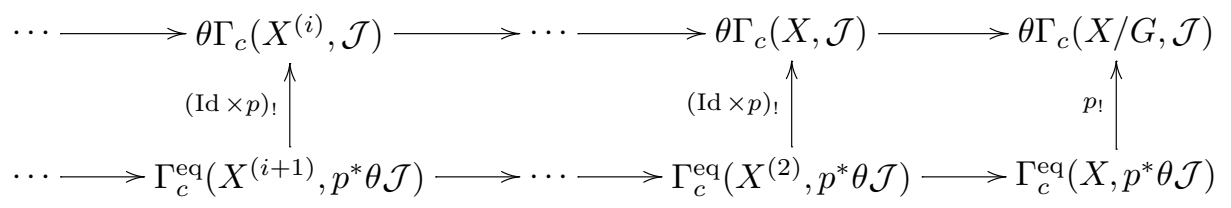

où on a rajouté les symboles eq et $\theta$ pour tenir compte de l'action de $G$. Par hypothèse, le faisceau $\mathcal{J}$ est $\Gamma_{c, \text { loc }}^{\vee}$-acyclique, de sorte que les deux lignes du diagramme ci-dessus sont exactes. D'après la discussion précédente les termes $\Gamma_{c}^{\mathrm{eq}}\left(X^{(i)}, p^{*} \theta \mathcal{J}\right)$ pour $i \geqslant 2$ sont de la forme $\Lambda[G] \otimes_{\Lambda} M$ 
pour un $\Lambda$-module $M$ et sont donc acycliques pour le foncteur $\Lambda \otimes_{\Lambda[G]}-$. On a donc des isomorphismes dans $D^{-}(\Lambda)$ :

$$
\begin{aligned}
\Lambda & \otimes_{\Lambda[G]}^{L} \Gamma_{c}^{\mathrm{eq}}\left(X, p^{*} \theta \mathcal{J}\right) \\
& \simeq\left(\cdots \rightarrow \Lambda \otimes_{\Lambda[G]} \Gamma_{c}^{\mathrm{eq}}\left(X^{(i+1)}, p^{*} \theta \mathcal{J}\right) \rightarrow \cdots \rightarrow \Lambda \otimes_{\Lambda[G]} \Gamma_{c}^{\mathrm{eq}}\left(X^{(2)}, p^{*} \theta \mathcal{J}\right)\right) \\
& \simeq\left(\cdots \rightarrow \Gamma_{c}\left(X^{(i)}, \mathcal{J}\right) \rightarrow \cdots \rightarrow \Gamma_{c}(X, \mathcal{J})\right) \\
& \simeq \Gamma_{c}(X / G, \mathcal{J}) .
\end{aligned}
$$

D'où le lemme, puis la proposition.

Il reste maintenant à prouver le lemme B.3.5.

Cas $\Lambda$ de torsion : dans ce cas, ceci doit être bien connu. Tronquons une résolution injective de $\mathcal{F}$ en degré $2 \operatorname{dim}(X)$ et notons $\mathcal{J} \bullet$ le complexe obtenu. Comme on sait que les foncteurs $\Gamma_{c}(U,-)$ pour $U$ ouvert étale de $X / G$ sont de dimension cohomologique $\leqslant 2 \operatorname{dim}(X)$, les faisceaux $\mathcal{J}^{i}$ sont $\Gamma_{c}$-acycliques au sens donné plus haut. Remarquons maintenant que le complexe de Cech «dual» défini plus haut associé au recouvrement étale $U \stackrel{f}{\longrightarrow} V$ n'est autre que l'image par le foncteur $\Gamma_{c}(V,-)$ du complexe de faisceaux donné par :

$$
\cdots \rightarrow f_{!}^{(i+1)} f^{(i+1)^{*}} \mathcal{J} \stackrel{d_{i+1}}{\longrightarrow} f_{!}^{(i)} f^{(i)^{*}} \mathcal{J} \rightarrow \cdots \stackrel{d_{1}}{\longrightarrow} f_{!} f^{*} \mathcal{J} \stackrel{d_{0}}{\longrightarrow} \mathcal{J}
$$

où $f^{(i)}$ désigne la projection $U \times_{V} \cdots \times_{V} U \rightarrow V$ ( $i$ facteurs) et les différentielles sont des sommes alternées des morphismes induits par les différentes projections. Ce complexe est exact comme on le vérifie aisément sur les fibres. Montrons alors que le complexe de Cech «dual» est lui aussi exact : soit $n \geqslant 0$, tronquons le complexe de faisceaux ci-dessus en degré $-n-2 \operatorname{dim}(X)-1$ :

$$
C_{-n-2 \operatorname{dim}(X)-1} \rightarrow f_{!}^{(n+2 \operatorname{dim}(X))} f^{(n+2 \operatorname{dim}(X))^{*}} \mathcal{J} \rightarrow \cdots \rightarrow \mathcal{J},
$$

on obtient une résolution du premier terme $C_{-n-2 \operatorname{dim}(X)-1}$ par des faisceaux $\Gamma_{c}$-acycliques. En appliquant le foncteur $\Gamma_{c}(V,-)$ on obtient un complexe dont la cohomologie est

$$
\mathcal{H}^{q}=H_{c}^{n+2 \operatorname{dim}(X)+1+q}\left(V, C_{-n-2 \operatorname{dim}(X)}\right)
$$

qui est nul pour $q \geqslant-n$. Ceci montre l'exactitude du complexe de Cech «dual» en degré $-n$.

Cas $\Lambda$ anneau l-adique : Soit $\left(\mathcal{F}_{n}\right)_{n} \in \operatorname{Mod}_{\Lambda} .\left(X / G_{\mathrm{et}}\right)$. Comme auparavant, tronquons une résolution de $\left(\mathcal{F}_{n}\right)_{n}$ par des objets injectifs de $\operatorname{Mod}_{\Lambda} .\left(X / G_{\text {et }}\right)$ en degré $2 \operatorname{dim}(X)+1$. Le complexe obtenu $\left(\mathcal{J}_{n}\right)_{n}^{\bullet}$ est formé de $\Lambda_{\bullet}$-faisceaux $\Gamma_{c}$-acycliques pour la même raison que dans le cas de torsion. On sait par ailleurs que le foncteur $\varliminf^{X / G}$ est aussi de dimension cohomologique $\leqslant 2 \operatorname{dim}(X)+1$. Une façon de le voir est la suivante : en raisonnant sur les fibres on voit que

$$
\operatorname{dim} . \operatorname{coh} .\left(\varliminf^{X / G}\right) \leqslant \sup _{U \rightarrow X / G} \operatorname{dim} . \operatorname{coh} .\left(\Gamma(U,-) \circ \varliminf^{U}\right)
$$

et pour tout $U \rightarrow X / G$, on a $R\left(\Gamma(U,-) \circ \varliminf^{U}\right)=R\left(\varliminf_{\lim } \circ \Gamma(U,-)\right)=R \varliminf \circ R \Gamma(U,-)$ où l'on sait que $\varliminf_{i m}: \operatorname{Mod}\left(\Lambda_{\bullet}\right) \rightarrow \operatorname{Mod}(\Lambda)$ est de dimension cohomologique $\leqslant 1$ et que $\Gamma(U,-)$ est de dimension cohomologique $2 \operatorname{dim}(X)$.

Ainsi les $\Lambda_{\bullet}$-faisceaux $\left(\mathcal{J}_{n}\right)_{n}^{i}$ sont aussi lim-acycliques, et par conséquent les $\Lambda$-faisceaux $\left(\varliminf_{\mathcal{J}} \mathcal{J}_{n}\right)^{i}$ sont $\Gamma_{!}(U,-)$-acycliques pour tout morphisme étale $U \rightarrow X$, puisqu'on a la 
factorisation $R \Gamma_{c}(U,-)=R \Gamma_{!}(U,-) \circ R \gtreqless$. On aimerait alors conclure par le même raisonnement que dans le cas de torsion que les $\Lambda$-faisceaux $\left(\varliminf_{j} \mathcal{J}_{n}\right)^{i}$ sont $\Gamma_{!, \text {et }}^{\vee}$-acycliques (ce qui est équivalent à dire que les $\Lambda_{\bullet}$-faisceaux $\left(\mathcal{J}_{n}\right)_{n}^{i}$ sont $\Gamma_{c \text {,et }}^{\vee}$-acycliques). Mais il nous manque la finitude cohomologique des foncteurs $\Gamma_{!}(V,-)$ sur les catégories de $\Lambda$-faisceaux ( $\Lambda$ n'est pas de torsion).

L'auteur ne sait pas si l'assertion du lemme est vraie pour les revêtements $U \stackrel{f}{\rightarrow} V$ étales quelconques. Mais dans le cas où $f$ est un isomorphisme local, on peut procéder de la manière suivante. Introduisons le site $V_{\text {loc }}$ dont les objets sont les isomorphismes locaux $W \rightarrow V$, avec la définition habituelle d'un recouvrement. On a des morphismes de sites évidents $V_{\text {et }} \stackrel{\rho^{V}}{\longrightarrow}$ $V_{\text {loc }} \stackrel{\mu^{V}}{\longrightarrow}|V|$ où $|V|$ est le site topologique de $V$. Les foncteurs $\mu_{*}^{V}$ et $\mu^{V^{*}}$ induisent des équivalences réciproques de topos. Il s'ensuit que le foncteur $\Gamma_{!}^{\operatorname{loc}}(V,-)$ des sections à support compact $\operatorname{sur} \operatorname{Mod}_{\Lambda}\left(\widetilde{V_{\text {loc }}}\right)$ est de dimension cohomologique $\leqslant \operatorname{dim}(V)=\operatorname{dim}(X)$, et ceci quel que soit l'anneau $\Lambda$.

On a une factorisation $\Gamma_{c}(V,-)=\Gamma_{!}^{\operatorname{loc}}(V,-) \circ \rho_{*}^{V} \circ \varliminf^{V_{\text {et }}}$. Montrons maintenant que le foncteur exact à gauche $\rho_{*}^{V} \circ \varliminf^{V_{\mathrm{et}}}: \operatorname{Mod}_{\Lambda}\left(\widetilde{V_{\mathrm{et}}}\right) \rightarrow \operatorname{Mod}_{\Lambda}\left(\widetilde{V_{\mathrm{loc}}}\right)$ est de dimension cohomologique

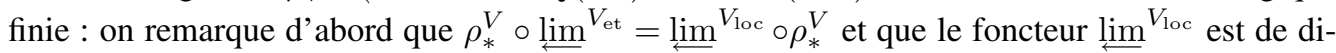
mension cohomologique $\leqslant \operatorname{dim}(V)+\overleftarrow{1}=\operatorname{dim}(X)+1$ pour la même raison que plus haut. En dérivant on obtient

$$
R\left(\rho_{*}^{V} \circ \varliminf^{V_{\mathrm{et}}}\right)=R\left(\varliminf^{V_{\mathrm{loc}}} \circ \rho_{*}^{V}\right)=R \varliminf^{V_{\mathrm{loc}} \circ R \rho_{*}^{V},}
$$

la seconde égalité venant de ce que $\rho_{*}^{V}$ envoie injectifs sur injectifs puisqu'il a un adjoint à gauche exact. Maintenant pour tout faisceau abélien étale $\mathcal{F}$, et tout $x \in V$ la fibre de $R^{q} \rho_{*}^{V}(\mathcal{F})$ en $x$ est donnée comme dans [2, 4.2.4] (et avec les notations de loc. cit.) par

$$
R^{q} \rho_{*}^{V}(\mathcal{F})_{x} \simeq H^{q}\left(\operatorname{Gal}\left(\mathcal{H}(x)^{\mathrm{ca}} / \mathcal{H}(x)\right), \mathcal{F}_{x}\right)
$$

D'après $[2,2.5 .1]$ et notre hypothèse $K$ algébriquement clos, on en déduit que $\rho_{*}^{V}$ est de dimension cohomologique $\leqslant \operatorname{dim}(X)$ sur la catégorie $\operatorname{Mod}_{\Lambda}$. $\left(\widetilde{V_{\text {et }}}\right)$. On a donc obtenu que le foncteur $\rho_{*}^{V} \circ \varliminf^{V \text { et }}$ est de dimension cohomologique $\leqslant 2 \operatorname{dim}(X)+1$. Il s'ensuit que les $\Lambda_{\bullet}$-faisceaux $\left(\mathcal{J}_{n}\right)_{n}^{i}$ sont acycliques pour ce foncteur et par conséquent que les $\Lambda$-faisceaux $\rho_{*}^{V} \circ \varliminf^{V_{\text {et }}}\left(\mathcal{J}_{n}\right)^{i}$ sur $V_{\text {loc }}$ sont $\Gamma_{!}^{\text {loc }}(V,-)$-acycliques !

À partir de là, on prouve par un raisonnement similaire au cas de torsion que les faisceaux $\rho_{*}^{V} \circ \varliminf^{V_{\text {et }}}\left(\mathcal{J}_{n}\right)^{i}$ sur $V_{\text {loc }}$ sont $\Gamma_{! ! \text {loc }}^{\vee}$-acycliques, ce qui est encore équivalent à dire que les $\Lambda_{\bullet}$-faisceaux $\left(\mathcal{J}_{n}\right)_{n}^{i}$ sont $\Gamma_{c, \text { loc }}^{\vee}$-acycliques au sens du lemme.

B.3.7. Corollaire. - Soit $\mathcal{F}$ un $\Lambda$-faisceau étale sur $X / G$ si $\Lambda$ est de torsion ou un $\Lambda$-système local si $\Lambda$ est l-adique. Il existe une suite spectrale

$$
E_{2}^{p q}=\operatorname{Tor}_{p}^{G}\left(\Lambda, H_{c}^{q}\left(X, p^{*} \mathcal{F}\right)\right) \Longrightarrow H_{c}^{q-p}(X / G, \mathcal{F})
$$

Remarquons que lorsque $\Lambda$ est un corps ou un anneau Gorenstein, ou $\mathcal{F}$ est $l$-adique, on obtient en dualisant la suite spectrale $[19,4.4 .1]$.

\section{RÉFÉRENCES}

[1] Bernstein J., Beilinson A., Deligne P., Analyse et topologie sur les espaces singuliers (I : Faisceaux Pervers), Astérisque, 100.

$4^{\text {e }}$ SÉRIE - TOME $39-2006-\mathrm{N}^{\circ} 1$ 
[2] Berkovich V.G., Étale cohomology for non-archimedean analytic spaces, Publ. Math. IHÉS 78 (1993) 1-159.

[3] BERKOVICH V.G., Vanishing cycles for formal schemes, Invent. Math. 115 (1994) 539-571.

[4] Berkovich V.G., The automorphism group of the Drinfeld half-space, C. R. Acad. Sci. Paris 321 (1995) 1127-1132.

[5] BERKOVICH V.G., Etale equivariant sheaves on $p$-adic analytic spaces, 1995.

[6] BERKOVICH V.G., On the comparison theorem for etale cohomology of non-archimedean analytic spaces, Israel J. Math. 92 (1995) 45-60.

[7] Berkovich V.G., Vanishing cycles for formal schemes II, Invent. Math. 125 (1996) 367-390.

[8] Bernstein I.N., Zelevinski A.V., Induced representations of reductive p-adic groups, Ann. Sci. École Norm. Sup. 10 (1977) 441-472.

[9] Bernstein J., Le centre de Bernstein, in : Bernstein J., Deligne P., Kazhdan D., Vignéras M.F. (Eds.), Représentations des groupes réductifs sur un corps local, in: Travaux en cours, Hermann, Paris, 1984.

[10] Boutot J.-F., Carayol H., Uniformisation $p$-adique des courbes de Shimura : les théorèmes de Cerednik et Drinfeld, in: Courbes modulaires et courbes de Shimura, in : Astérisque, vols. 196197, SMF, Paris, 1991, pp. 45-158.

[11] BRown K.S., Cohomology of Groups, Graduate Texts Math., vol. 87, Springer, Berlin, 1982.

[12] BRUhat F., Tits J., Groupes réductifs sur un corps local I, vol. 41, IHÉS, 1972.

[13] Carayol J.S., Non-abelian Lubin-Tate theory, in : Clozel L., Milne J.S. (Eds.), Automorphic Forms, Shimura Varieties and L-Functions, vol. II, Academic Press, New York, 1990, pp. 15-39.

[14] DAT J.-F., $\nu$-tempered representations of $p$-adic groups I: $l$-adic case, Duke Math. J. 126 (3) (2005) 397-469.

[15] Deligne P., Théorèmes de Lefschetz et critères de dégénérescence de suites spectrales, Publ. Math. IHÉS 35 (1968) 259-278.

[16] Deligne P., Les constantes des équations fonctionnelles des fonctions $L$, in : Modular Functions of One Variable II, in : Lecture Notes in Math., vol. 349, Springer, Berlin, 1972, pp. 501-597.

[17] DRINFELD V.G., Elliptic modules, Math. USSR Sb. 23 (1974) 561-592.

[18] Faltings G., A relation between two moduli spaces studied by Drinfeld, in: Algebraic Number Theory and Algebraic Geometry, in : Contemp. Math., vol. 300, 2002, pp. 115-129.

[19] Fargues L., Cohomologie d'espaces de modules de groupes $p$-divisibles et correspondances de Langlands locale, in: Astérisque, vol. 291, SMF, Paris, 2003.

[20] HARRIS M., Supercuspidal representations in the cohomology of Drinfeld's upper half space; elaboration of Carayol's program, Invent. Math. 129 (1997) 75-119.

[21] HARRIS M., Cohomological automorphic forms on GL(n), J. Math. Kyoto Univ. 39 (1999) 299-318.

[22] HARris M., TAYlor R., The Geometry and Cohomology of Some Simple Shimura Varieties, in: Ann. of Math. Stud., vol. 151, Princeton University Press, Princeton, NJ, 2001.

[23] Hausberger T., Uniformisation des variétés de Laumon-Rapoport-Stuhler et preuve de la conjecture de Drinfeld-Carayol, Ann. Inst. Fourier 55 (4) (2005) 1285-1371.

[24] Henniart G., Une preuve simple des conjectures de Langlands pour $G L(n)$ sur un corps $p$-adique, Invent. Math. 139 (2000) 439-455.

[25] Henniart G., Sur la conjecture de Langlands locale pour $G L(n)$, J. Théorie Nombres Bordeaux 13 (2001) 167-187.

[26] Henniart G., Une caractérisation de la correspondance de Langlands locale pour GL(n), Bull. Soc. math. France 130 (4) (2002) 587-602.

[27] Huber R., A comparison theorem for l-adic cohomology, Compositio Math. 112 (1998) 217-235.

[28] Illusie L., Autour du théorème de monodromie locale, in: Périodes p-adiques, in: Astérisque, vol. 223, SMF, Paris, 1994, pp. 9-57.

[29] Iто T., Weight-monodromy conjecture for $p$-adically uniformized varieties, Invent. Math. 159 (3) (2005) 607-656.

[30] JANnsen U., Continuous étale cohomology, Math. Ann. 280 (1988) 207-245.

[31] LAUMon G., RApoport M., StUhler U., D-elliptic sheaves and the Langlands correspondence, Invent. Math. 113 (1993) 217-338. 
[32] Mustafin G.A., Nonarchimedean uniformization, Math. USSR Sb. 34 (1978) 187-214.

[33] ORLIK S., The cohomology of period domains for reductive groups over local fields, Preprint, University Leipzig, 2002.

[34] ORLiK S., On extensions of generalized Steinberg representations, J. Algebra 293 (2) (2005) 611-630.

[35] RAPOPORT M., ZINK T., Über die lokale Zetafunktion von Shimuravarietäten, Monodromiefiltration und verschwindende Zyklen in ungleicher Characteristik, Invent. Math. 68 (1982) 21-101.

[36] RoDIER F., Décomposition de la série principale d'un groupe réductif $p$-adique ? in : Lecture Notes in Math., vol. 880, Springer, Berlin, 1981, pp. 408-424.

[37] SaIto T., Weight spectral sequences and independence of $l$, J. Institut Math. Jussieu 1 (4) (2002).

[38] Schneider P., Stuhler U., The cohomology of $p$-adic symmetric spaces, Invent. Math. 105 (1991) $47-122$.

[39] SChNeIder P., STUhler U., Resolutions for smooth representations of $p$-adic $G L(n)$, J. reine angew. Math. 436 (1993) 19-32.

[40] Schneider P., Stuhler U., Representation theory and sheaves on the Bruhat-Tits building, Publ. Math. IHÉS 85 (1995) 97-191.

[41] SGA5, Cohomologie $l$-adique et fonctions $L$, in : L. Illusie (Ed.), Séminaire de Géometrie Algébrique du Bois-Marie 1865-1966 (SGA5), in : Lecture Notes in Math., vol. 589, Springer, Berlin, 1977, pp. xii +484 .

[42] Vignéras M.-F., Représentations modulaires de $G L(2, F)$ en caractéristique $l, F$ corps $p$-adique, $p \neq l$, Composito Math. 72 (1989) 33-66.

[43] Vignéras M.-F., Cohomology of sheaves on the building, Invent. Math. 127 (1997) 349-373.

[44] VignÉRAS M.-F., Modular representations of $p$-adic groups, ICM III (2002) 667-677.

[45] VignéRAS M.-F., Représentations $l$-modulaires d'un groupe $p$-adique avec $l$ différent de $p$, in: Progress in Math., vol. 137, Birkhäuser, Basel, 1996.

\author{
Jean François DAT \\ Université Paris XIII, \\ LAGA - UMR 7539, \\ Institut Galilée (Mathématiques), \\ Avenue J.-B. Clément, \\ Villetaneuse 93430, France \\ E-mail : dat@math.univ-paris13.fr
}

$4^{\mathrm{e}}$ SÉRIE - TOME $39-2006-\mathrm{N}^{\circ} 1$ 